\title{
Immediate and delayed responses of power lines and transformers in the Czech electric power grid to geomagnetic storms
}

\author{
Michal Švanda ${ }^{1,2, *}$, Didier Mourenas ${ }^{3}$, Karla Žertová $^{4}$, and Tatiana Výbošt'oková ${ }^{5}$ \\ ${ }_{1}^{1}$ Astronomical Institute of the Czech Academy of Sciences, 25165 Ondřejov, Czech Republic \\ 2 Astronomical Institute, Charles University, 18000 Praha, Czech Republic \\ ${ }^{3}$ CEA, DAM, DIF, 91297 Arpajon, France \\ ${ }^{4}$ Gymnázium Jiř́ho Ortena, 28480 Kutná Hora, Czech Republic \\ 5 Department of Surface and Plasma Science, Charles University, 18000 Praha, Czech Republic
}

Received 24 February 2020 / Accepted 29 May 2020

\begin{abstract}
Eruptive events of solar activity often trigger abrupt variations of the geomagnetic field. Through the induction of electric currents, human infrastructures are also affected, namely the equipment of electric power transmission networks. It was shown in past studies that the rate of power-grid anomalies may increase after an exposure to strong geomagnetically induced currents. We search for a rapid response of devices in the Czech electric distribution grid to disturbed days of high geomagnetic activity. Such disturbed days are described either by the cumulative storm-time $D s t$ or d(SYM-H)/d $t$ low-latitude indices mainly influenced by ring current variations, by the cumulative $A E$ high-latitude index measuring substorm-related auroral current variations, or by the cumulative ap mid-latitude index measuring both ring and auroral current variations. We use superposed epoch analysis to identify possible increases of anomaly rates during and after such disturbed days. We show that in the case of abundant series of anomalies on power lines, the anomaly rate increases significantly immediately (within 1 day) after the onset of geomagnetic storms. In the case of transformers, the increase of the anomaly rate is generally delayed by 2-3 days. We also find that transformers and some electric substations seem to be sensitive to a prolonged exposure to substorms, with a delayed increase of anomalies. Overall, we show that in the 5-day period following the commencement of geomagnetic activity there is an approximately 5-10\% increase in the recorded anomalies in the Czech power grid and thus this fraction of anomalies is probably related to an exposure to GICs.
\end{abstract}

Keywords: Spaceweather / Geomagnetically induced currents / Impacts on technological systems

\section{Introduction}

The Sun is a magnetically active star, filling the interplanetary space with a stream of charged particles called the solar wind (see e.g. a recent review by Verscharen et al., 2019). The solar wind properties are far from being homogeneous, with strong variations in temperature, density, or interplanetary magnetic field observed in connection with various phenomena of solar activity. The main drivers of strong disturbances of the solar wind are coronal mass ejections (CMEs), the fast-slow solar wind interaction on the borders of corotating interaction regions, and fast-wind outflows from coronal holes. Solar-wind disturbances may ultimately interact with Earth's magnetosphere, thereby triggering geomagnetic activity.

As first proposed by Dungey (1961), the dynamic pressure exerted by the solar wind on the magnetosphere can trigger

\footnotetext{
*Corresponding author: mi chal@astronomie.cz
}

magnetic reconnection, opening dayside dipolar geomagnetic field lines. The solar wind then transports this magnetic field to the nightside, forming a long tail behind the Earth. This transfer of magnetic flux and the resulting reconfiguration of the magnetosphere eventually leads to nightside magnetic reconnection, returning flux to the dayside in various phenomenological response modes that depend on the disturbance level (Dungey, 1961; Kepko et al., 2014). However, a common characteristic of all such response modes is the formation of a current wedge system (Kepko et al., 2014; McPherron \& Chu, 2017). A fraction of the tail current along geomagnetic field lines is then temporarily diverted through the ionosphere, allowing a closure of the current wedge and causing perturbations in the auroral zone and at middle latitudes (McPherron \& Chu, 2017).

Both substorms and geomagnetic storms give rise to a current wedge, plasma sheet inward convection by inductive electric fields, and energetic particle injections (Thomsen, 2004; Kepko et al., 2014; Ganushkina et al., 2017; McPherron \& Chu, 2017). 
However, the current wedge has generally a more limited temporal extent during substorms than during storms, which frequently last for days (Kepko et al., 2014; Ganushkina et al., 2017). Substorms are one of the key dynamical processes occurring during storms, but isolated substorms also occur outside storms (Viljanen et al., 2006; Turnbull et al., 2009). During storms (mainly caused by strong interactions between CMEs and the magnetosphere), a stronger buildup of the inner ring current (a westward current of ions roughly 2-4 Earth radii above the equator) is provided by a deeper inward transport of charged particles from the plasma sheet, leading to a significant and prolonged decrease of the geomagnetic field (Ganushkina et al., 2017).

All these ionospheric and magnetospheric currents, and the related field-aligned currents, can cause important geomagnetic field variations during periods of rapidly evolving solar wind dynamic pressure (Gonzalez et al., 1994; Kappenman, 2005; Tsurutani et al., 2009; Lakhina \& Tsurutani, 2016; McPherron $\&$ Chu, 2017). This realization has led to the traditional concept of disturbed days: days of smooth and regular geomagnetic field variations have been called quiet days, whereas days of stronger and irregular variations have been called disturbed days (Chapman \& Bartels, 1940).

Geomagnetically induced currents (GICs) in the ground are due to strong variations $\mathrm{d} H / \mathrm{d} t$ of the horizontal component $H$ of the geomagnetic field over typical time scales of $\sim 10-1000 \mathrm{~s}$ during disturbed days (Kappenman, 2003; Kataoka \& Pulkkinen, 2008; Carter et al., 2015; Zhang et al., 2016; Pokhrel et al., 2018). Substorms generally produce the largest $\mathrm{d} H / \mathrm{d} t$ at high and mid-latitudes during periods of fast solar wind and have caused many of the major GIC impacts during large storms - e.g., the Quebec voltage collapse on 13 March 1989 was triggered by a first substorm, while two later substorms tripped out transformers in the UK (Boteler, 2019). $\mathrm{d} H / \mathrm{d} t$ was found to be twice smaller in general during non-storm substorms than during storm-related substorms, possibly due to an additional input from ring current variations during storms (Viljanen et al., 2006; Turnbull et al., 2009). Other important sources of $\mathrm{d} H / \mathrm{d} t$ during geomagnetic storms include sudden commencements (the shock compression of the magnetosphere when a fast CME impacts the magnetosphere at the start of a storm, leading to an increase of Chapman-Ferraro currents at the dayside magnetopause; e.g., see Kikuchi et al., 2001) and rapid variations of the ring current, through its role in the generation of Region 2 field-aligned currents (Ganushkina et al., 2017). Sudden commencements have a large $\mathrm{d} H / \mathrm{d} t$ because of their shock-like nature, while rapid increases of ring current energy density following large scale injection or inward convection of energetic charged particles coming from the plasma sheet can also produce large $\mathrm{d} H / \mathrm{d} t$ (Kappenman, 2003, 2005; Kataoka \& Pulkkinen, 2008).

GICs propagate through conducting regions in the ground and water, but also in the grounded conductors. The presence of GICs in the electric power grid can cause various kinds of damage. GICs are quasi-DC currents that can lead to half-cycle saturation and drive a transformer response into a non-linear regime. This poses a risk for transformers by producing high pulses of magnetizing current, a local heating (also vibration) within the transformer (Gaunt, 2014), and the generation of $\mathrm{AC}$ harmonics that propagate out into the power network, where they can disrupt the operations of various devices (Molinski,
2002; Kappenman, 2007). In particular, the propagation of harmonics in the power grid during half-cycle saturation can distort the electrical current waveform, eventually triggering a detrimental reaction of protective relays connecting power lines, or leading to a disruption of other devices attached to these lines.

GICs identified by fast variations of the geomagnetic field have been linked with various power grid failures (Schrijver \& Mitchell, 2013), eventually leading to power grid disruptions (Pirjola, 2000; Kappenman, 2007; Schrijver \& Mitchell, 2013; Pulkkinen et al., 2017). Although high latitude regions are more at risk from GICs, middle and low latitude regions may also be impacted by significant GICs (Gaunt \& Coetzee, 2007; Watari et al., 2009; Marshall et al., 2012; Torta et al., 2012; Zois, 2013; Carter et al., 2015; Wang et al., 2015; Zhang et al., 2016; Bailey et al., 2017; Lotz \& Danskin, 2017; Tozzi et al., 2019).

A first study of anomalies in the Czech power grid as a function of geomagnetic activity (defined by the $K$ index computed from the measurements of the Earth's magnetic field at a local magnetometer station near Budkov - e.g., see Mayaud, 1980; McPherron \& Chu, 2017) has already identified some statistically significant increases of the rate of anomalies around month-long periods of higher geomagnetic activity than nearby periods of lower activity (Výbošt'oková \& Švanda, 2019). Nevertheless, the relationship between geomagnetic events and anomalies still remained somewhat loose.

Accordingly, the main goal of the present paper is to better ascertain the existence of a tight relationship between power grid anomalies and geomagnetic storms, on the basis of the same data set. We shall discuss the physical mechanisms by which GICs may cause anomalies in power lines and transformers, and show that our statistical results are suggestive of a causal relationship based on those mechanisms. We shall also address the important and unanswered question of the time delay between moderate to large geomagnetic storms with minimum Dst < -40 nT (Gonzalez et al., 1994) and the actual occurrences of anomalies. For that purpose, we shall use Superposed Epoch Analysis to investigate the relative occurrence of GIC effects in the Czech power grid during disturbed days as compared with quiet days. Such disturbed days will be categorized using different time-integrated parameters of geomagnetic activity, related to the magnitude of temporal variations of the horizontal component of the geomagnetic field, which can induce detrimental currents in power lines.

\section{Data sets}

In this study, we searched for a causal relation between two types of time series. The first series describing the daily anomaly rates in the Czech electric power-distribution grid, and the second serving as a proxy of disturbed days for the estimation of geomagnetically induced currents.

\subsection{Logs of anomalies}

The Czech Republic is a mid-latitude country (around $\sim 50^{\circ}$ geographic latitude and $\sim 45^{\circ}$ corrected geomagnetic latitude), where the effects of solar/geomagnetic activity on ground-based infrastructures is expected to be moderate at most. 
Table 1. Datasets analysed in this study. This is a reduced version of Table 1 in Výbošt'oková \& Švanda (2019).

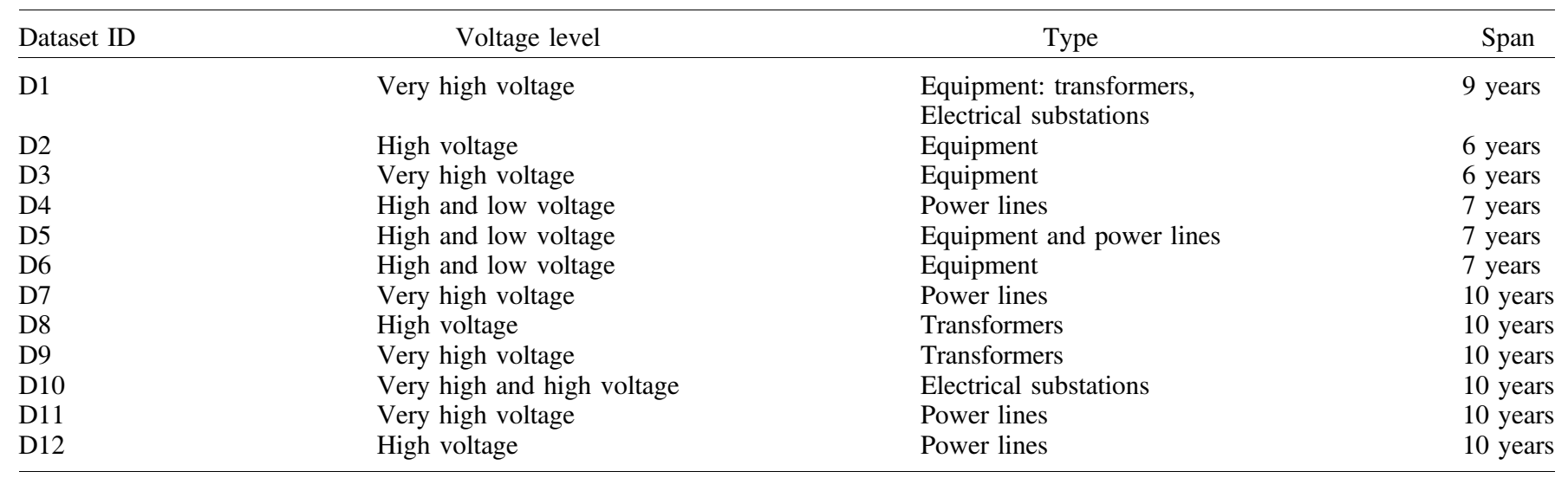

The modelled amplitudes of GICs during the Halloween storms in late October 2003 reached 1-minute peaks of about 60 A. ${ }^{1}$ The country has a shape prolonged in the east-west direction (about $500 \mathrm{~km}$ length), whereas in the south-north direction it is about $280 \mathrm{~km}$ long from border to border. The spine of the electric power network is operated by the national operator ČEPS, a.s., which maintains the very-high-voltage $(400 \mathrm{kV}$ and $220 \mathrm{kV}$ ) transmission network, and connects the Czech Republic with neighbouring countries. ČEPS also maintains the key transformers and electrical substations in the transmission network. The area of the state is then split into three regions, where the electricity distribution is under the responsibility of the distribution operators. The southern part is maintained by E.ON Distribuce, a.s., the northern part by ČEZ Distribuce, a.s., and the capital city of Prague is maintained by PREdistribuce, a.s. All three distributors maintain not only very-high-voltage $(110 \mathrm{kV})$ and high-voltage $(22 \mathrm{kV})$ power lines, but also connect the consumers via the low-voltage $(400 \mathrm{~V})$ electric power transmission network.

All four above-mentioned power companies have agreed to provide us their maintenance logs. The datasets used in this study are exactly the same datasets already used in the study by Výbošt'oková \& Švanda (2019). Thus, we refer the reader to Section 3.2 of this previous paper for a more detailed description of the datasets. By mutual non-disclosure agreement with the data providers, the datasets were anonymised (by removing the information about the power-company name, and also by changing the calendar date to a day number) and must be presented as such. The total time span is 12 years, but the span of individual maintenance logs provided by the operators is shorter, varying between 6 and 10 years.

We only briefly recall that the obtained logs were cleaned from events that were obviously not related to variations of geomagnetic activity. From these logs, we keep only the dates when the events occurred and did not consider any other details. These inhomogeneous datasets (the log entries were provided by different individuals with varying levels of details and quality of the event description) were split into 12 subsets D1-D12,

\footnotetext{
${ }^{1}$ Smičková, A., Geomagnetically Induced Currents in the Czech Power Grid, BSc. thesis (supervisor Švanda, M.), Faculty of Electrical Engineering, Czech Technical University, 2019, available online http://hdl.handle.net/10467/84988.
}

which were investigated separately. Each sub-dataset was selected so that it contained only events occurring on devices of a similar type and/or with the same voltage level and were recorded by the same operating company. The dataset descriptions are briefly summarised in Table 1.

\subsection{Geomagnetic indices and parameters used for GIC estimation}

Various parameters have been considered to estimate the effects of geomagnetic activity on power grids (Schrijver \& Mitchell, 2013). GICs are due to strong variations $\mathrm{d} H / \mathrm{d} t$ over typical time scales of $\sim 10-1000 \mathrm{~s}$ (Kappenman, 2003). There are two sources of such large $\mathrm{d} H / \mathrm{d} t$ at low and middle latitudes: (i) sudden impulses (SI), also called sudden commencements (SC) when they are followed by a storm caused by the shock preceding a fast $\mathrm{CME}$, and (ii) the growth and decay of the ring current during a magnetic storm. Substorm-related disturbances are mostly limited to high and middle latitudes, whereas disturbances caused by ring current changes generally affect mainly middle and low latitudes. Statistically, periods of stronger cumulative effects of GICs in a power grid are therefore expected to correspond to disturbed days of elevated geomagnetic activity (Chapman \& Bartels, 1940). In the present study, we shall use various cumulative (time-integrated) parameters based on different magnetic indices to categorize such disturbed days, and we shall investigate the relative occurrence of GIC effects during such disturbed days as compared with quiet days.

An appropriate quantity to estimate GICs at low latitudes is $\mathrm{d}(\mathrm{SYM}-\mathrm{H}) / \mathrm{d} t$, which directly provides a (longitudinally averaged) measure of the 1 -minute $\mathrm{d} H / \mathrm{d} t$ due to ring current variations that drive GICs there (Kappenman, 2003; Carter et al., 2015; Zhang et al., 2016). Indeed, the SYM-H index is essentially similar to the hourly $D s t$ storm time index, but measured on 1-minute time scales - that is, it provides the disturbance $\Delta H=H-H_{\text {quiet }}$ of the horizontal component of the magnetic field as compared to its quiet-time level, longitudinally averaged based on ground magnetometer measurements at different low latitude magnetometer stations (Mayaud, 1980).

Several studies have demonstrated the existence of significant correlations between GICs or electric grid failures and times of large $\mathrm{d}(\mathrm{SYM}-\mathrm{H}) / \mathrm{d} t$ at low to middle latitudes during geomagnetic storms, although $\mathrm{d}(\mathrm{SYM}-\mathrm{H}) / \mathrm{d} t$ is often 
inappropriate during strong substorms (Carter et al., 2015; Wang et al., 2015; Zhang et al., 2016). Carter et al. (2015) have further shown that the actual $\mathrm{d} H / \mathrm{d} t$ at middle latitudes due to SI/SCs can be a factor $\sim 2-3$ larger on the dayside than $\mathrm{d}(\mathrm{SYM}-\mathrm{H}) / \mathrm{d} t$, potentially allowing GIC effects even during geomagnetic events with relatively small $\mathrm{d}(\mathrm{SYM}-\mathrm{H}) / \mathrm{d} t$. We checked that $\mathrm{d} H / \mathrm{d} t$ at the Czech magnetometer station of Budkov can also be sometimes $>2-3$ times larger than $\mathrm{d}(\mathrm{SYM}-\mathrm{H}) / \mathrm{d} t$ during SI/SCs. Viljanen et al. (2014) have noticed the presence of a European region of low underground conductivity stretching from France through Czech Republic to Hungary that could favor significant GICs at middle latitudes. Gil et al. (2019) have shown the presence of GICs during a few selected storms in Poland, while Tozzi et al. (2019) have found that non-negligible GICs could exist even down to northern Italy. Wang et al. (2015) have further emphasized that cumulative GICs in a nuclear plant transformer during a longduration geomagnetic event could sometimes be more harmful than short events, due to the longer cumulated time of transformer heating.

Accordingly, we consider here the $\operatorname{Int}(\mathrm{d}(\mathrm{SYM}-\mathrm{H}) / \mathrm{d} t)$ parameter to categorize disturbed days of expected significant GIC impacts on power grids. $\operatorname{Int}(\mathrm{d}(\mathrm{SYM}-\mathrm{H}) / \mathrm{d} t)$ is calculated over each day, as the sum of all 1-minute $|\mathrm{d}(\mathrm{SYM}-\mathrm{H}) / \mathrm{d} t|$ values (in $\mathrm{nT} / \mathrm{min}$ ) obtained during times when SYM-H remains smaller than some threshold. The selected threshold (varying from $-50 \mathrm{nT}$ to $-25 \mathrm{nT}$ ) should ensure that only geomagnetic storm periods are considered (Gonzalez et al., 1994). This $\operatorname{Int}(\mathrm{d}(\mathrm{SYM}-\mathrm{H}) / \mathrm{d} t)$ parameter allows, in principle, to take into account the immediate effects on power grids caused by large individual $|\mathrm{d} H / \mathrm{d} t|$ due to ring current variations, as well as the more delayed, cumulative effects potentially caused by prolonged periods of moderate to significant $|\mathrm{d} H / \mathrm{d} t|$ levels (Carter et al., 2015; Wang et al., 2015; Zhang et al., 2016) - although large individual $|\mathrm{d} H / \mathrm{d} t|$ during strong substorms will need other indices such as $A E$ or ap to take them into account (see below).

Other works have suggested that the mean or cumulative Dst during storm main phase should be good indicators of long duration GICs, because larger and steeper decreases of Dst correspond to stronger disturbances that should generally lead to larger $\mathrm{d} H / \mathrm{d} t$ at the relevant shorter time scales of $\sim 10-1000 \mathrm{~s}$ (Balan et al., 2014, 2016; Lotz \& Danskin, 2017). Using observations in South Africa (at middle corrected geomagnetic latitudes $\sim 36^{\circ}-42^{\circ}$ not much lower than in the Czech Republic), Lotz \& Danskin (2017) have demonstrated the existence of a linear relationship between the sum of induced electric fields recorded in the ground during geomagnetic storms and the integral of SYM-H (or Dst) values, suggesting that the cumulative SYM-H or Dst could be used as good proxies for cumulated induced electric fields at middle corrected geomagnetic latitudes (although ring current effects are likely more important for GICs in South Africa than in the Czech Republic, where a more balanced mixture of ring current and substorm effects is present). They also noted that some effects might be present as long as SYM-H remained below $-20 \mathrm{nT}$.

Therefore, we also consider the IntDst parameter to categorize disturbed days of expected significant GICs in the Czech Republic (e.g., see Mourenas et al., 2018). IntDst (in nT hr) is calculated as a sum of hourly $|D s t|$ values. This summation starts when Dst first becomes smaller than a threshold (taken between $-50 \mathrm{nT}$ and $-25 \mathrm{nT}$ as before) chosen to ensure that only storm periods are considered, and this summation ends when Dst reaches its minimum value over the next $24 \mathrm{~h}$. Each IntDst value is then assigned to the starting day of a given summation, with all integration periods strictly separated by construction. As a result, IntDst is generally measured during storm main phase, where the effects on GICs are likely stronger (Balan et al., 2014, 2016), to provide a complementary metric to the Int $(\mathrm{d}(\mathrm{SYM}-\mathrm{H}) / \mathrm{d} t)$ metric calculated over each whole day without any consideration of storm phase.

While ring current variations during storms can be quantified by Dst and SYM-H indices, the magnetic indices that provide a measure of magnetospheric and ionospheric current variations observed during strong substorms are $A E, A L, K p$, or ap (Mayaud, 1980; Kamide \& Kokobun, 1996; Thomsen, 2004; Mourenas et al., 2020). The ap index (as its logarithmic equivalent $K p$ ) provides a global measure of the range of magnetic field variations at middle latitudes over 3-h time scales, obtained by averaging measurements from different midlatitude magnetometer stations spread in longitude (Mayaud, 1980; Thomsen, 2004). In contrast, the range indices $A E$ and $A L$ are measured at higher magnetic latitudes $>60^{\circ}$ inside the auroral region (Mayaud, 1980; Kamide \& Rostoker, 2004), and $A E$ saturates at high geomagnetic activity am $>150$ (with am a mid-latitude index similar to $a p$ ) because the auroral oval then expands equatorward of the magnetometer stations measuring it (Thomsen, 2004; Lockwood et al., 2019). Therefore, $a p$ is probably more appropriate than $A E$ for quantifying the strength of time-integrated geomagnetic disturbances at middle (sub-auroral) geomagnetic latitudes than $A E$ (Thomsen, 2004; Mourenas et al., 2020).

Although ap cannot provide an accurate ranking or quantification of the maximum $\mathrm{d} H / \mathrm{d} t$ values reached during the most disturbed events due to its intrinsic saturation at $K p=9$ and its coarse 3-h time resolution, it may still provide rough estimates during less extreme events with $K p \sim 3-7$ (Kappenman, 2005). Therefore, it is worth examining whether some timeintegrated measure of ap could still be used to simply categorize disturbed/quiet days of expected stronger occurrence/absence of GIC effects at middle latitudes, during a large series of medium (most frequent) to strong (more rare) time-integrated ap events spread over 6-10 years.

Accordingly, we shall consider in Section 4.3 a third parameter of geomagnetic activity, IntAp, corresponding to the daily maximum level of the integral of 3-hourly ap values over a continuously active period of $a p \geq 15 \mathrm{nT}$ (Mourenas et al., 2019, 2020). This should allow to categorize disturbed days that include contributions to GICs from both (storm-time) ring current variations and strong substorms, usefully complementing the $\operatorname{Int}(\mathrm{d}(\mathrm{SYM}-\mathrm{H}) / \mathrm{d} t)$ and IntDst parameters. Indeed, IntAp provides a rough estimate of the effects at middle latitudes of significant time-integrated $\mathrm{d} H / \mathrm{d} t$ disturbances due to substorms, which often do not reach the low latitudes where SYM-H and Dst are measured.

In addition, we shall consider a fourth parameter, called Int $A E$, which is based on the high-latitude $A E$ auroral electrojet index Mayaud (1980). IntAE is the daily maximum level of the integral of $A E$ calculated over the same period of continuously high $a p \geq 15 \mathrm{nT}$ as IntAp (generally corresponding to $A E>200 \mathrm{nT}$ ), to ensure that the corresponding substorm-related magnetic disturbances effectively reach middle latitudes (Mourenas et al., 2019, 2020). IntAE provides a measure of 


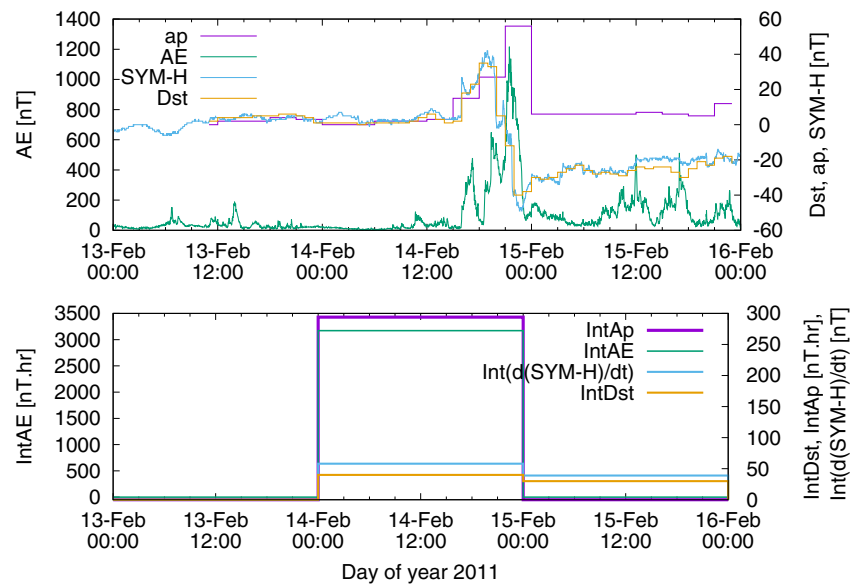

Fig. 1. Upper panel: SYM-H, Dst, $A p$, and $A E$ indices during the 13-15 February 2011 geomagnetic event. Bottom panel: corresponding $\operatorname{Int}(\mathrm{d}(\mathrm{SYM}-\mathrm{H}) / \mathrm{d} t)$ (in nT), and IntDst, IntAp, and IntAE (in nT.hr) cumulative parameters, calculated using thresholds SYM-H $\leq$ $-30 \mathrm{nT}$, Dst $\leq-30 \mathrm{nT}$, or $a p \geq 15 \mathrm{nT}$.

cumulative substorm-related disturbances, corresponding to continuous periods of auroral current variations roughly similar to High-Intensity Long-Duration Continuous $A E$ Activity (HILDCAA) events (Tsurutani et al., 2006).

These four cumulative metrics of disturbed days are displayed in Figure 1 together with 1-min SYM-H and $A E$, hourly Dst, and 3-hourly ap, during a moderate geomagnetic storm on 14-15 February 2011 that reached a minimum SYM-H = $-49 \mathrm{nT}$ and a minimum $D s t=-40 \mathrm{nT}$ on 14 February, with strong substorms (identified by peaks in $A E$ and $a p$ ) during storm sudden commencement and main phase, and with a very weak secondary minimum of $D s t$ reaching $-30 \mathrm{nT}$ on 15 February at 17 UT during a burst of $A E$ activity.

\section{Methods}

In the present follow-up study to the work by Výbošt'oková \& Švanda (2019), we search for a tighter relationship between power grid anomalies and geomagnetic storms, based on the same datasets of anomalies in the Czech power grid. We also address the important and as yet unanswered question of the time delay between geomagnetic events and the occurrences of anomalies.

Our working hypothesis is that disturbed days of high geomagnetic activity should cause an increase in daily rates of anomalies in the power distribution network as compared with quiet days. Accordingly, the daily anomaly rates should sharply peak within a few days (with some delay) after such disturbed days, and then decrease back to normal levels. This corresponds to a rapid response to GICs induced by substorms and storms, as observed for a few selected events - e.g., see Gil et al. (2019); Wang et al. (2015).

Unfortunately, in a mid-latitude country such as the Czech Republic, the effects of geomagnetic activity are expected to be weak. Consequently, an investigation of individual, moderate geomagnetic events is not expected to reveal a significant increase of anomalies, because such anomalies induced by geomagnetic activity (via GICs) will generally remain hidden among many other anomalies caused by various other effects. It is therefore imperative in our statistical analysis to find a way to reduce the importance of anomalies caused by other effects. Note that our data series cover 6-10 years, each subset providing records of anomaly rates occurring during many separated disturbed days of high geomagnetic activity. Therefore, a feasible approach is to average over all these different events. The corresponding methodology is the Superposed Epoch Analysis, widely used in astrophysics.

A Superposed Epoch Analysis (SEA; Chree, 1913) is a statistical technique used to reveal either periodicities within a time sequence, or to find a correlation between two time series. In the later case, the method proceeds in several steps.

1. In the reference time series, occurrences of the repeated events are defined as key times (or epochs).

2. Subsets are extracted from the second time series within some range around each key time.

3. Subsets from each time series are superposed, synchronized at the same key time (Day 0), and averaged, allowing inter-comparisons.

This methodology is known to efficiently enhance the "signal" (related variations in both series) with respect to "noise" (unrelated variations in both series), because the noise adds up incoherently, whereas the signal is reinforced by the superposition.

Thus, we performed the SEA of geomagnetic activity defined by $\operatorname{Int}(\mathrm{d}(\mathrm{SYM}-\mathrm{H}) / \mathrm{d} t)$ or IntDst parameters. A range of event thresholds SYM-H (or Dst) $<-25 \mathrm{nT}$ to $-50 \mathrm{nT}$ was considered, to keep only periods corresponding to weak to large geomagnetic storms (Gonzalez et al., 1994) and to allow for the determination of the best thresholds on event strength. Other days were assigned a zero level of $\operatorname{Int}(\mathrm{d}(\mathrm{SYM}-\mathrm{H}) / \mathrm{d} t)$ or IntDst. An important further requirement was that the 5-day period immediately preceding the start of a geomagnetic storm (Day 0 in the SEA) contained a zero level of the considered geomagnetic activity parameter (that is, all such quiet days must have IntDst $=0$ or $\operatorname{Int}(\mathrm{d}(\mathrm{SYM}-\mathrm{H}) / \mathrm{d} t)=0)$. This rather strict constraint should allow to better quantify the effect of geomagnetic storms on the power grid during disturbed days as compared with quiet days, at the expense of a slight reduction of the number of considered events. In a second step, we analyzed in more details these SEAs to determine as accurately as possible the time delay (after the start of a storm) that corresponds to the statistically most significant increase of anomalies, for each type of power grid equipment.

\section{Results of superposed epoch analysis}

A Superposed Epoch Analysis was performed based on IntDst and $\operatorname{Int}(\mathrm{d}(\mathrm{SYM}-\mathrm{H}) / \mathrm{d} t)$ parameters, considering successively thresholds $D s t<-25 \mathrm{nT},-30 \mathrm{nT},-40 \mathrm{nT}$, and $-50 \mathrm{nT}$, or SYM-H $<-25 \mathrm{nT},-30 \mathrm{nT},-40 \mathrm{nT}$, and $-50 \mathrm{nT}$, to explore the dependence of power grid anomalies on the minimum strength of geomagnetic storms. The number of epochs considered in the SEAs of each reference series are given in Table 2. 
Table 2. The number of epochs considered in SEAs for various reference series.

\begin{tabular}{lcc}
\hline Reference series & Threshold & \# of epochs \\
\hline IntDst & $-50 \mathrm{nT}$ & 138 \\
IntDst & $-40 \mathrm{nT}$ & 172 \\
IntDst & $-30 \mathrm{nT}$ & 221 \\
IntDst & $-25 \mathrm{nT}$ & 222 \\
Int $(\mathrm{d}(\mathrm{SYM}-\mathrm{H}) / \mathrm{d} t)$ & $-50 \mathrm{nT}$ & 154 \\
Int $(\mathrm{d}(\mathrm{SYM}-\mathrm{H}) / \mathrm{d} t)$ & $-40 \mathrm{nT}$ & 191 \\
Int $(\mathrm{d}(\mathrm{SYM}-\mathrm{H}) / \mathrm{d} t)$ & $-30 \mathrm{nT}$ & 218 \\
Int $(\mathrm{d}(\mathrm{SYM}-\mathrm{H}) / \mathrm{d} t)$ & $-25 \mathrm{nT}$ & 231 \\
\hline
\end{tabular}

The SEAs obtained for IntDst and Int (d(SYM-H)/dt) both show a clear peak of geomagnetic activity at Day 0 and a sharp decrease on Day 1 for IntDst or on Day 2 for $\operatorname{Int}(\mathrm{d}(\mathrm{SYM}-\mathrm{H}) / \mathrm{d} t)$. The later decrease for $\operatorname{Int}(\mathrm{d}(\mathrm{SYM}-\mathrm{H}) / \mathrm{d} t)$ is due to the presence of significant $\mathrm{d}(\mathrm{SYM}-\mathrm{H}) / \mathrm{d} t$ variations during the recovery phase of many storms stretching over at least two consecutive days, whereas IntDst is generally calculated only during storm main phase. Figure 2 shows the SEAs obtained for the D12 series (power lines). Similar trends are found for other datasets concerning power lines. All the figures corresponding to the different series D1-D12 are available in the Appendix as Figures A.1-A.12.

\subsection{Storm effects: 5-day periods after/before day 0}

Next, we compared the period of 5 disturbed days immediately following Day 0 (the day of peak storm activity) with the 5 -day period immediately preceding Day $0-$ a preceding period of quiet days especially selected to have zero IntDst or $\operatorname{Int}(\mathrm{d}(\mathrm{SYM}-\mathrm{H}) / \mathrm{d} t)$ levels. This allows to directly check the impact of disturbed days of geomagnetic storms on power grid anomalies, as compared with quiet days. For the two time intervals, we summed the total number of registered anomalies in the superposed series for each data subset and computed the statistical significance of the differences using the standard binomial statistical test. We tested the null hypothesis that the number of anomalies recorded over quiet days is not different from the number of anomalies recorded over disturbed days, that is, the null hypothesis that the probability of recording anomalies is the same during quiet and disturbed days. Should the resulting $p$-value be smaller than the selected statistical threshold (usually 0.05 for single-bin tests), we reject the null hypothesis, thereby saying that the recorded differences are indeed statistically significant.

The results, summarized in Table 3 , reveal a clear increase of anomalies during the period of 5 disturbed days following Day 0 as compared with the period of 5 quiet days preceding Day 0, for the two series D11 and D12 corresponding to power lines. The number of anomalies increases by $5 \%$ for D12 and by $30 \%$ for D11, with corresponding $p$-values always statistically significant $(<0.05)$, for thresholds $<-30 \mathrm{nT}$ or $<-40 \mathrm{nT}-$ except for $\operatorname{Int}(\mathrm{d}(\mathrm{SYM}-\mathrm{H}) / \mathrm{d} t)$ and D11 for a threshold $<-30 \mathrm{nT}$. Lower or higher thresholds usually lead to less statistically significant increases of anomalies, although not always - e.g. for D11 and IntDst, the $<-25 \mathrm{nT}$ threshold gives a higher statistical significance. This means that moderate events with minimum Dst or SYM-H near $-40 \mathrm{nT}$ have often a statistically detectable impact on anomaly rates, whereas weaker events do not. The same thresholds also lead to the highest peaks of anomalies after Day 0 in many other series. Finally, for D11 and D12, the $<-40 \mathrm{nT}$ thresholds lead to the smallest $p$-values $(<0.01)$ for both IntDst and $\operatorname{Int}(\mathrm{d}(\mathrm{SYM}-\mathrm{H}) / \mathrm{d} t)$, as well as to the smallest $p$-values $<0.1-0.2$ for D8 and D10 when considering IntDst, and to the smallest or second smallest $p$-values $<0.2-0.36$ for D2 and D9 when considering Int $(\mathrm{d}(\mathrm{SYM}-\mathrm{H}) / \mathrm{d} t)$. Therefore, the thresholds SYM-H $<-40 \mathrm{nT}$ and $D s t<-40 \mathrm{nT}$ are probably the most appropriate to detect statistically significant increases of anomalies related to geomagnetic storms.

The weaker significance of results for higher thresholds $<-25 \mathrm{nT}$ agrees with previous observations from Lotz \& Danskin (2017) that weaker events have little effects on induced electric fields. However, moderate Dst or SYM-H geomagnetic disturbances in the range $-40 \mathrm{nT}$ to $-50 \mathrm{nT}$ are found to still have some impact on power lines. The weaker significance of results for lower thresholds $<-50 \mathrm{nT}$ is likely due to a combination of two different effects: (i) storms start slightly later when using a threshold $<-50 \mathrm{nT}$ than for higher thresholds $<-40 \mathrm{nT}$ or $<-30 \mathrm{nT}$, meaning that the 5-day period preceding Day 0 can actually contain significant $\mathrm{d} H / \mathrm{d} t$ geomagnetic activity leading to some anomalies, and (ii) the $<-50 \mathrm{nT}$ threshold corresponds to a $30 \%-40 \%$ smaller number of events than the $<-30 \mathrm{nT}$ threshold, decreasing the sensibility of the SEA to a potential slight increase of anomalies due to storms.

A detailed inspection of the SEAs of D12 lends further credence to the impact of geomagnetic storms on power lines. Indeed, for both IntDst and $\operatorname{Int}(\mathrm{d}(\mathrm{SYM}-\mathrm{H}) / \mathrm{d} t)$, the peaks of anomalies in the few days following Day 0 reach the highest daily levels of anomalies of the whole 21-day SEAs for $<-30 \mathrm{nT}$ to $<-50 \mathrm{nT}$ thresholds, the main increases of anomalies occurring from Day +0 to Day +3 . The 4 -day period preceding Day 0 also contains less anomalies than other periods. For D1 1 and thresholds $<-30 \mathrm{nT}$ to $<-40 \mathrm{nT}$, the 4-day period following Day 0 has also the highest number of anomalies of the whole 21-day SEA, while the 5-day interval preceding Day 0 has the lowest average number of anomalies of the whole SEA.

\subsection{Storm effects: 3-day periods before/after day 0 with time lags}

Next, we examined in more details the SEAs performed based on IntDst and Int $(\mathrm{d}(\mathrm{SYM}-\mathrm{H}) / \mathrm{d} t)$ parameters for thresholds Dst $<-40 \mathrm{nT}$ and SYM-H $<-40 \mathrm{nT}$. We considered two shorter 3-day periods, located before and after Day 0 . We varied the time lag between them and calculated (as before for 5-day periods) the statistical significance of the difference in anomaly rates between these two periods. Considering shorter 3-day periods should help to determine more precisely the (statistically most significant) time delay between the start of a geomagnetic storm and the related increase of the number of anomalies.

Figure 3 for D12, Figure 4 for D8, and Figures A.13-A.24 in the Appendix for all other datasets, show two-dimensional maps of the increases (or decreases) of the number of anomalies as a function of the middle day of the first and second 3-day periods, together with maps of the corresponding $p$-values computed only for increases.

Let us examine these maps of $p$-values. For consistency with the procedure of estimation of the statistical significance 

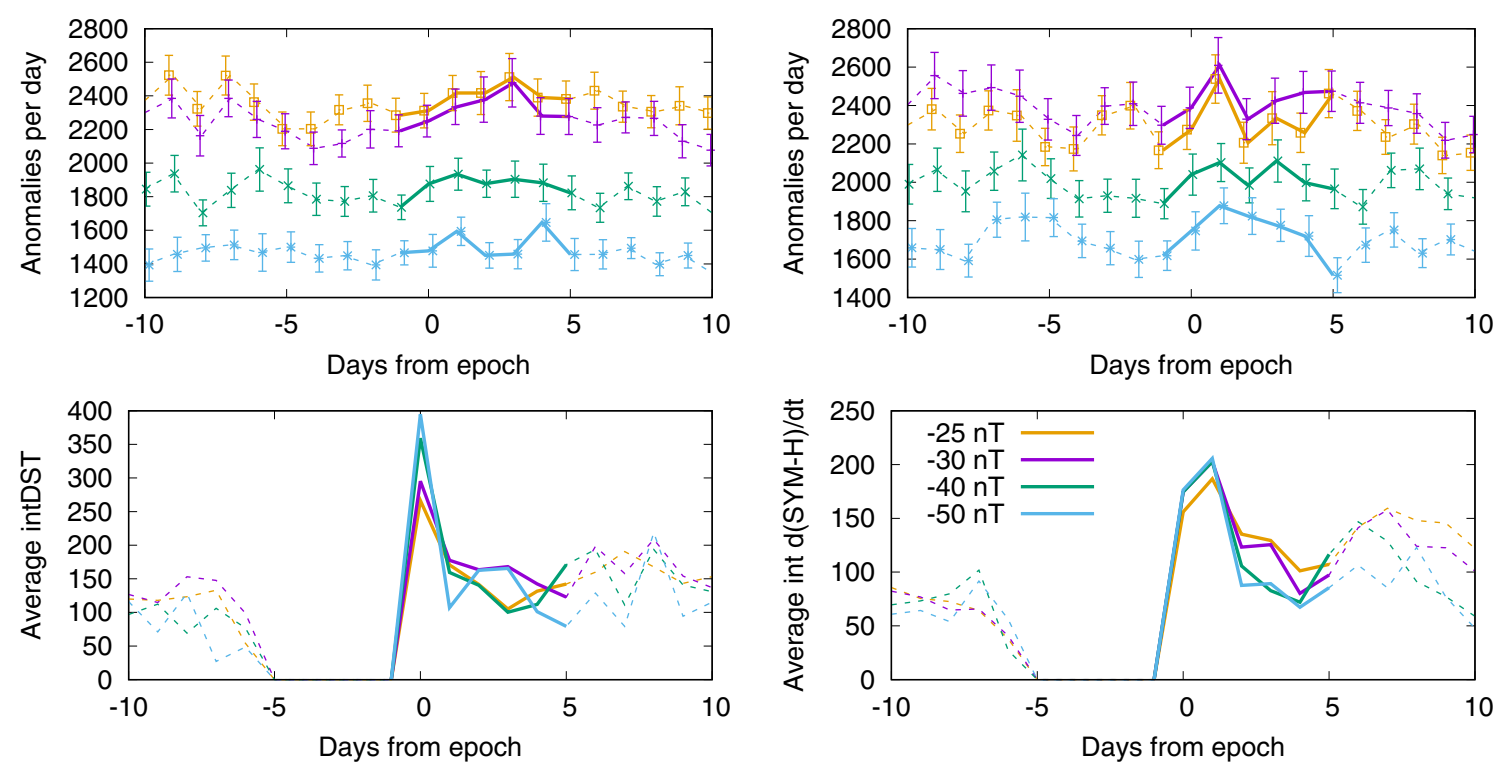

Fig. 2. Plots of epoch-superposed daily numbers of anomalies in the D12 series, considering IntDST (in nT·hr, left) and Int(d(SYM-H)/dt) (in nT, right) for different upper thresholds on Dst and SYM-H. Solid lines indicate the superposed anomaly rates (upper row) or geomagnetic activity in the reference time series (lower row) during Days -1 to +5 from the epoch (Day 0 ), whereas dashed lines show the same quantities for the remaining days. Error bars show the one-standard-deviation half-widths.

Table 3. Comparison of the number of power grid anomalies in the 5-day period prior to Day $0 \mathrm{~N}-$ and in the 5-day period after Day $0 \mathrm{~N}+$, together with $p$-values of the statistical significance of the differences. These values are given for different reference series involved in SEAs with varying thresholds.

\begin{tabular}{|c|c|c|c|c|c|c|c|c|c|c|c|c|}
\hline \multirow[t]{2}{*}{ ID } & \multicolumn{3}{|c|}{$<-25 \mathrm{nT}$} & \multicolumn{3}{|c|}{$<-30 \mathrm{nT}$} & \multicolumn{3}{|c|}{$<-40 \mathrm{nT}$} & \multicolumn{3}{|c|}{$<-50 \mathrm{nT}$} \\
\hline & $N-$ & $N+$ & $p$ & $N-$ & $N+$ & $p$ & $N-$ & $N+$ & $p$ & $N-$ & $N+$ & $p$ \\
\hline \multicolumn{13}{|c|}{ IntDst } \\
\hline D1 & 60 & 59 & 1.0 & 54 & 52 & 0.92 & 35 & 33 & 0.90 & 29.0 & 36.0 & 0.46 \\
\hline D2 & 100 & 115 & 0.34 & 109 & 137 & 0.08 & 94 & 112 & 0.24 & 82 & 94 & 0.41 \\
\hline D3 & 17 & 17 & 1.0 & 20 & 23 & 0.76 & 16 & 22 & 0.42 & 18 & 12 & 0.36 \\
\hline D4 & 58 & 38 & 0.05 & 52 & 43 & 0.41 & 45 & 46 & 1.0 & 38 & 40 & 0.91 \\
\hline D5 & 86 & 75 & 0.43 & 91 & 84 & 0.65 & 83 & 82 & 1.0 & 71 & 68 & 0.87 \\
\hline D6 & 30 & 36 & 0.54 & 40 & 39 & 1.0 & 38 & 37 & 1.0 & 34 & 31 & 0.80 \\
\hline D7 & 134 & 132 & 0.95 & 143 & 137 & 0.77 & 115 & 120 & 0.79 & 98 & 105 & 0.67 \\
\hline D8 & 968 & 955 & 0.78 & 892 & 922 & 0.50 & 710 & 760 & 0.20 & 562 & 586 & 0.50 \\
\hline D9 & 105 & 102 & 0.89 & 95 & 112 & 0.27 & 70 & 67 & 0.86 & 44 & 53 & 0.42 \\
\hline D10 & 14292 & 14338 & 0.79 & 13245 & 13477 & 0.16 & 10791 & 11047 & 0.08 & 8601 & 8764 & 0.22 \\
\hline D11 & 415 & 494 & 0.01 & 403 & 476 & 0.02 & 302 & 387 & $<0.01$ & 247 & 297 & 0.04 \\
\hline D12 & 11366 & 12118 & $<0.01$ & 10787 & 11748 & $<0.01$ & 8965 & 9421 & $<0.01$ & 7242 & 7606 & $<0.01$ \\
\hline \multicolumn{13}{|c|}{$\operatorname{Int}(\mathrm{d}(\mathrm{SYM}-\mathrm{H}) / \mathrm{d} t)$} \\
\hline D1 & 59 & 56 & 0.85 & 59 & 58 & 1.0 & 43 & 47 & 0.75 & 32 & 37 & 0.63 \\
\hline D2 & 98 & 98 & 1.0 & 104 & 110 & 0.73 & 101 & 121 & 0.20 & 93 & 107 & 0.36 \\
\hline D3 & 20 & 15 & 0.50 & 20 & 16 & 0.62 & 15 & 20 & 0.50 & 17 & 18 & 1.0 \\
\hline D4 & 53 & 36 & 0.09 & 51 & 37 & 0.17 & 43 & 45 & 0.92 & 46 & 49 & 0.84 \\
\hline D5 & 79 & 66 & 0.32 & 83 & 70 & 0.33 & 80 & 78 & 0.94 & 83 & 77 & 0.69 \\
\hline D6 & 29 & 28 & 1.0 & 35 & 31 & 0.71 & 38 & 33 & 0.64 & 38 & 29 & 0.33 \\
\hline D7 & 115 & 118 & 0.90 & 137 & 127 & 0.58 & 116 & 122 & 0.75 & 119 & 118 & 1.0 \\
\hline D8 & 964 & 936 & 0.54 & 1005 & 964 & 0.37 & 784 & 790 & 0.90 & 635 & 667 & 0.39 \\
\hline D9 & 98 & 101 & 0.89 & 107 & 102 & 0.78 & 80 & 93 & 0.36 & 58 & 74 & 0.19 \\
\hline D10 & 14220 & 14061 & 0.35 & 14594 & 14518 & 0.66 & 11951 & 11877 & 0.64 & 9702 & 9854 & 0.28 \\
\hline D11 & 408 & 450 & 0.16 & 420 & 473 & 0.08 & 334 & 415 & $<0.01$ & 300 & 323 & 0.38 \\
\hline D12 & 11273 & 11798 & $<0.01$ & 11675 & 12305 & $<0.01$ & 9669 & 10162 & $<0.01$ & 8385 & 8714 & 0.01 \\
\hline
\end{tabular}



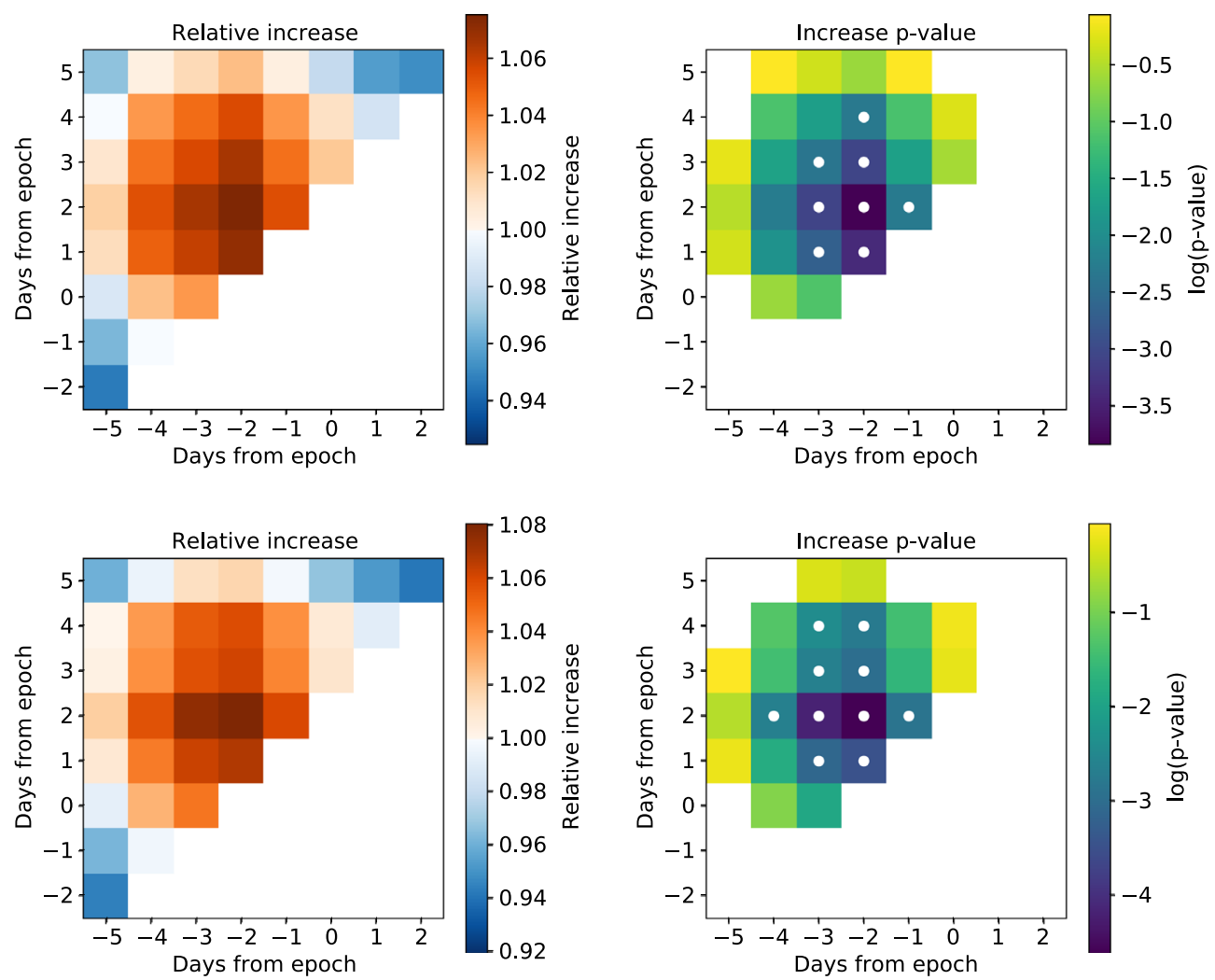

Fig. 3. (Left) Maps for D12 of increases (or decreases) of the number of anomalies as a function of the middle day of the first (abscissa) and second (ordinate) considered 3-day periods. (Right) Maps of the corresponding $p$-values. The upper row is computed for the IntDst reference series, whereas the lower row corresponds to the $\operatorname{Int}(\mathrm{d}(\mathrm{SYM}-\mathrm{H}) / \mathrm{d} t)$ reference series. The $p$-values are evaluated only if there is an increase of anomaly rates in the second 3-day period as compared to the first 3-day period. Note the logarithmic scale of the plotted $p$-values: $p=0.0055$ (the adopted level of statistical significance for individual bins) corresponds to $\log p=-2.26$. Statistically significant bins are indicated by white dots. Blank bins are indicated by the white colour.

adopted in Section 4.1, we need to compare the number of anomalies over the same 5-day periods after and before Day 0. Accordingly, we must only consider the bins (representing 3-day periods) comprised between Days -4 and - 2 (actually covering Days -5 to -1 ) for the period before Day 0 , and the bins comprised between Days +2 and +4 (actually covering Days +1 to +5 ) for the period following Day 0 . There are $3 \times 3=9$ such bins. Finding only one bin with a $p$-value $\sim 0.05$ (corresponding to a $5 \%$ probability to obtain an increase of anomalies by chance) among 9 bins is not anymore as statistically significant as before. Therefore, an individual bin (representing 3-day periods) is hereafter required to have a smaller $p$-value $\leq 0.05 / 9=0.0055$ to be considered statistically significant.

In the case of the D12 dataset (power lines), there are six bins with $p$-values $<0.0055$ for both IntDst and $\operatorname{Int}(\mathrm{d}(\mathrm{SYM}-\mathrm{H}) / \mathrm{d} t)$ in the considered square of $3 \times 3$ bins centered on $(-3,+3)$ in Figure 3 , corresponding to a statistically significant increase of anomalies. A significant increase of anomalies is already observed over final 3-day periods centered on Day +1 , as compared with initial 3-day periods centered on Days -3 and -2 , indicating an immediate effect of geomagnetic storms on power lines.

In the case of D8 (transformers), however, the three bins corresponding to increases of anomalies with the smallest $p$-values are found in Figure 4 for final 3-day periods centered on Days +3 to +4 , as compared with initial 3-day periods centered on Days -1 to 0 . Therefore, there is a clear time delay of $\sim 2-3$ days between a variation of IntDst or $\operatorname{Int}(\mathrm{d}(\mathrm{SYM}-\mathrm{H}) / \mathrm{d} t)$ and the corresponding variation of the number of anomalies in the D8 dataset. In such a situation, it is more appropriate to consider for D8 the square of $3 \times 3=9$ bins centered on $(-1,+3)$ in Figure 3 . Inside this domain, one bin has a $p$-value $=0.0045<0.0055$ for IntDst in Figure 4 , indicating a statistically significant delayed increase of anomalies for D8.

Overall, the results displayed in Figures 3-4 and in Figures A.13-A.24 therefore confirm the preceding results obtained for 5-day periods, but they further allow to determine the optimal time delays before a statistically significant increase of anomalies in different power grid equipment.

Most strikingly, a statistically highly significant increase of anomalies is found for D11-D12 (power lines) for both IntDst and $\operatorname{Int}(\mathrm{d}(\mathrm{SYM}-\mathrm{H}) / \mathrm{d} t)$ only $\sim 0-1$ day after Day 0 , and as compared with all the preceding 3-day periods without storm activity (i.e., with $\operatorname{IntDst}=0$ or $\operatorname{Int}(\mathrm{d}(\mathrm{SYM}-\mathrm{H}) / \mathrm{d} t)=0)$. Some less significant increases are also found for D4 (power lines as D11-D12) for IntDst. Such results imply an immediate effect of geomagnetic storms on power lines, already on Days 0 to +1 . This looks quite realistic, because any effect of GICs on power lines (due to harmonics-related current waveform distortion 

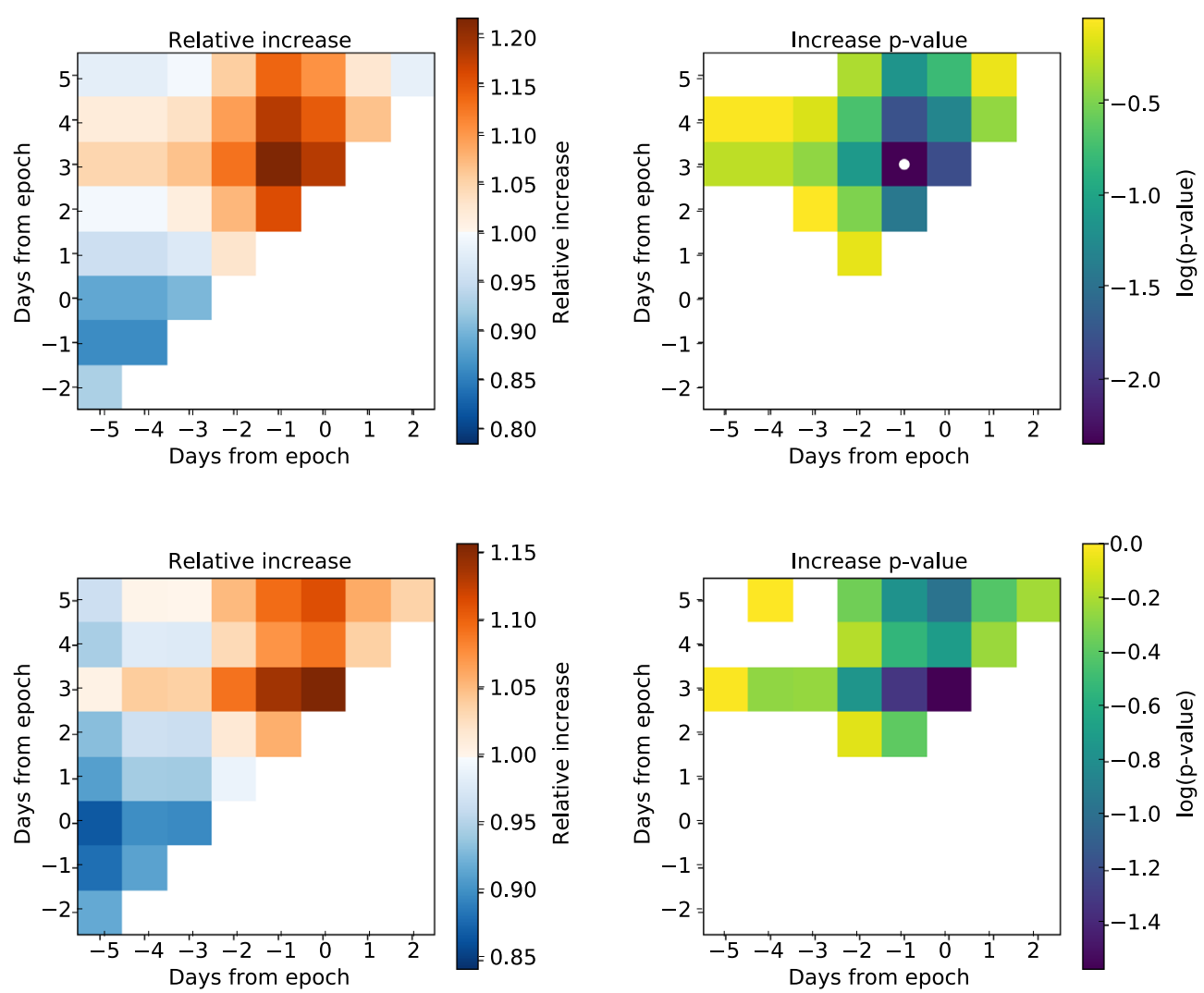

Fig. 4. (Left) Maps for D8 of increases (or decreases) of the number of anomalies as a function of the middle day of the first (abscissa) and second (ordinate) considered 3-day periods. (Right) Maps of the corresponding $p$-values. The upper row is computed for the IntDST reference series, whereas the lower row corresponds to the $\operatorname{Int}(\mathrm{d}(\mathrm{SYM}-\mathrm{H}) / \mathrm{d} t)$ reference series. The $p$-values are evaluated only if there is an increase of anomaly rates in the second 3-day period as compared to the first 3-day period. Note the logarithmic scale of the plotted $p$-values: $p=0.0055$ (the adopted level of statistical significance for individual bins) corresponds to $\log p=-2.26$. Statistically significant bins are highlighted by white dots. Blank bins are indicated by the white colour.

leading to a detrimental reaction of protective relays or other devices connected to these lines) is likely to occur almost immediately.

Furthermore, Figure 4 reveals the presence of a statistically significant delayed increase of anomalies for D8 (high voltage transformers) following geomagnetic storms when considering IntDst (an increase is also present for $\operatorname{Int}(\mathrm{d}(\mathrm{SYM}-\mathrm{H}) / \mathrm{d} t)$ but somewhat less significant), with a delay of $\sim 3$ days after Day 0 . This strongly suggests the presence of some delayed effects of storm-time geomagnetic activity on transformers (note also that the lowest rates of anomalies are observed here on Days -2 to 0 , similarly corresponding to a delayed effect of the previous days of zero storm activity). Transformers may indeed be affected by GICs but still continue to operate for a while typically for a few days - before actual problems ultimately show up and are registered in logs (e.g., Wang et al., 2015).

\subsection{Ring and auroral currents effects: IntAp parameter}

Since both ring current variations during storms and other (mainly auroral) current variations during strong substorms may produce significant GICs, we further performed similar SEAs for the IntAp parameter, which (despite its own limitations, see Sect. 2.2 and Kappenman, 2005) is expected to roughly take into account the effects of both kinds of disturbances - whereas
IntDst and $\operatorname{Int}(\mathrm{d}(\mathrm{SYM}-\mathrm{H}) / \mathrm{d} t)$ only correspond to storm periods. However, due to the relatively low threshold $a p \geq 15$ (equivalent to $K p \geq 3$ ) of integration used to calculate daily IntAp levels, this new data series contained many more events (notably, many isolated substorms, sometimes outside of storms) than the previous IntDst (storm) data set. As a result, requiring as before a 5-day period prior to events with IntAp $=0$ led to only a weak IntAp maximum on Day 0, with a preceding IntAp peak on Days -10 to -5 of comparable magnitude. Therefore, we changed our selection procedure, to consider only events with a peak IntAp $>1000 \mathrm{nT}$ hr and such that no similar event was present in the preceding 5 days.

The resulting SEAs displayed in Figure 5 show that this new selection procedure produces a large peak IntAp $\sim 1400 \mathrm{nT} \mathrm{hr}$ on Day 0 in the SEAs, with much lower levels on all 10 previous days, especially between Days -6 and -2 . The daily number of anomalies is found to increase by a statistically very significant amount during the 5-day period following Day 0 as compared to the 5-day period preceding Day 0, for series D11 and D12 in Figure 5, with corresponding $p$-values 0.03 and 0.007 , respectively. There is a remarkable simultaneity between the peak of IntAp and the peak of anomalies in the two SEAs with at most one day of delay. Moreover, such peaks of daily anomalies on Days 0 or +1 are consistently larger than all other daily values in the full 21-days SEAs. Such results therefore demonstrate 

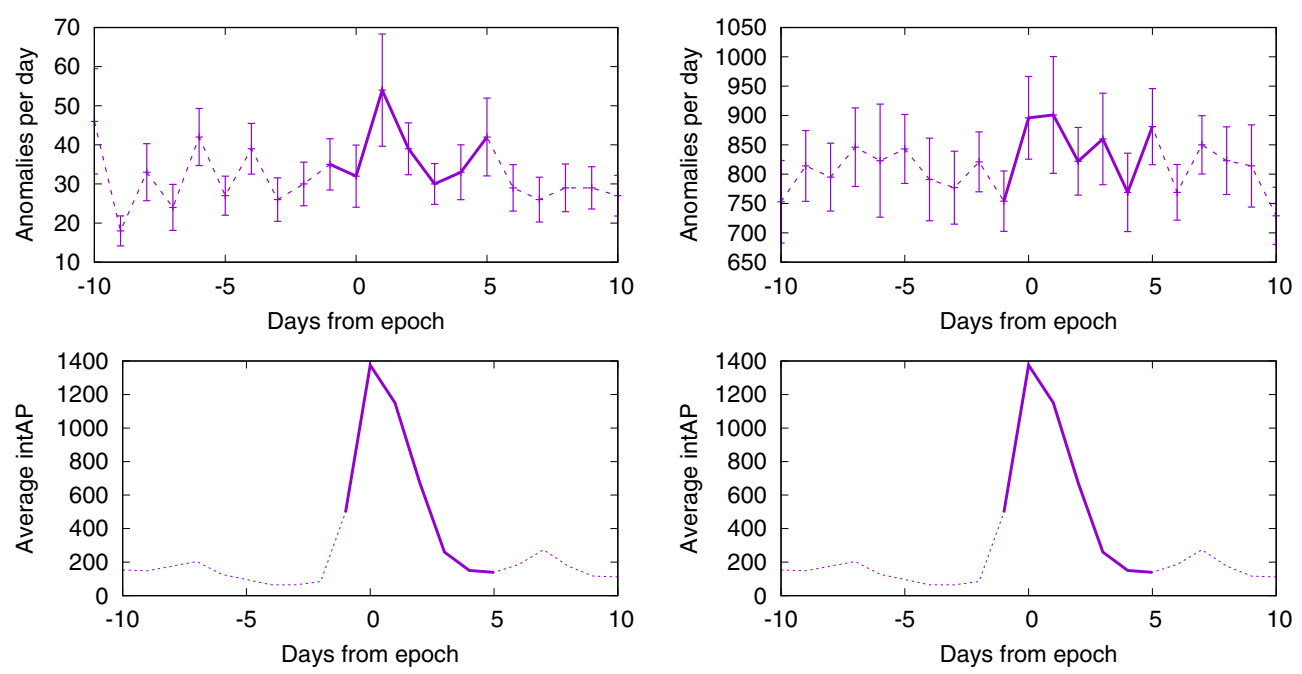

Fig. 5. Plots of epoch-superposed subsets D11 (left) and D12 (right) of variations of the daily number of anomalies as a function of time, considering the IntAp parameter. Solid lines indicate mean superposed daily rates of anomalies (upper row) or geomagnetic activity IntAp (in $\mathrm{nT}$ h) in the reference time series (bottom row) during Days -1 to +5 from the epoch (Day 0), whereas dashed lines show the same quantities for the remaining days. Error bars show the one-standard-deviation half-widths.

the likely presence of nearly immediate effects of both stormrelated and substorm-related geomagnetic disturbances on GICs and power lines (D11-D12) in the Czech power network. This is certainly due to the major impact of strong substorms on GICs, both during and outside geomagnetic storms.

There are also detectable increases of daily anomalies between 5-day periods before/after Day 0 for D8 (transformers, with a delay of $\sim 2$ days) and for D4 (power lines, immediate), but they are not statistically significant, with $p$-values $\simeq 0.25$ (see SEAs for all D1-D12 series provided in Figs. A.25-A.36 in the Appendix).

Besides, there is a statistically significant increase of anomalies for D10 (high and very high voltage electrical substations) with a $p$-value of 0.006 , with a first peak of anomalies at Day +1 but a much delayed higher peak on Days +4 and +5 . While power lines react immediately to GICs, high and very high voltage electrical substations, which comprise busbars, capacitors, or transformers, may indeed be affected but still continue to operate without registered problems until the cumulative damage reaches a sufficient level. A time lag of 3-5 days does not seem wholly unrealistic in this respect (Kappenman, 2007; Wang et al., 2015).

It is worth noting that our previous analysis based on IntDst did not show a statistically significant impact of storms for D10 (although the smallest $p$-value reached 0.08 in Tab. 3), contrary to the present analysis based on IntAp. This suggests that prolonged 2-3 day periods of repeated non-storm-time substorms or solar wind sudden impulses (SIs), taken into account by IntAp but not by IntDst, could have a noticeable effect on some electrical substations.

\subsection{Auroral current effects: IntAE parameter}

Next, we performed similar SEAs for the IntAE parameter that provides a measure of cumulated high-latitude auroral current variations. An increased hourly auroral electrojet index $A E>150-250 \mathrm{nT}$ is one of the dominant manifestations of substorms, and many substorm studies rely on $A E$ to estimate the intensity of substorms, although $A E$ is not a specific measure of substorms (Kamide \& Kokobun, 1996; Tsurutani et al., 2004). We compared the period of 5 disturbed days (with daily IntAE $>150 \mathrm{nT} \mathrm{hr}$ ) immediately following Day 0 (the day of peak IntAE) with the 5-day period immediately preceding Day $0-$ a preceding period of nearly quiet days (with daily IntAE $<30 \mathrm{nT} \mathrm{hr}$ ) especially selected to have such nearly zero IntAE levels. This way, we can check the impact of disturbed days of strong $A E$ activity (often corresponding to substorms, occurring both during and outside storms) on power grid anomalies, as compared with quiet days. We also tried as before to consider shorter 3-day periods to help determine the best time lags between increases of anomalies and Day 0 .

All the corresponding plots are given in Figures A.37-A.48 in the Appendix. In general, these results mostly agree with the IntAp results. However, they are somewhat less statistically significant than the results obtained with all the preceding metrics, except for the D11-D12 (power lines) series. For D11, we find a statistically significant $15 \%$ increase in the total number of anomalies after/before Day 0, with a $p$-value of 0.034 (see Fig. 6), while for D12 (power lines) the increase of anomalies is only $2.6 \%$, with a barely significant $p=0.055$.

An important point is that these results based on IntAE confirm the impact on power lines of auroral electrojet disturbances, often related to substorms. Nevertheless, these results also suggest that the IntDst, IntAp, or Int (d(SYM-H)/dt) metrics may be slightly more appropriate than IntAE for categorizing disturbed days leading to GIC effects at middle latitudes in the Czech power grid. This could stem from the higher latitudes of stations measuring $A E$ than for the mid-latitude $a p$ index: IntAE may either take into account weak substorms that actually do not strongly affect middle latitudes, or it may under-estimate mid-latitude disturbances produced by large substorms (Thomsen, 2004; Lockwood et al., 2019). Alternatively, there could be some significant impacts of ring current variations on GICs at mid-latitudes, not taken into account in IntAE. 

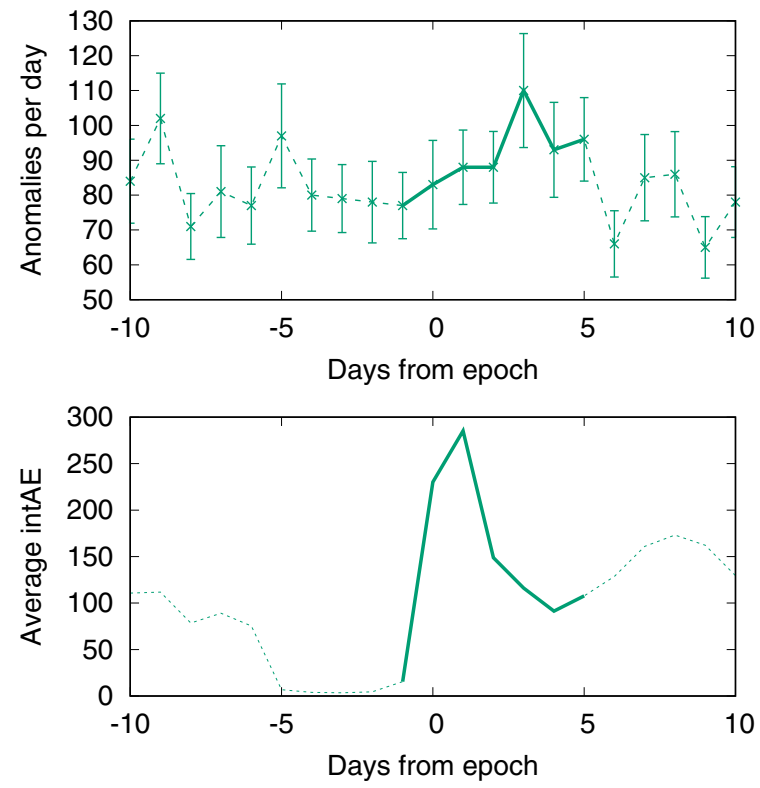

Fig. 6. Epoch-superposed daily numbers of anomalies in the D11 series, considering the IntAE parameter. Solid lines indicate superposed anomaly rates (upper panel) or IntAE (in $\mathrm{nT} \mathrm{h}$ ) in the reference time series (lower panel) during Days -1 to +5 from the epoch (Day 0 ), whereas dashed lines show the same quantities for the remaining days. Error bars show one-standard-deviation half-widths.

\section{Discussion}

In the SEAs, roughly $\approx 5-10 \%$ increases of the number of anomalies were often observed during the five most disturbed days as compared with the preceding five consecutive quiet days. However, it is important to note that such increases of anomalies were present during only the five most disturbed days among the 21-day total duration of each SEA. It is also unclear if there was any statistically significant increase of anomalies caused by the much weaker geomagnetic activity present during other days that did not fulfill the criteria for our SEA analysis. It is thus difficult to obtain a credible estimate of the total fraction of anomalies that could be directly related to geomagnetic effects. In our previous study (Výbošt'oková \& Švanda, 2019), the corresponding total number of anomalies attributable to variations of geomagnetic activity was estimated as $1-4 \%$. Such values are consistent with results from a previous study of the impact of solar activity on the US electric power transmission network in 1992-2010, which showed that $\sim 4 \%$ of the corresponding anomalies were likely attributable to strong geomagnetic activity and GICs (Schrijver \& Mitchell, 2013).

We also considered different parameter series, namely cumulative IntDst, IntAp, and IntAE parameters integrated over the preceding 5 or 10 days, to evaluate the effects of a longer exposure to GICs on power-grid devices. The corresponding superposed epoch analysis did not yield statistically significant results. Without a proper event selection procedure and no integration limit, the SEAs were dominated by weak events, during which the effects were probably weak and did not emerge from the average rates of anomalies due to causes other than geomagnetic activity. SEAs were further performed separately for weak, moderate, and strong events, but this did not significantly improve the results. The most promising results in terms of magnitude of increase of anomalies during stronger activity were for D8, D10, and D12 for IntDst (with lags of 1-3 days), and D8 and D11 for IntAE.

Based on our analysis, it turns out that geomagnetic disturbances affected mostly the datasets registering anomalies on power lines. It is interesting to note that most of the power lines in D7, D11, and D12 are the power lines with distances between grounding points of the order of tens of kilometers. We also found significant delayed effects in the D8 dataset of highvoltage transformers. Although significant effects were observed in D4 during strong storms (see Fig. A.40), the distances between grounding points are of the order of hundreds of meters in this case, that is, much shorter than for the other power-line datasets. The topology of the network in D4 is also far more complex than in the other power-line datasets. It is unlikely that GICs induced in the D4 network could be responsible for the observed increase of anomaly rate after Day 0 in the corresponding SEA. Nevertheless, some detrimental currents could have entered the D4 network from nearby connected networks of other power companies and caused operational anomalies during strong events.

\section{Conclusions}

As noted by Schrijver \& Mitchell (2013), the selection of an appropriate geomagnetic parameter is very important when searching for correlations between anomalies recorded in human infrastructures and variations of geomagnetic activity. Here, we have presented results obtained by considering four different and complementary parameters of cumulative geomagnetic activity, namely the different storm-time $\operatorname{Int}(\mathrm{d}(\mathrm{SYM}-\mathrm{H}) / \mathrm{d} t)$ and IntDst low-latitude metrics tracking mainly ring current variations, the high-latitude Int $A E$ metric mainly tracking auroral current variations, and the mid-latitude IntAp metric tracking both ring and auroral current variations - all of which were integrated over geomagnetically disturbed periods. This allowed us to compare the cumulated number of anomalies observed in the Czech power grid during the corresponding disturbed days of high geomagnetic activity with the number of anomalies recorded during quiet days.

At the considered middle geomagnetic latitudes, our statistical analysis of $\sim 10$ years of data has shown that space weatherrelated events affected mostly long power lines (D11, D12), probably due to a distortion of the electrical current waveform that eventually triggered a detrimental reaction of protective relays or disrupted other connected devices. However, significant and slightly more delayed (by $\sim 1-2$ days) effects were also observed in high-voltage transformers.

Both substorm-related disturbances and magnetic storms were found to have statistically significant impacts on the power grid network, since the four considered measures of disturbed days $(\operatorname{Int} D s t, \operatorname{Int}(\mathrm{d}(\mathrm{SYM}-\mathrm{H}) / \mathrm{d} t), \operatorname{Int} A p$, and $\operatorname{Int} A E)$ led to more or less similar results - although IntAE was slightly less efficient. In addition, we found that considering moderate thresholds (neither too large nor too small) on time-integrated geomagnetic activity quantified by $\operatorname{Int} D s t, \operatorname{Int}(\mathrm{d}(\mathrm{SYM}-\mathrm{H}) / \mathrm{d} t)$, or IntAp, produced the most statistically significant increases 
in anomaly rates, suggesting a non-negligible impact of moderate disturbances. These results are therefore consistent with a major impact of substorms, either inside or outside storms, on GICs at middle latitudes, together with a possible additional impact of ring current variations during storms.

It is worth noting that our study showed that in the 5-day period following the commencement of geomagnetic activity there is an approximately $5-10 \%$ increase in the recorded power line and transformers anomalies in the Czech power grid, probably related to geomagnetic activity and GICs. Such values are consistent with previous results concerning the US power grid (Schrijver \& Mitchell, 2013). Schrijver et al. (2014) further found that for the US network, the 5\% stormiest days were apparently the most dangerous, with a $20 \%$ increase of gridrelated anomalies as compared to quiet periods. We similarly found that the days with a minimum $D s t<-50 \mathrm{nT}$ (roughly representing the $\approx 8 \%$ stormiest days, see Gonzalez et al., 1994) had probably the strongest impact in the Czech power grid, leading to immediate or slightly delayed $\sim 5-20 \%$ increases of anomalies as compared to quiet periods.

Acknowledgements. M.Š was supported by the institute research project ASU:67985815. We are grateful to power grid data providers for giving us an opportunity to exploit their logs of anomalies, namely to P. Spurný (ČEPS), J. Brož and J. Buřič (ČEZ Distribuce), R. Hanuš (PREdistribuce), and D. Mezera and R. Bílý (E.ON Distribuce). The maintenance logs are considered strictly private by the power companies and are provided under non-disclosure agreements. We gratefully acknowledge the World Data Center in Kyoto and the Space Physics Data Facility (SPDF) at NASA Goddard Space Flight Center for the OMNI data at http://omniweb.gsfc.nasa. gov of Dst and SYM-H geomagnetic indices used in this paper. Author contributions: DM designed the study and provided processed geomagnetic data, $\mathrm{KZ}$ and $\mathrm{TV}$ wrote the processing code as parts of their student projects under the supervision of MŚ. MŠ performed the analysis. DM and MS interpreted the data and wrote the manuscript. All authors contributed to the final version of paper. The editor thanks Mike Hapgood and Neil Rogers for their assistance in evaluating this paper.

\section{References}

Bailey RL, Halbedl TS, Schattauer I, Römer A, Achleitner G, Beggan CD, Wesztergom V, Egli R, Leonhardt R. 2017. Modelling geomagnetically induced currents in midlatitude Central Europe using a thin-sheet approach. Ann Geophys 35(3): 751761. https://doi.org/10.5194/angeo-35-751-2017.

Balan N, Batista IS, Tulasi Ram S, Rajesh PK. 2016. A new parameter of geomagnetic storms for the severity of space weather. Geosci Lett 3: 3. https://doi.org/10.1186/s40562-016-0036-5.

Balan N, Skoug R, Tulasi Ram S, Rajesh PK, Shiokawa K, Otsuka Y, Batista IS, Ebihara Y, Nakamura T. 2014. CME front and severe space weather. J Geophys Res Space Phys 119: 1004110058. https://doi.org/10.1002/2014JA020151.

Boteler DH. 2019. A 21st century view of the March 1989 magnetic storm. Space Weather 17: 1427-1441. https://doi.org/10.1029/ 2019SW002278.
Carter BA, Yizengaw E, Pradipta R, Halford AJ, Norman R, Zhang K. 2015. Interplanetary shocks and the resulting geomagnetically induced currents at the equator. Geophys Res Lett 42: 6554-6559. https://doi.org/10.1002/2015GL065060.

Chapman SC, Bartels J. 1940. Geomagnetism, Vol. I: Geomagnetic and Related Phenomena, Oxford University Press, London, UK.

Chree C. 1913. Some phenomena of sunspots and of terrestrial magnetism at Kew observatory. Philos Trans $R$ Soc London Ser A 212: 75-116. https://doi.org/10.1098/rsta.1913.0003.

Dungey JW. 1961. Interplanetary magnetic field and the auroral zones. Phys Rev Lett 6: 47-48. https://doi.org/10.1103/PhysRevLett.6.47.

Ganushkina NY, Jaynes A, Liemohn MW. 2017. Space weather effects produced by the ring current particles. Space Sci Rev 212: 1315-1344. https://doi.org/10.1007/s11214-017-0412-2.

Gaunt C, Coetzee G. 2007. Transformer failures in regions incorrectly considered to have low GIC risk. Proceedings from IEEE Lausanne PowerTech, pp. 807-812. https://doi.org/10.1109/ PCT.2007.4538419.

Gaunt CT. 2014. Reducing uncertainty - responses for electricity utilities to severe solar storms. J Space Weather Space Clim 4: A01. https://doi.org/10.1051/swsc/2013058.

Gil A, Modzelewska R, Moskwa S, Siluszyk A, Siluszyk M, Wawrzynczak A, Zakrzewska S. 2019. Does time series analysis confirms the relationship between space weather effects and the failures of elec- trical grids in South Poland? J Math Indus 9(1): 7. https://doi.org/10.1186/s13362-019-0064-9.

Gonzalez WD, Joselyn JA, Kamide Y, Kroehl HW, Rostoker G, Tsurutani BT, Vasyliunas VM. 1994. What is a geomagnetic storm? J. Geophys. Res. 99: 5771-5792. https://doi.org/10.1029/ 93JA02867.

Kamide Y, Kokobun S. 1996. Two-component auroral electrojet: Importance for substorm studies. Journal of Geophysical Research 101(A6): 13027-13046. https://doi.org/10.1029/96JA00142.

Kamide Y, Rostoker G. 2004. What is the physical meaning of the AE index? Eos, Trans Am Geophys Union 85(19): 188-192. https://doi.org/10.1029/2004EO190010.

Kappenman JG. 2003. Storm sudden commencement events and the associated geomagnetically induced current risks to ground-based systems at low-latitude and mid-latitude locations. Space Weather 1(3): 1016. https://doi.org/10.1029/2003SW000009.

Kappenman JG. 2005. An overview of the impulsive geomagnetic field disturbances and power grid impacts associated with the violent Sun-Earth connection events of 29-31 October 2003 and a comparative evaluation with other contemporary storms. Space Weather 3: S08C01. https://doi.org/10.1029/2004SW000128.

Kappenman JG. 2007. Geomagnetic disturbances and impacts upon power system operation, 16.1-16.22. CRC Press/IEEE Press. https://doi.org/10.1201/9781420009255.ch16.

Kataoka R, Pulkkinen A. 2008. Geomagnetically induced currents during intense storms driven by coronal mass ejections and corotating interacting regions. J Geophys Res Space Phys 113(A3): A03S12. https://doi.org/10.1029/2007JA012487.

Kepko L, McPherron RL, Amm O, Apatenkov S, Baumjohann W, Birn J, Lester M, Nakamura R, Pulkkinen TI, Sergeev V. 2014. Substorm current wedge revisited. Space Sci Rev 190: 1-46. https://doi.org/10.1007/s11214-014-0124-9.

Kikuchi T, Tsunomura S, Hashimoto S, Nozaki K. 2001. Fieldaligned current effects on midlatitude geomagnetic sudden commencements. J Geophys Res 106(A8): 15555-15566. https://doi. org/10.1029/2001JA900030.

Lakhina GS, Tsurutani BT. 2016. Geomagnetic storms: historical perspective to modern view. Geosci Lett 3(1): 5. https://doi.org/ 10.1186/s40562-016-0037-4. 
Lockwood M, Bentley SN, Owens MJ, Barnard LA, Scott CJ, Watt CE, Allanson O. 2019. The development of a space climatology: 1. Solar wind magnetosphere coupling as a function of timescale and the effect of data gaps. Space Weather 17: 133-156. https://doi. org/10.1029/2018SW001856.

Lotz SI, Danskin DW. 2017. Extreme value analysis of induced geoelectric field in South Africa. Space Weather 15: 1347-1356. https://doi.org/10.1002/2017SW001662.

Marshall RA, Dalzell M, Waters CL, Goldthorpe P, Smith EA. 2012. Geomagnetically induced currents in the New Zealand power network. Space Weather 10(S08): 003. https://doi.org/10.1029/ 2012SW000806.

Mayaud PN. 1980. Derivation, meaning, and use of geomagnetic indices. Washington DC American Geophysical Union Geophysical Monograph Series 22: 607. https://doi.org/10.1029/GM022.

McPherron RL, Chu X. 2017. The mid-latitude positive bay and the MPB index of substorm activity. Space Sci Rev 206: 91-122. https://doi.org/10.1007/s11214-016-0316-6.

Molinski TS. 2002. Why utilities respect geomagnetically induced currents. J Atmos Sol-Terr Phys 64: 1765-1778. https://doi.org/ 10.1016/S1364-6826(02)00126-8.

Mourenas D, Artemyev AV, Zhang X-J. 2018. Statistics of extreme time-integrated geomagnetic activity. Geophys Res Lett 45: 502510. https://doi.org/10.1002/2017GL076828.

Mourenas D, Artemyev AV, Zhang X-J. 2019. Impact of significant time-integrated geomagnetic activity on $2-\mathrm{MeV}$ electron flux. $J$ Geophys Res Space Phys 124: 4445-4461. https://doi.org/10.1029/ 2019JA026659.

Mourenas D, Artemyev AV, Zhang X-J. 2020. Dynamical properties of peak and time-integrated geomagnetic events inferred from sample entropy. J Geophys Res Space Phys 125(e27): 599. https://doi.org/10.1029/2019JA027599.

Pirjola R. 2000. Geomagnetically induced currents during magnetic storms. IEEE Trans Plasma Sci 28(6): 1867-1873. https://doi.org/ 10.1109/27.902215.

Pokhrel S, Nguyen B, Rodriguez M, Bernabeu E, Simpson JJ. 2018. A finite difference time domain investigation of electric field enhancements along ocean-continent boundaries during space weather events. J Geophys Res Space Phys 123(6): 5033-5046. https://doi.org/10.1029/2017JA024648.

Pulkkinen A, Bernabeu E, Thomson A, Viljanen A, Pirjola R, et al. 2017. Geomagnetically induced currents: Science, engineering, and applications readiness. Space Weather 15(7): 828-856. https://doi.org/10.1002/2016SW001501.

Schrijver CJ, Dobbins R, Murtagh W, Petrinec SM. 2014. Assessing the impact of space weather on the electric power grid based on insurance claims for industrial electrical equipment. Space Weather 12(7): 487-498. https://doi.org/10.1002/2014SW001066.

Schrijver CJ, Mitchell SD. 2013. Disturbances in the US electric grid associated with geomagnetic activity. J Space Weather Space Clim 3: A19. https://doi.org/10.1051/swsc/2013041.

Thomsen MF. 2004. Why Kp is such a good measure of magnetospheric convection. Space Weather 2(S11): 004. https://doi.org/ 10.1029/2004SW000089.
Torta JM, Serrano L, Regué JR, Sánchez AM, Roldán E. 2012. Geomagnetically induced currents in a power grid of northeastern Spain. Space Weather 10: S06002. https://doi.org/10.1029/ 2012 SW000793.

Tozzi R, De Michaelis P, Coco I, Giannattasio F. 2019. A Preliminary Risk Assessment of Geomagnetically Induced Currents over the Italian Territory. Space Weather 17: 46-58. https://doi.org/10.1029/2018SW002065.

Tsurutani BT, Gonzalez WD, Gonzalez ALC, Guarnieri FL, Gopalswamy N, et al. 2006. Corotating solar wind streams and recurrent geomagnetic activity: A review. J Geophys Res 111: A07S01. https://doi.org/10.1029/2005JA011273.

Tsurutani BT, Gonzalez WD, Guarnieri F, Kamide Y, Zhou X, Arballo JK. 2004. Are high- intensity long-duration continuous AE activity (HILDCAA) events substorm expansion events? J Atmos Sol-Terr Phys 66(2): 167-176. https://doi.org/10.1016/j.jastp.2003.08.015.

Tsurutani BT, Verkhoglyadova OP, Mannucci AJ, Lakhina GS, Li G, Zank GP. 2009. A brief review of "solar flare effects" on the ionosphere. Radio Sci 44(A5): RS0A17. https://doi.org/10.1029/ 2008RS004029.

Turnbull KL, Wild JA, Honary F, Thomson AWP, McKay AJ. 2009. Characteristics of variations in the ground magnetic field during substorms at mid latitudes. Ann Geophys 27: 3421-3428. https://doi.org/10.5194/angeo-27-3421-2009.

Verscharen D, Klein KG, Maruca BA. 2019. The multi-scale nature of the solar wind. Living Rev Sol Phys 16(1): 5. https://doi.org/ 10.1007/s41116-019-0021-0.

Viljanen A, Pirjola R, Pracser E, Katkalov J, Wik M. 2014. Geomagnetically induced currents in Europe - Modelled occurrence in a continent-wide power grid. J Space Weather Space Clim 4: A09. https://doi.org/10.1051/swsc/2014006.

Viljanen A, Tanskanen E, Pulkkinen A. 2006. Relation between substorm characteristics and rapid temporal variations of the ground magnetic field. Ann Geophys 24: 725-733. https://doi.org/ 10.5194/angeo-24-725-2006.

Výbošt'oková T, Švanda M. 2019. Statistical analysis of the correlation between anomalies in the Czech electric power grid and geomagnetic activity. Space Weather 17: 1208-1218. https://doi.org/10.1029/2019SW002181.

Wang K-R, Liu L-G, Li Y. 2015. Preliminary analysis on the interplanetary cause of geomagnetically induced current and its effect on power systems. Chinese Astron Astrophys 39: 78-88. https://doi.org/10.1016/j.chinastron.2015.01.003.

Watari S, Kunitake M, Kitamura K, Hori T, Kikuchi T, et al. 2009. Measurements of geomagnetically induced current in a power grid in Hokkaido, Japan. Space Weather 7: 03002. https://doi.org/ 10.1029/2008SW000417.

Zhang JJ, Wang C, Sun T, Liu YD. 2016. Risk assessment of the extreme interplanetary shock of 23 July 2012 on low-latitude power networks. Space Weather 14: 259-270. https://doi.org/ 10.1002/2015SW001347.

Zois IP. 2013. Solar activity and transformer failures in the Greek national electric grid. J Space Weather Space Clim 3: A32. https://doi.org/10.1051/swsc/2013055. 


\section{Appendix}

\section{Complete set of figures for all distributors}
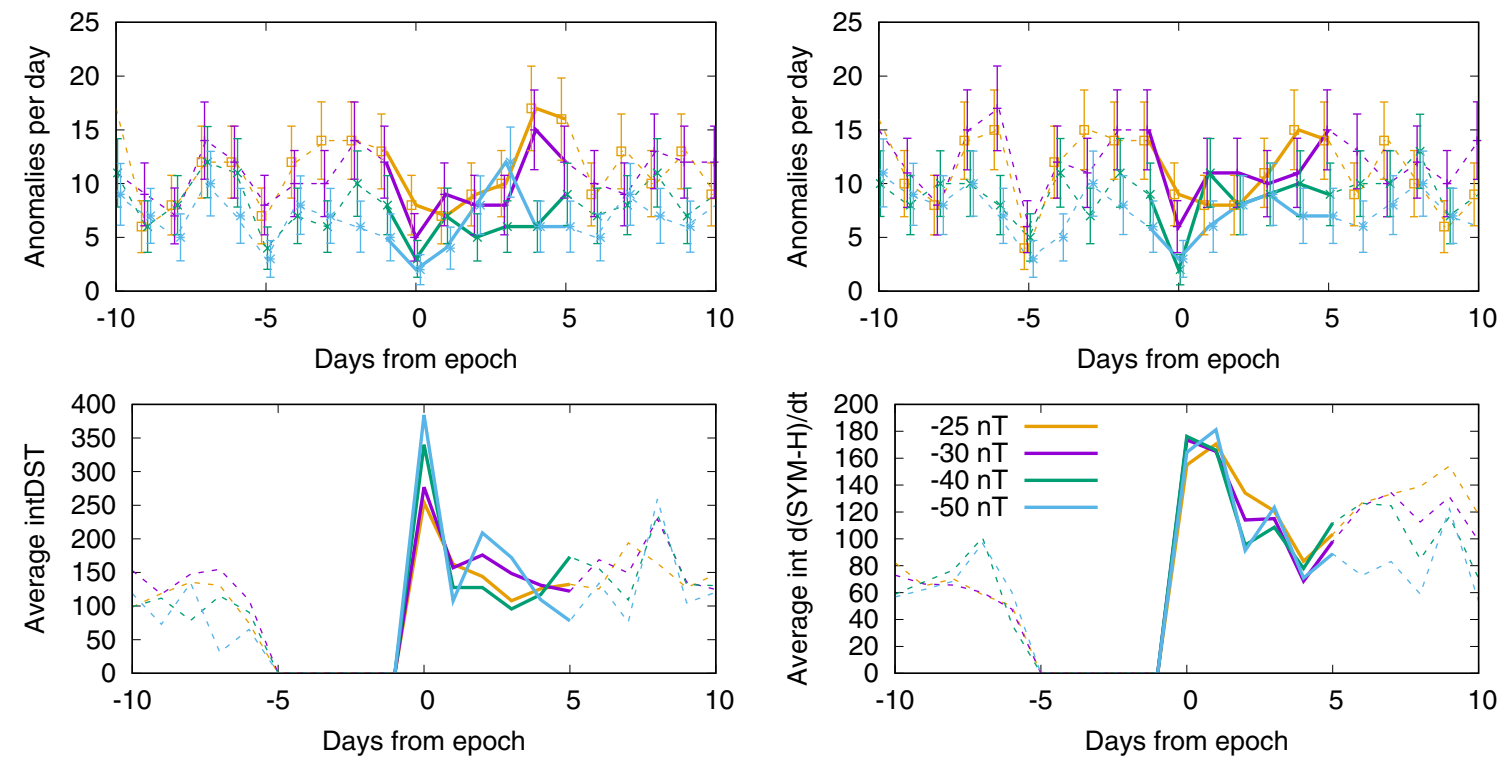

Fig. A.1. Plot of epoch-superposed D1 series considering IntDst with different thresholds (left) and Int(d(SYM-H)/dt) (right). The solid lines indicate the superposed anomaly rates (upper row) or reference time series (lower row) in the Days -1 to +5 from the epoch, whereas the dashed lines represent the same quantity for the remaining days.
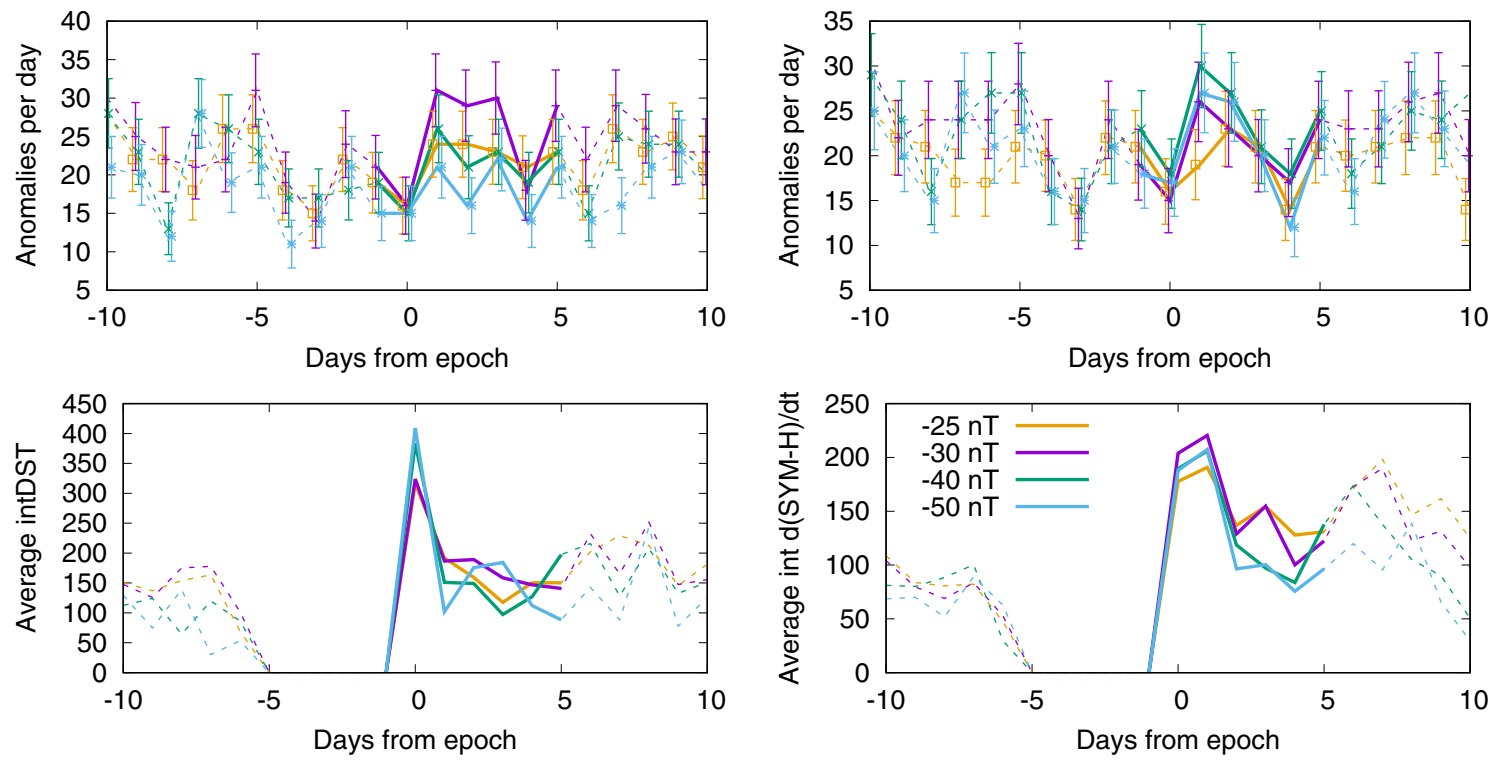

Fig. A.2. Same as Figure A.1 only for series D2. 
M. Švanda et al.: J. Space Weather Space Clim. 2020, 10, 26
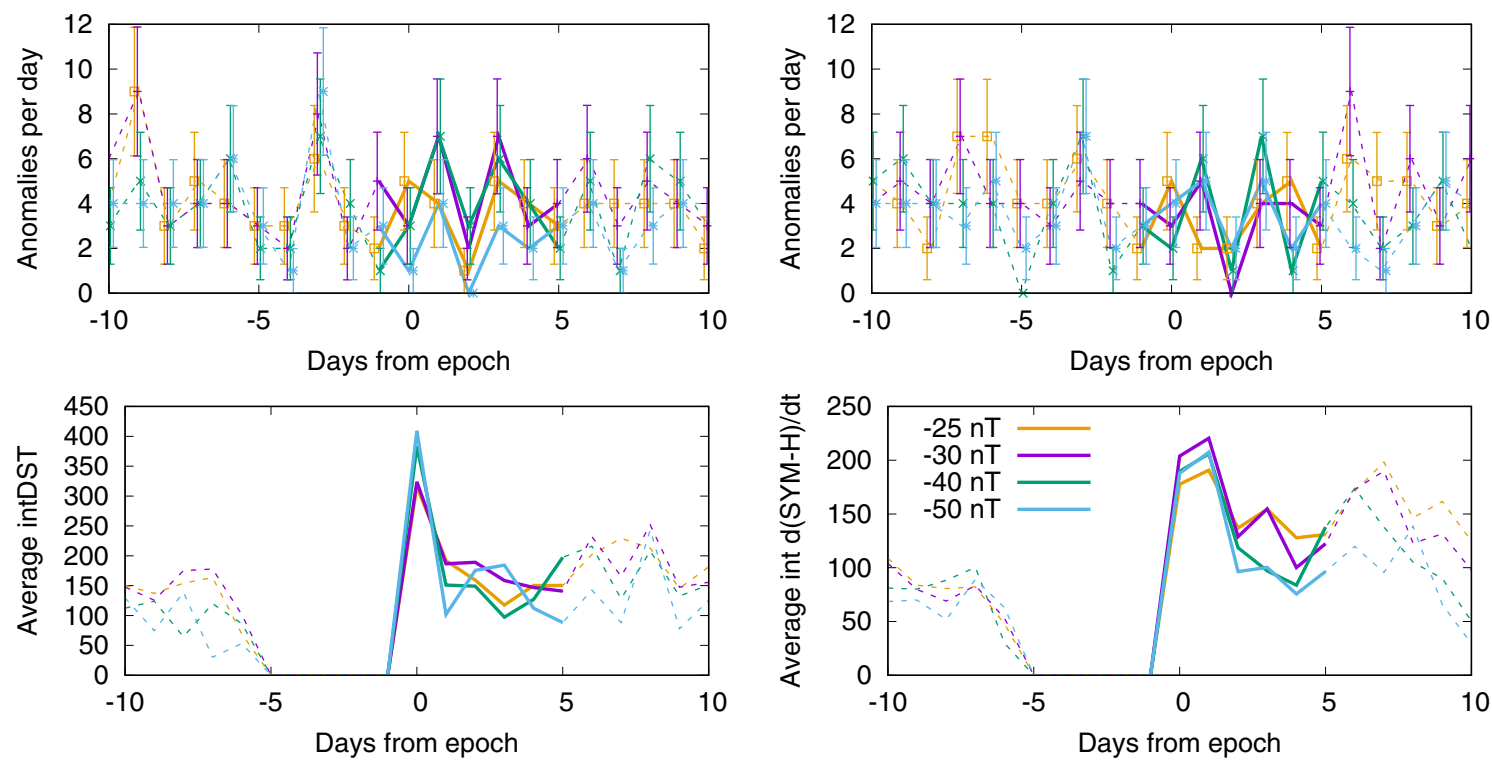

Fig. A.3. Same as Figure A.1 only for series D3.
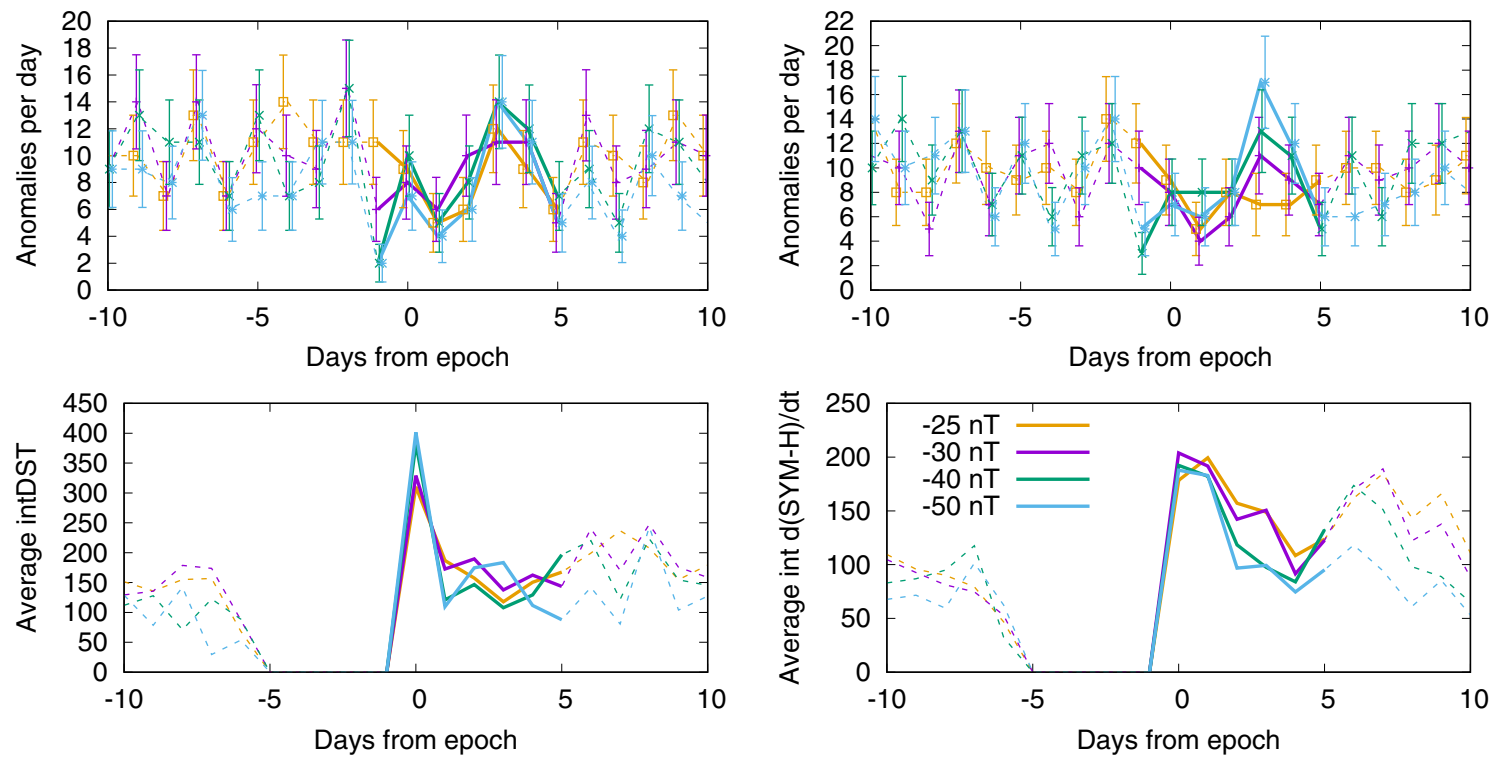

Fig. A.4. Same as Figure A.1 only for series D4. 
M. Švanda et al.: J. Space Weather Space Clim. 2020, 10, 26
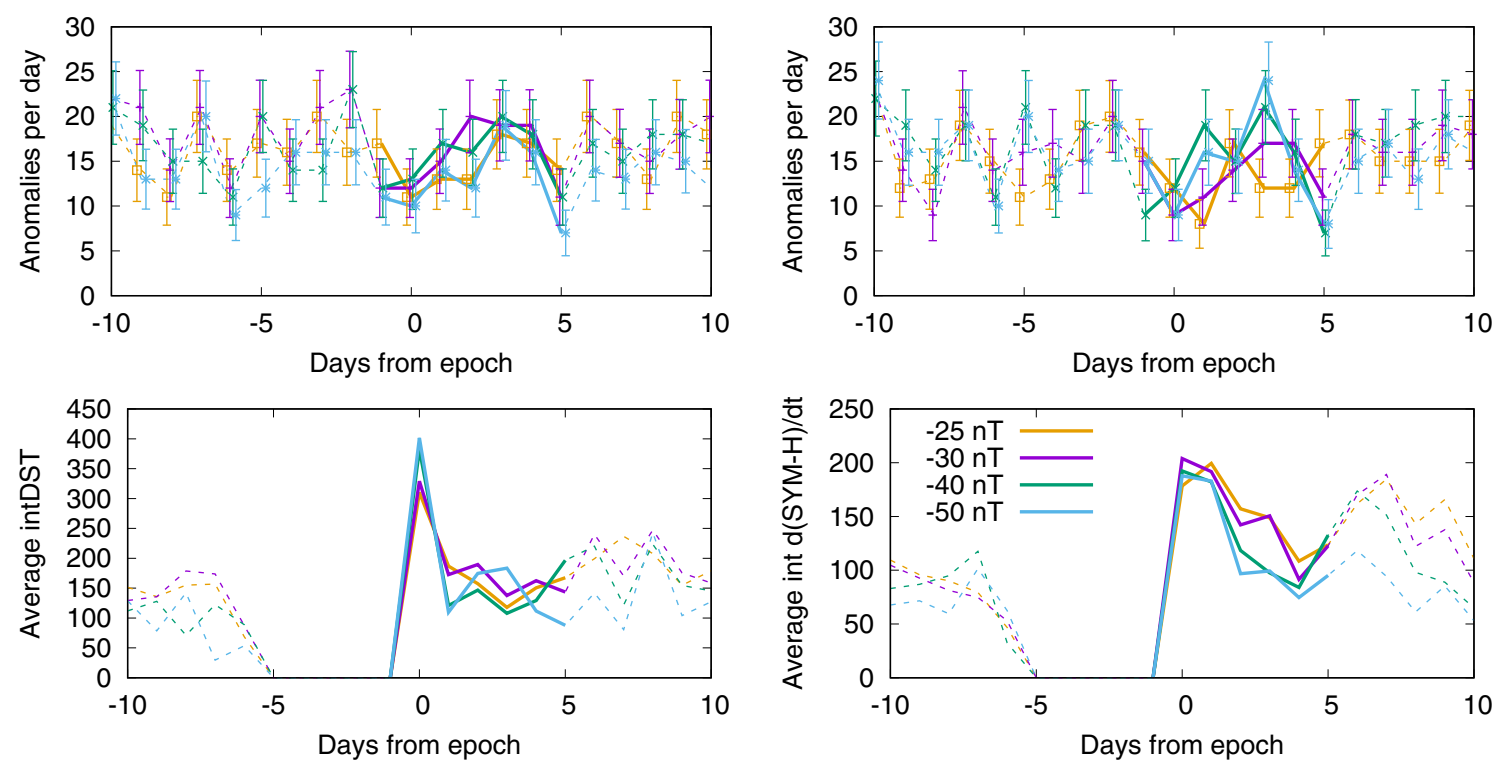

Fig. A.5. Same as Figure A.1 only for series D5.
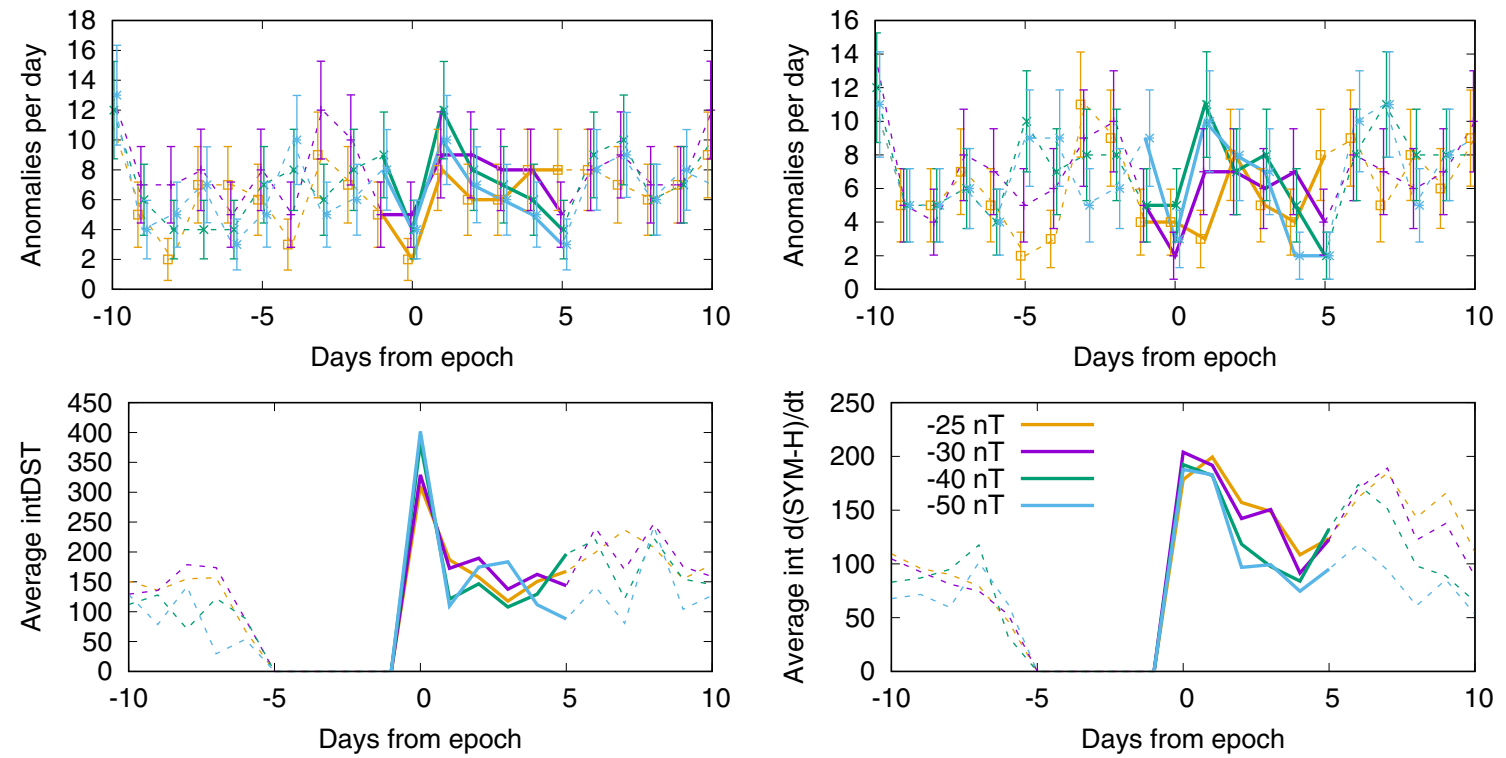

Fig. A.6. Same as Figure A.1 only for series D6. 
M. Švanda et al.: J. Space Weather Space Clim. 2020, 10, 26
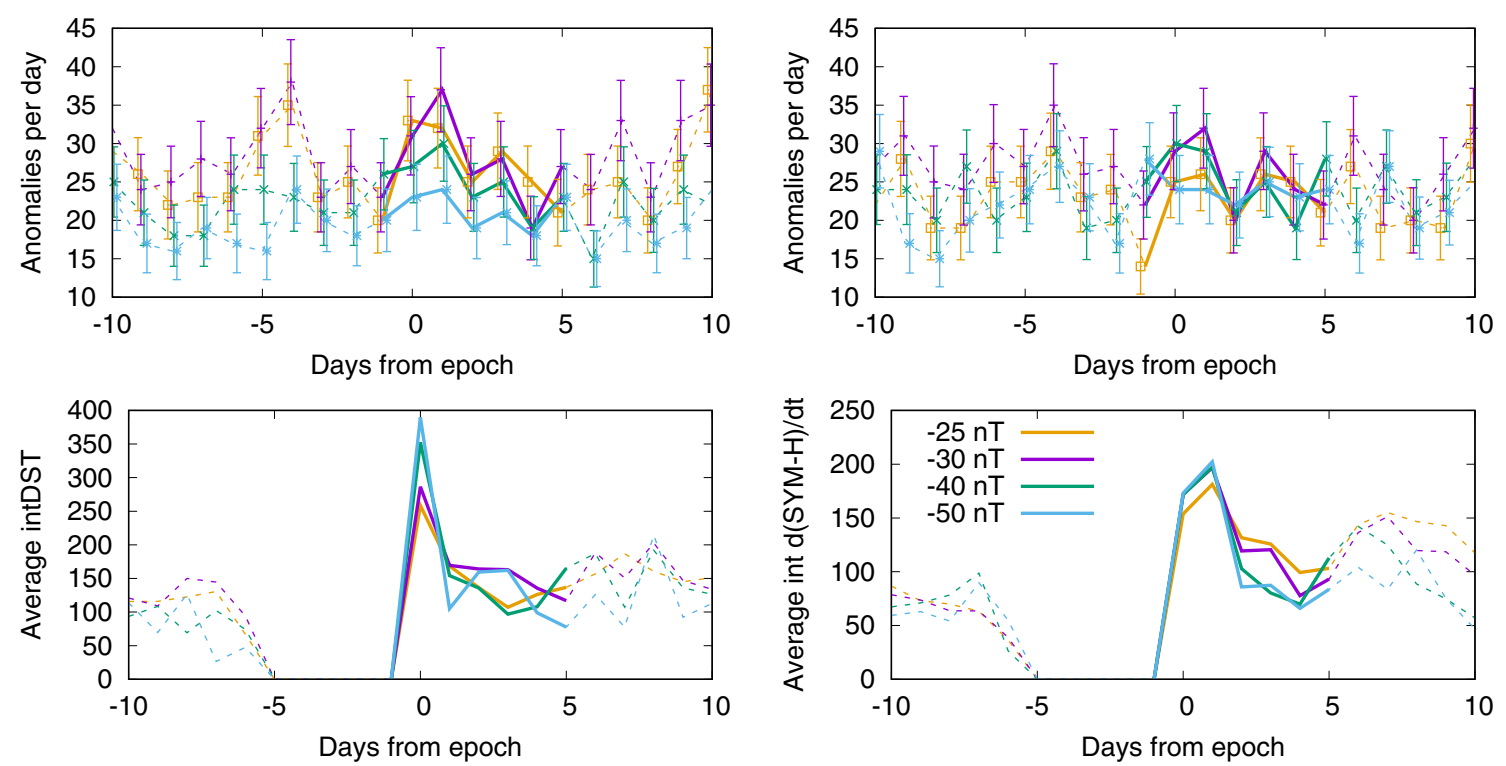

Fig. A.7. Same as Figure A.1 only for series D7.
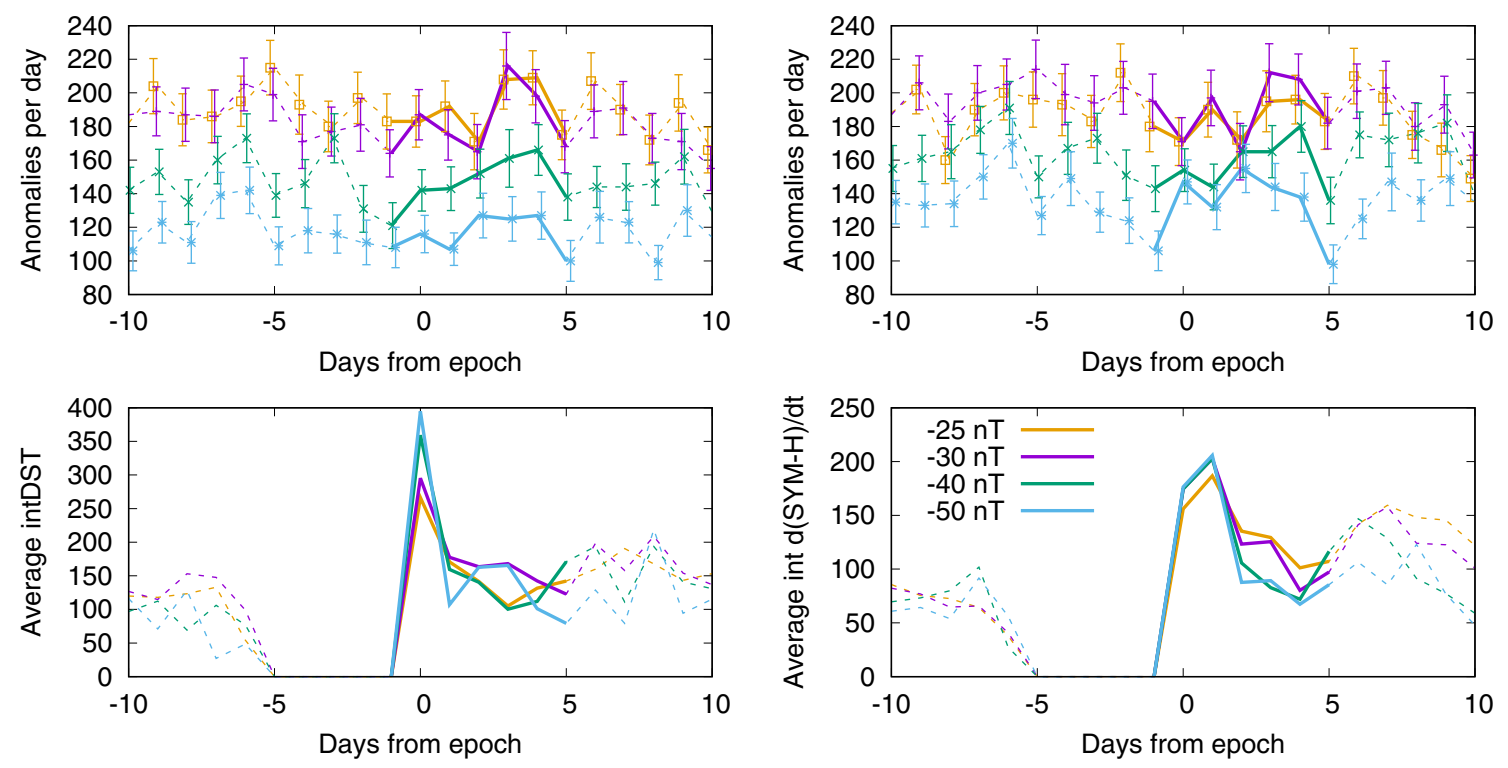

Fig. A.8. Same as Figure A.1 only for series D8. 
M. Švanda et al.: J. Space Weather Space Clim. 2020, 10, 26
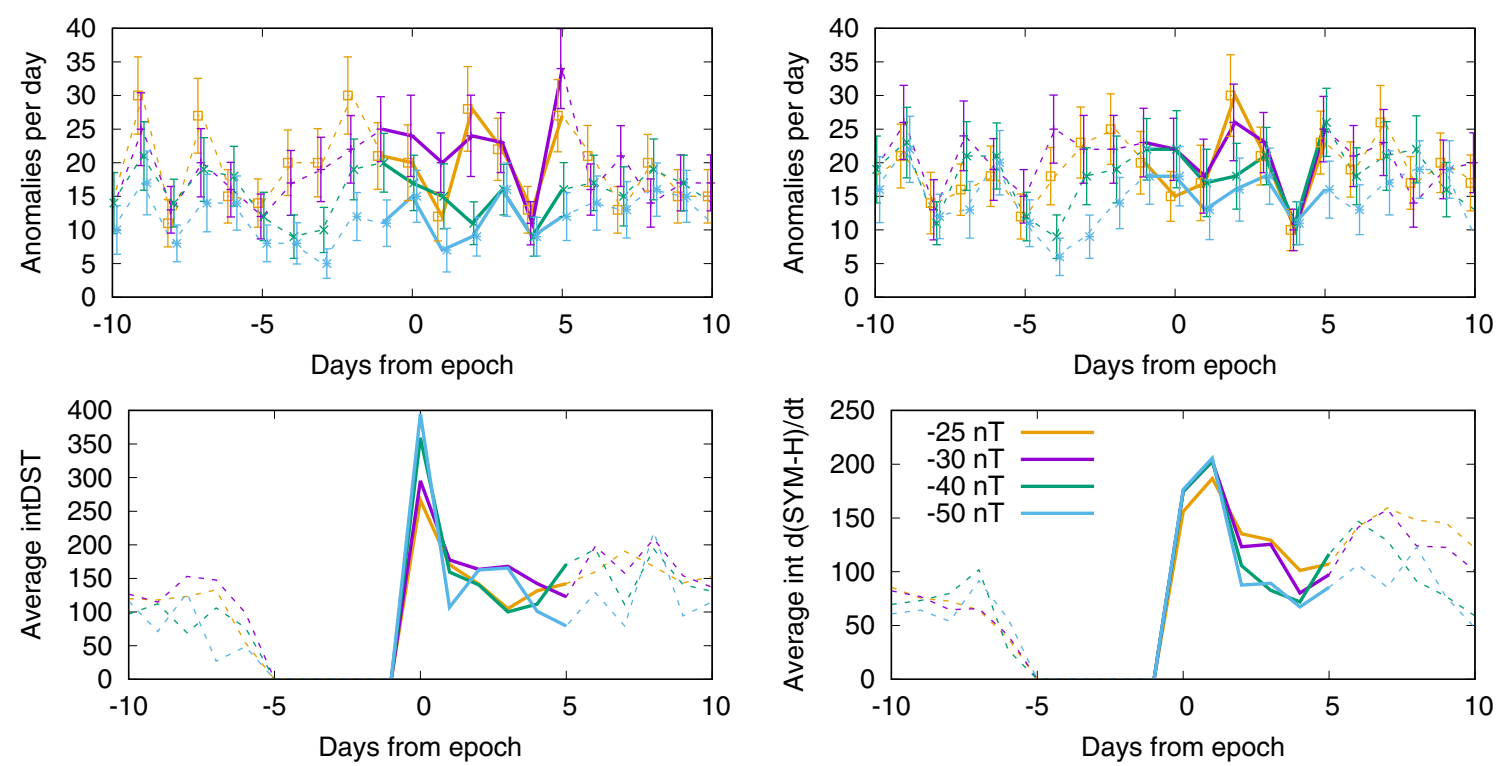

Fig. A.9. Same as Figure A.1 only for series D9.
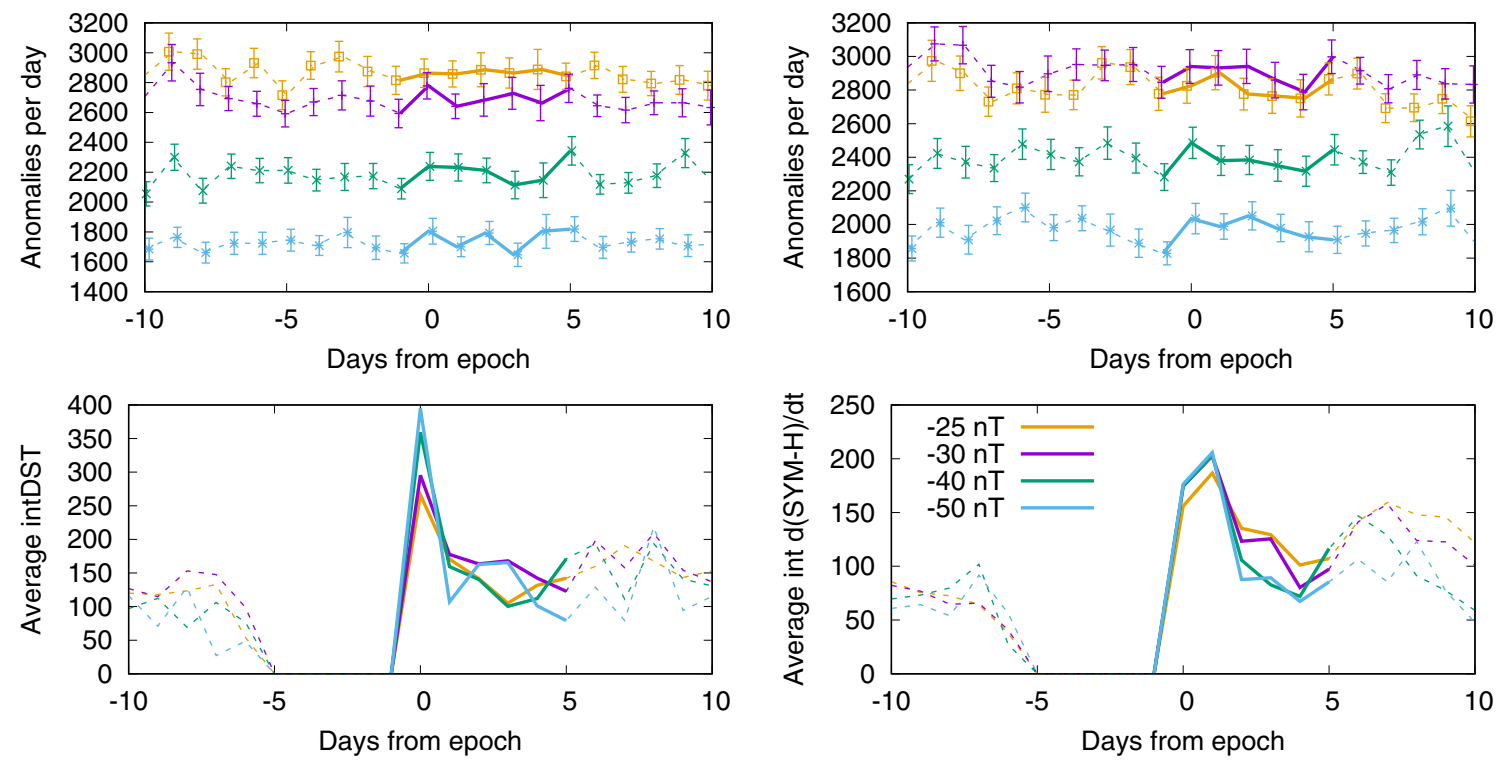

Fig. A.10. Same as Figure A.1 only for series D10. 
M. Švanda et al.: J. Space Weather Space Clim. 2020, 10, 26
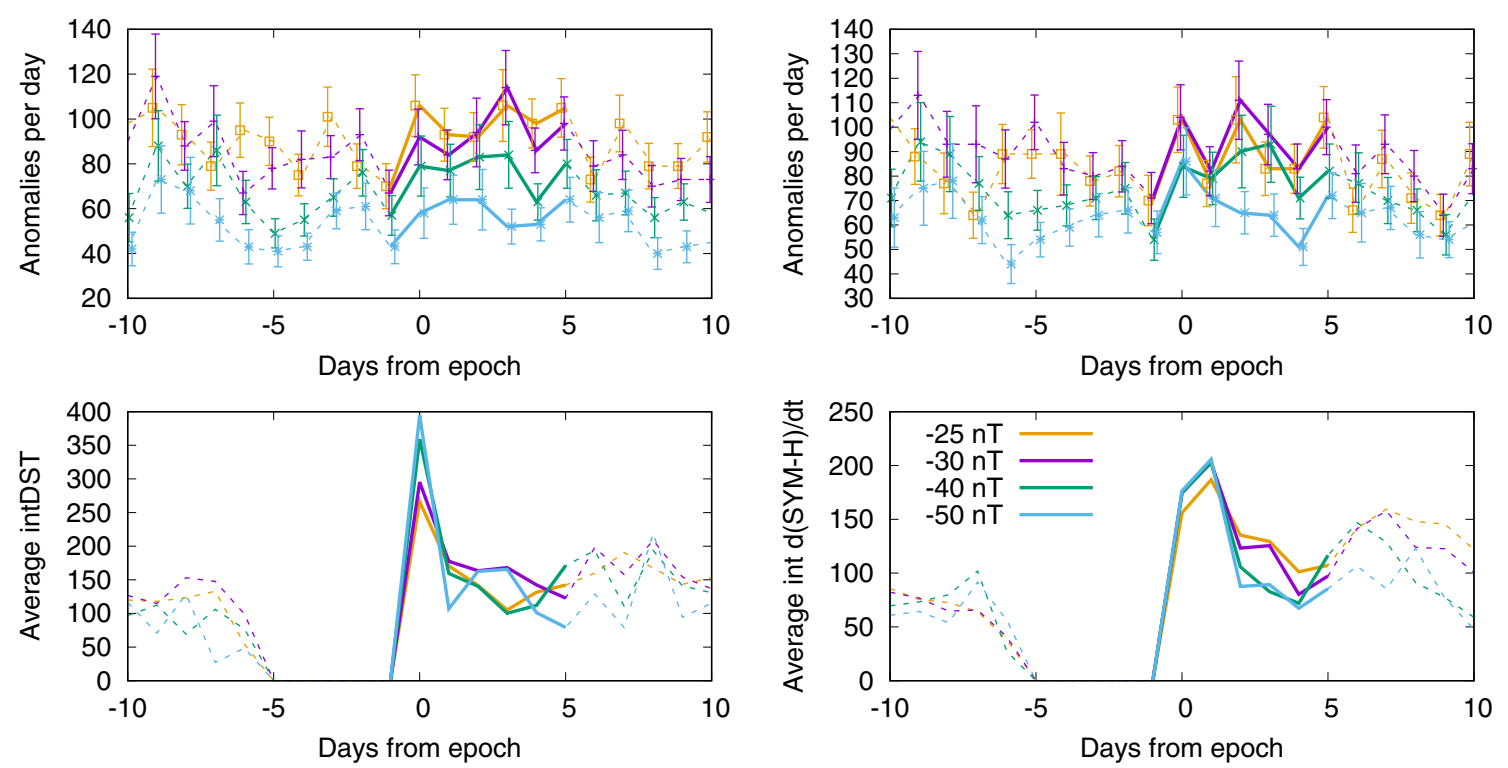

Fig. A.11. Same as Figure A.1 only for series D11.
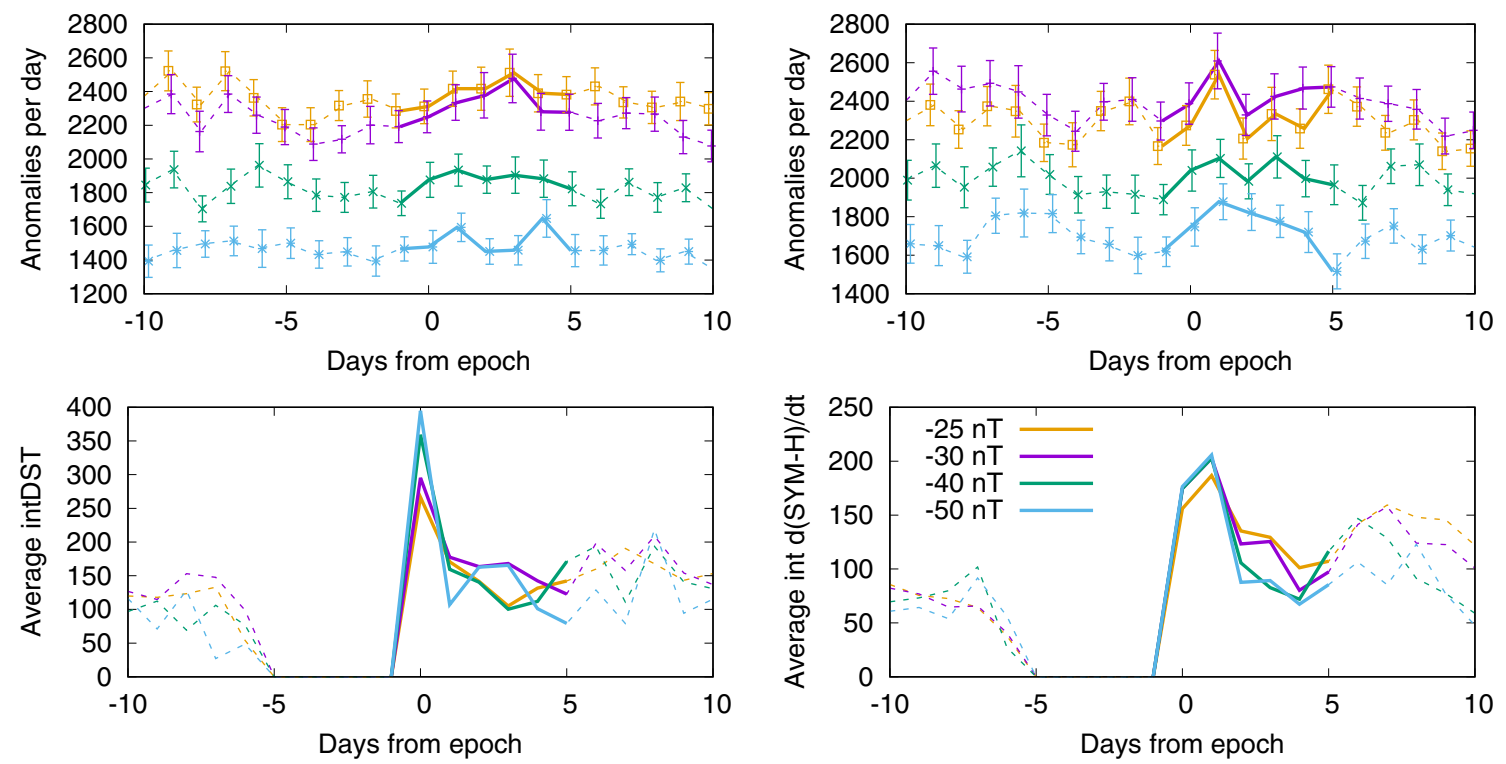

Fig. A.12. Same as Figure A.1 only for series D12. 

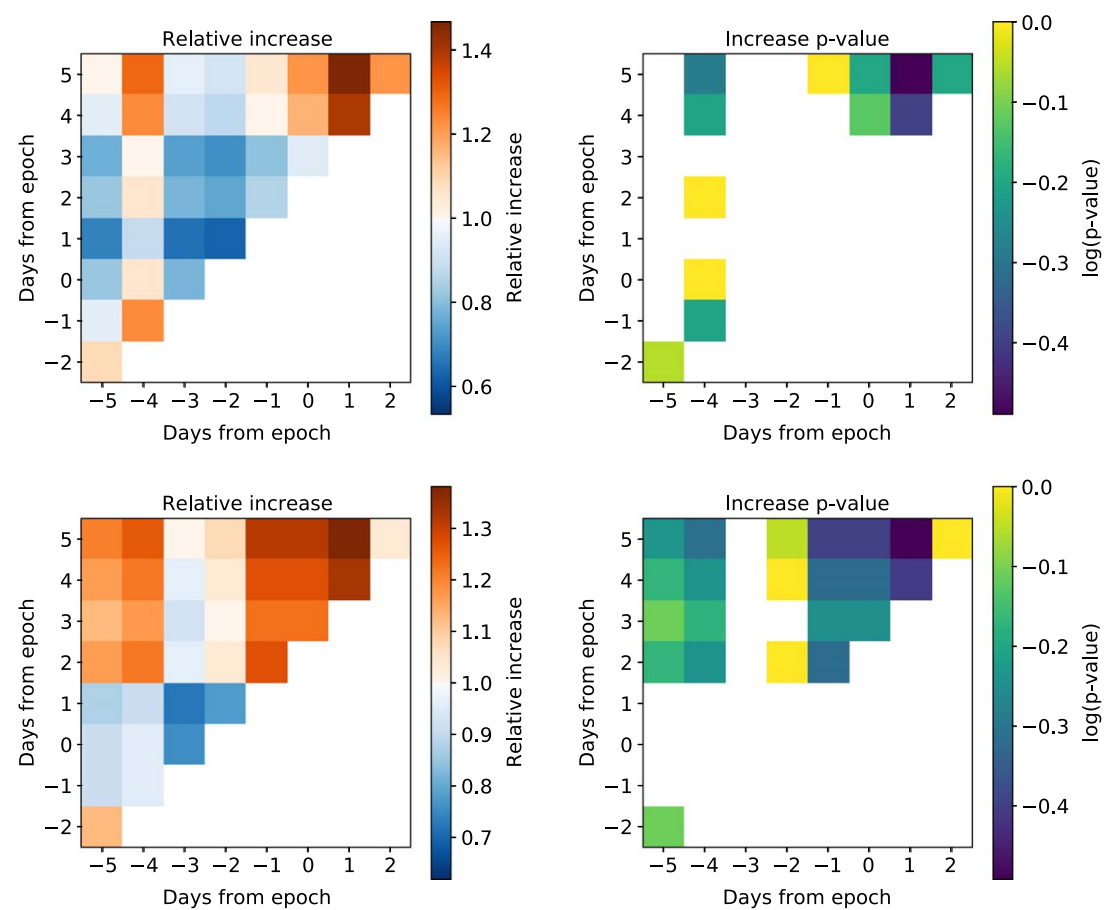

Fig. A.13. The map for D1 of increases (or decreases) of the number of anomalies as a function of the middle day of the first and second 3-day periods, together with maps of the corresponding $p$-values. The upper row is computed for IntDst as the reference series, whereas the lower row is for $\operatorname{Int}(\mathrm{d}(\mathrm{SYM}-\mathrm{H})=\mathrm{d} t)$ series. The $p$-values in the right column are evaluated only if there is an increase of the grid anomaly rates in the second 3-day period as compared to the first 3-day period. Note the logarithmic scale of the plotted $p$-values: $p=0.0055$ (the adopted level of statistical significance for individual bins) corresponds to $\log p=-2.26$. Statistically significant bins are indicated by white dots. Blank bins are indicated by the white colour.
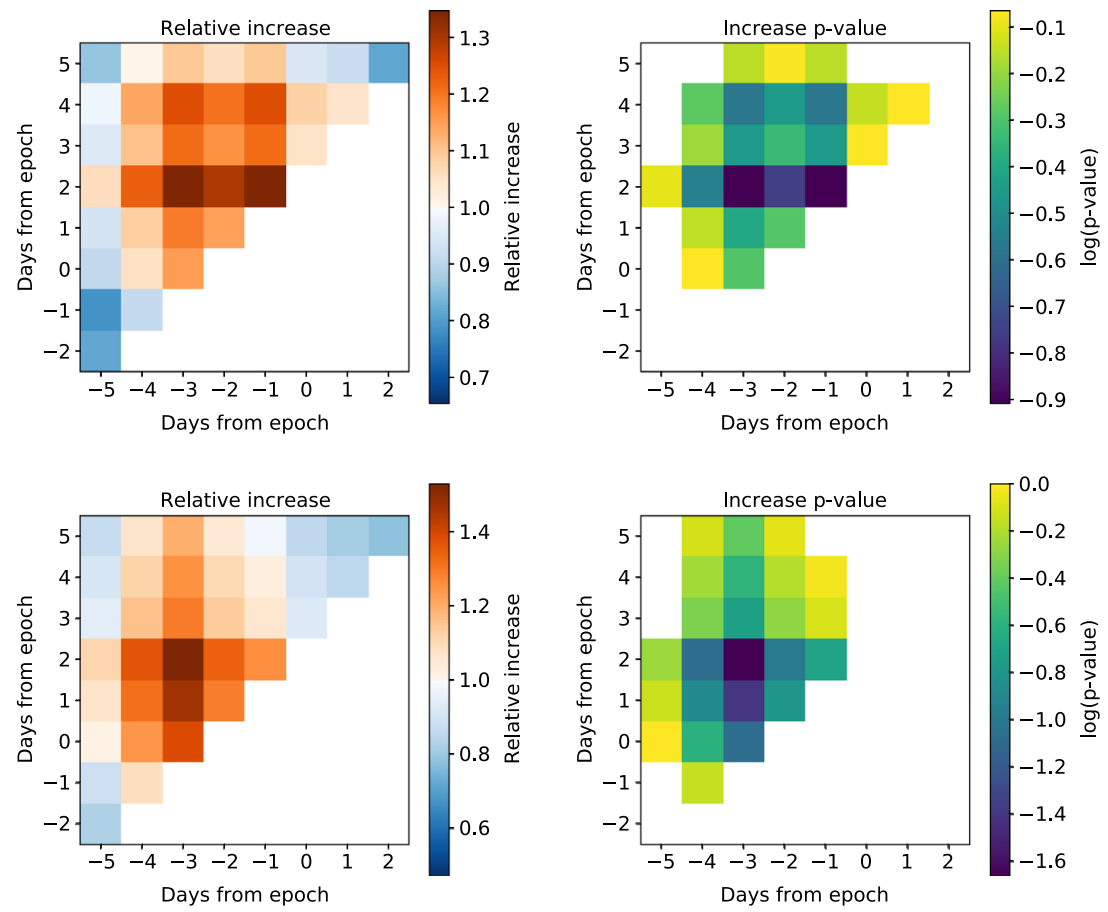

Fig. A.14. Same as Figure A.13 only for series D2. 

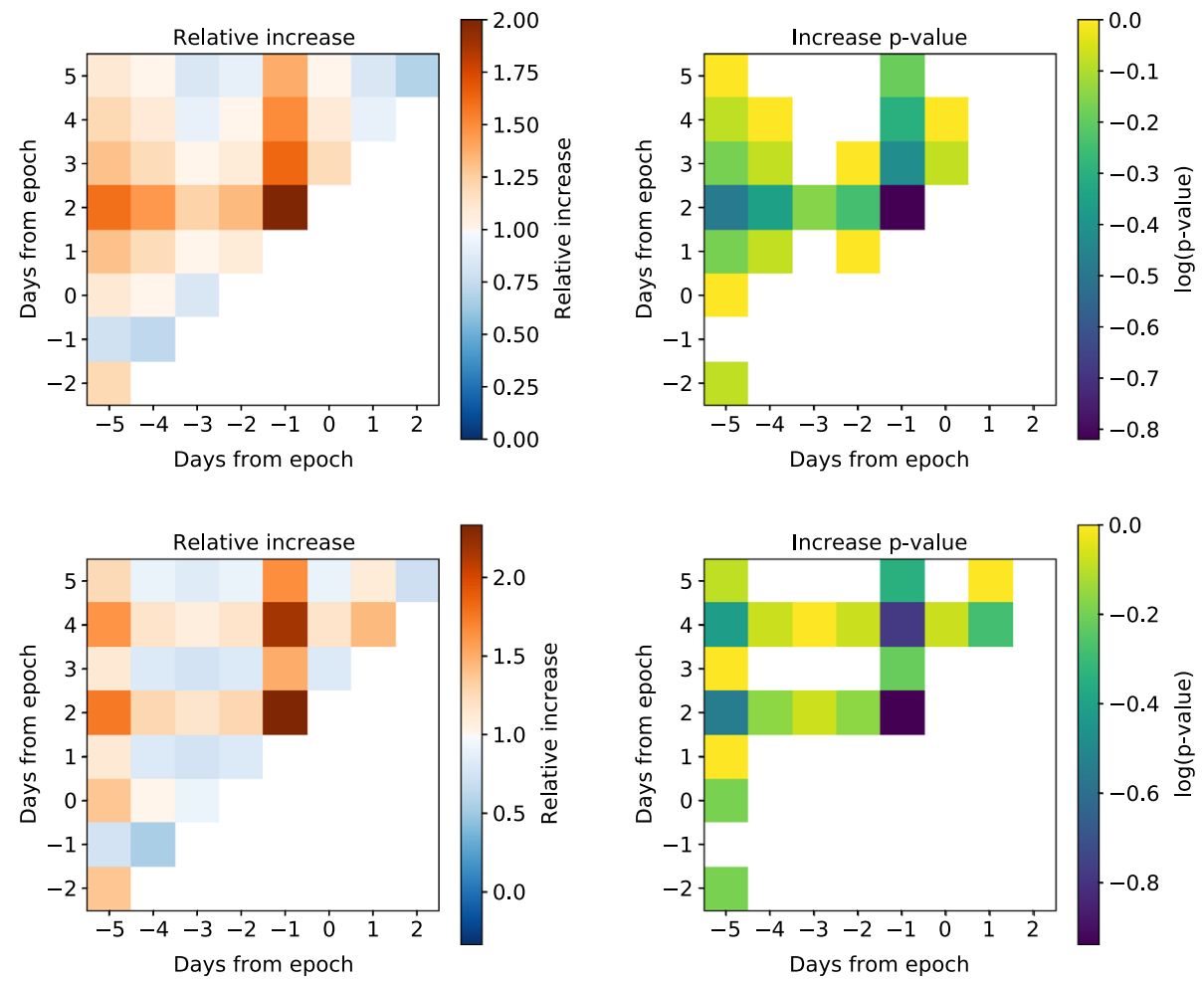

Fig. A.15. Same as Figure A.13 only for series D3.
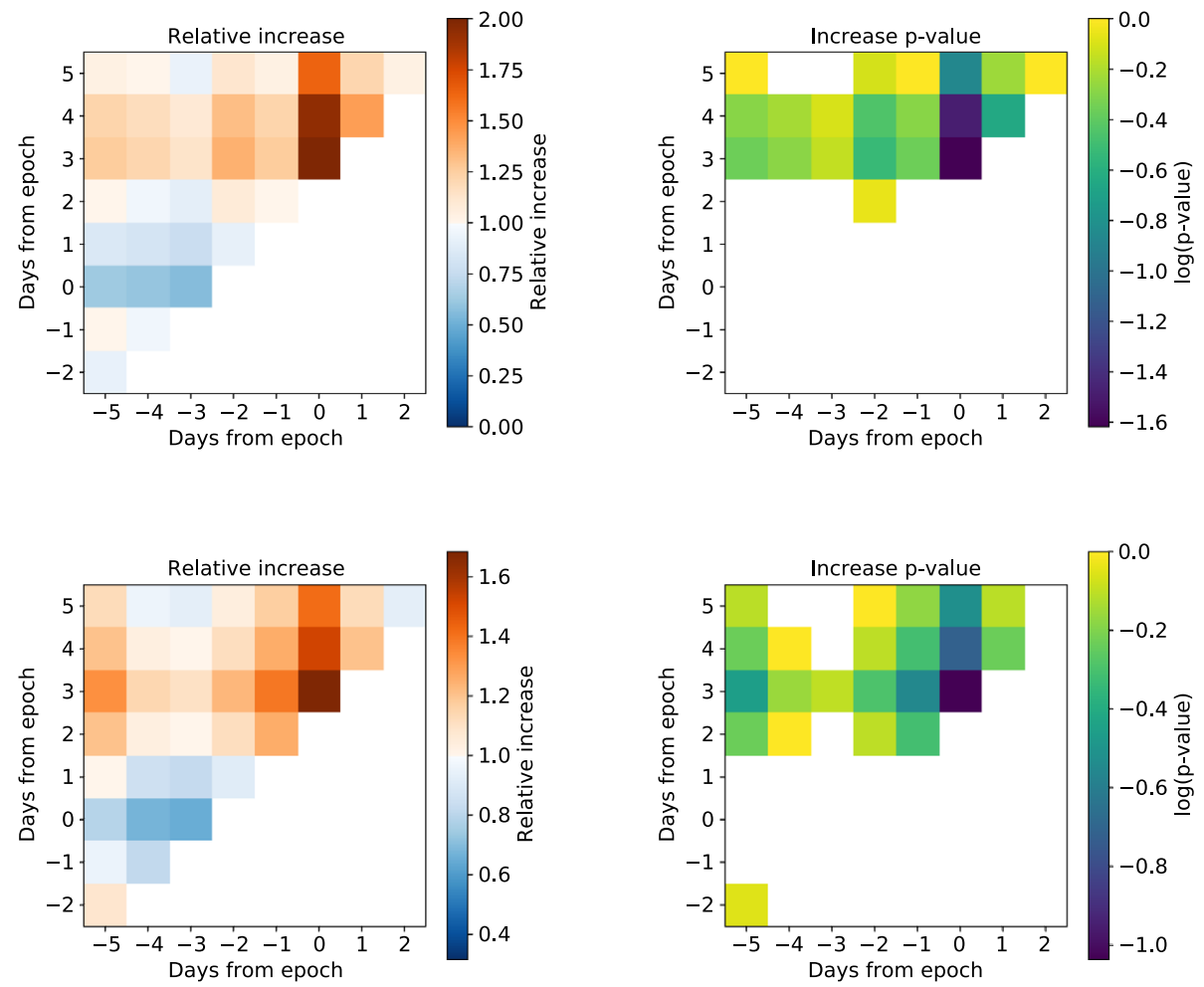

Fig. A.16. Same as Figure A.13 only for series D4. 

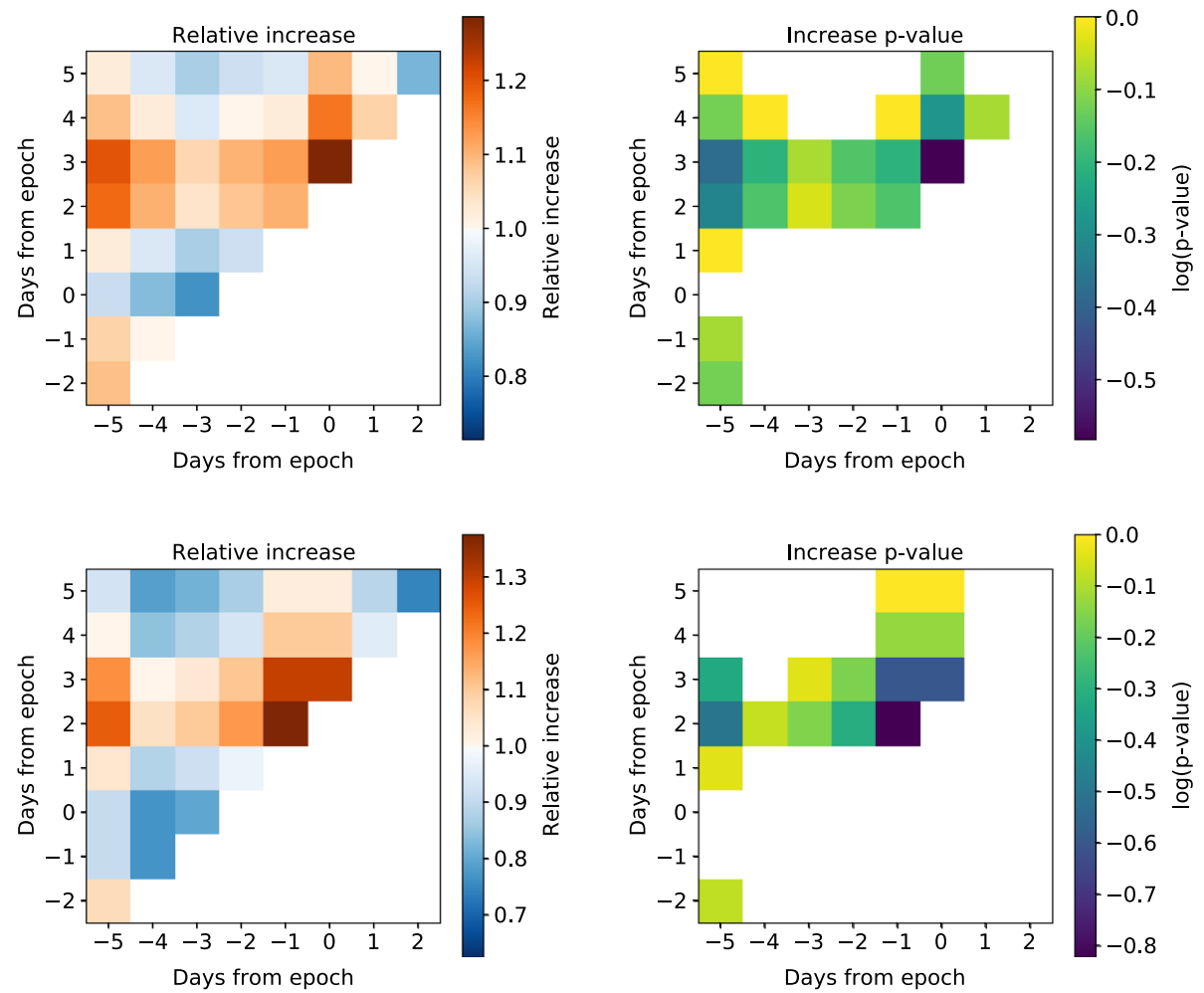

Fig. A.17. Same as Figure A.13 only for series D5.
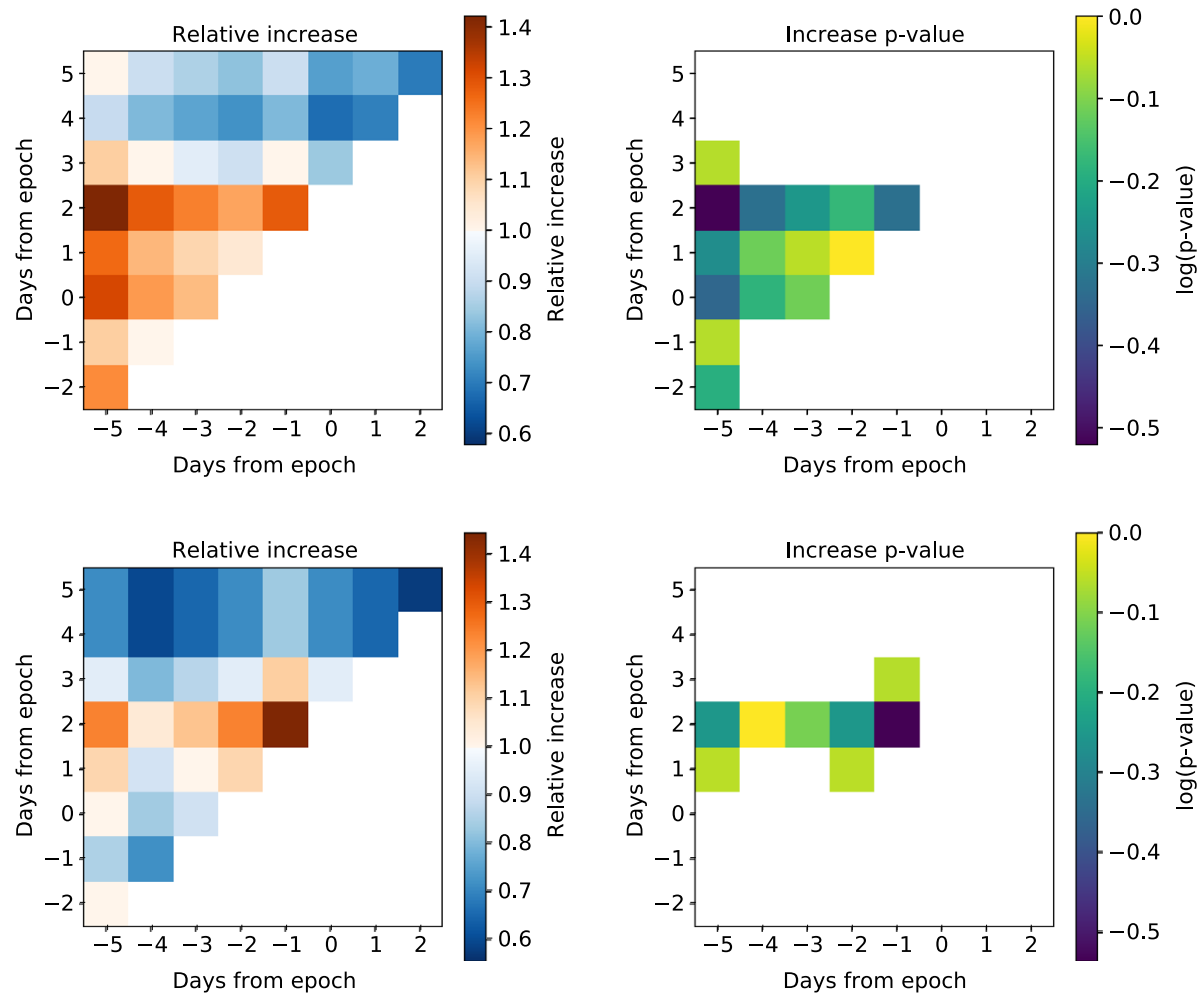

Fig. A.18. Same as Figure A.13 only for series D6. 
M. Švanda et al.: J. Space Weather Space Clim. 2020, 10, 26
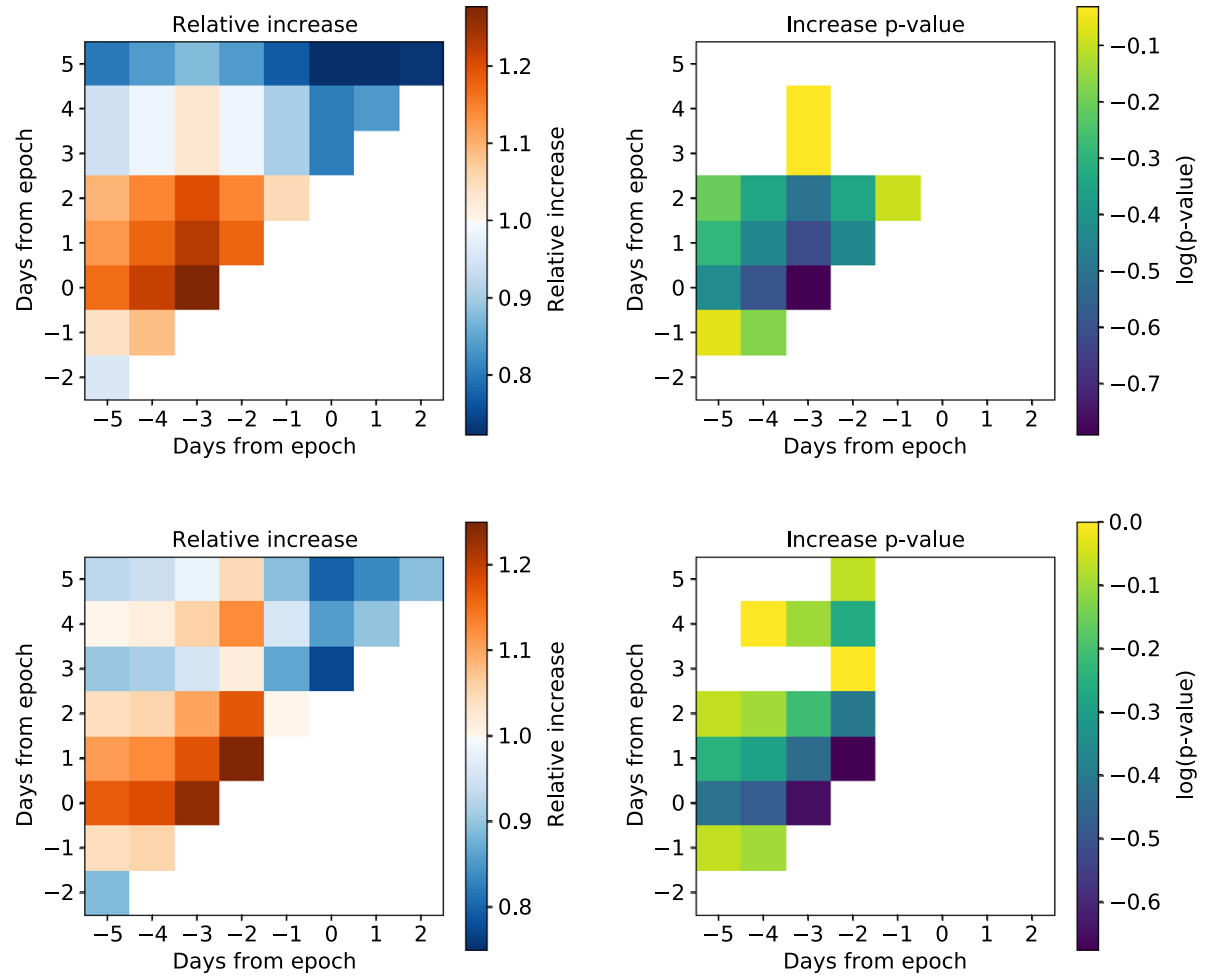

Fig. A.19. Same as Figure A.13 only for series D7.
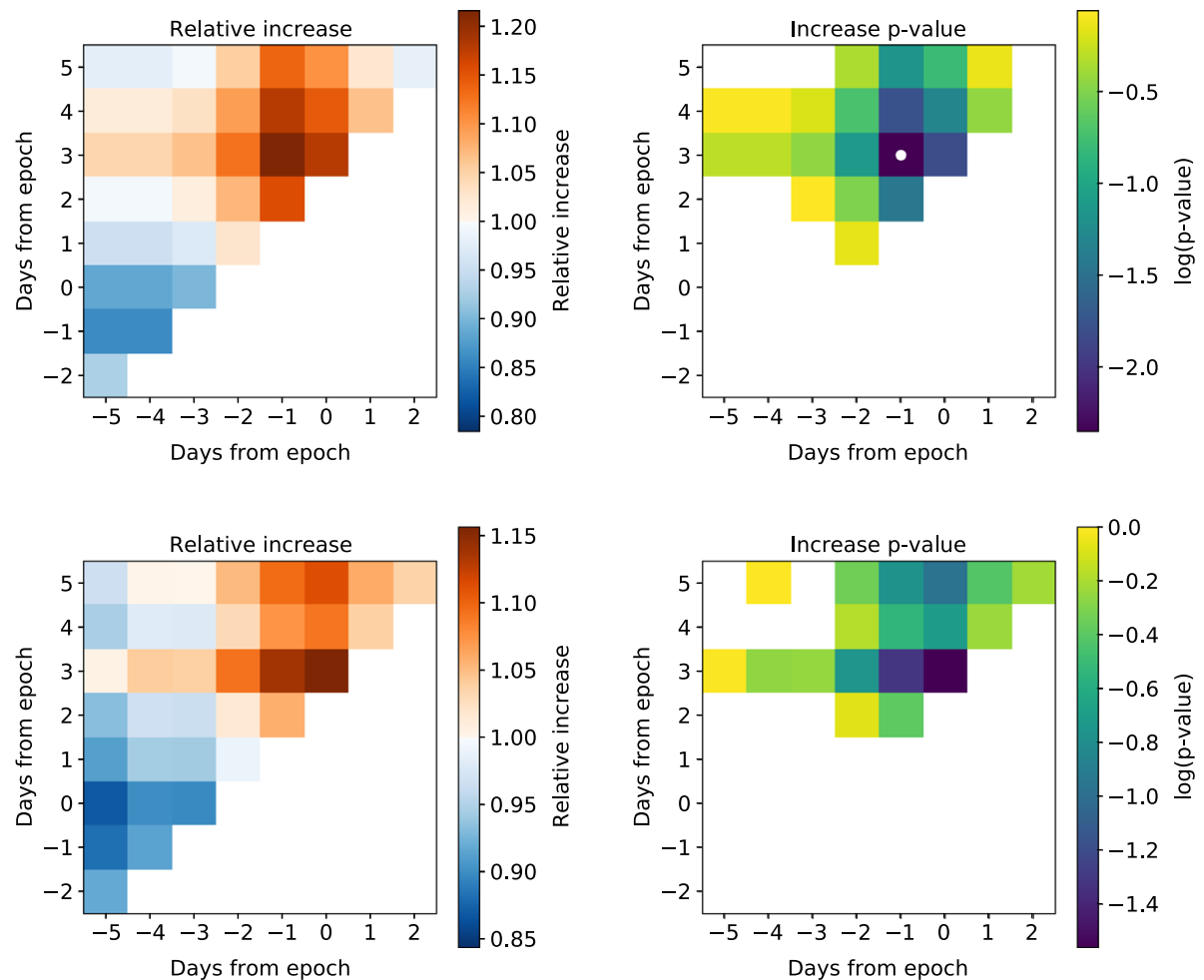

Fig. A.20. Same as Figure A.13 only for series D8. 

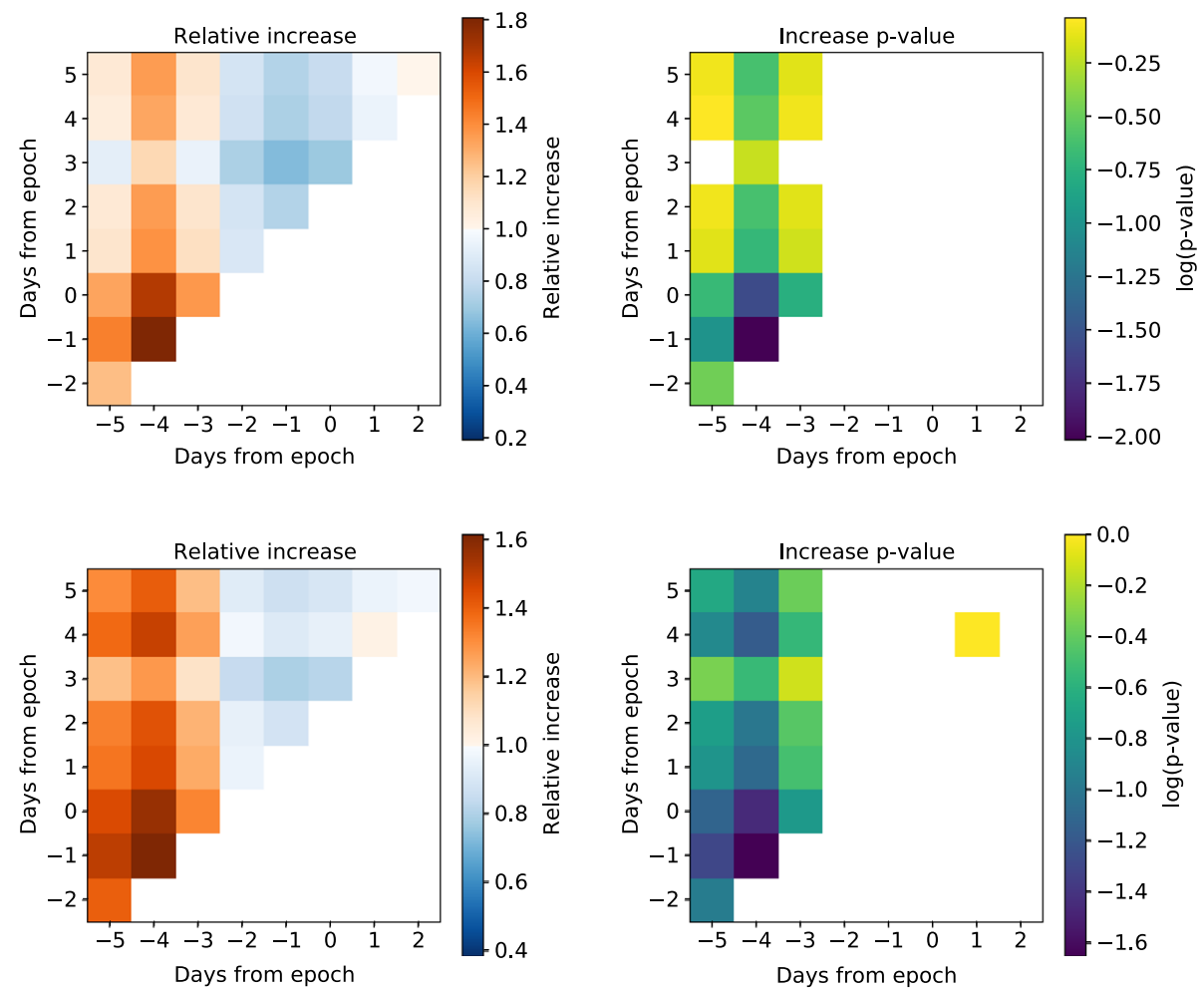

Fig. A.21. Same as Figure A.13 only for series D9.
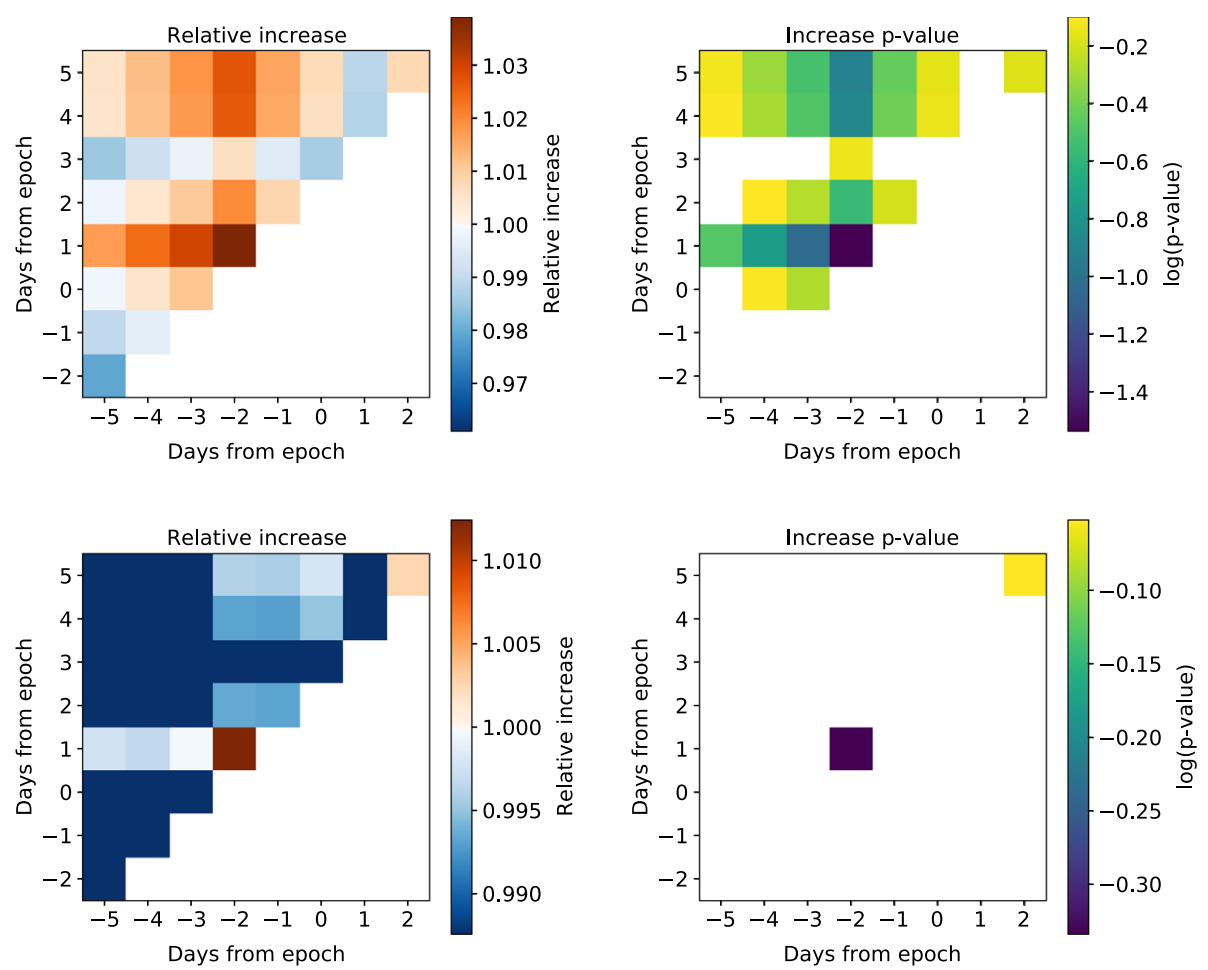

Fig. A.22. Same as Figure A.13 only for series D10. 

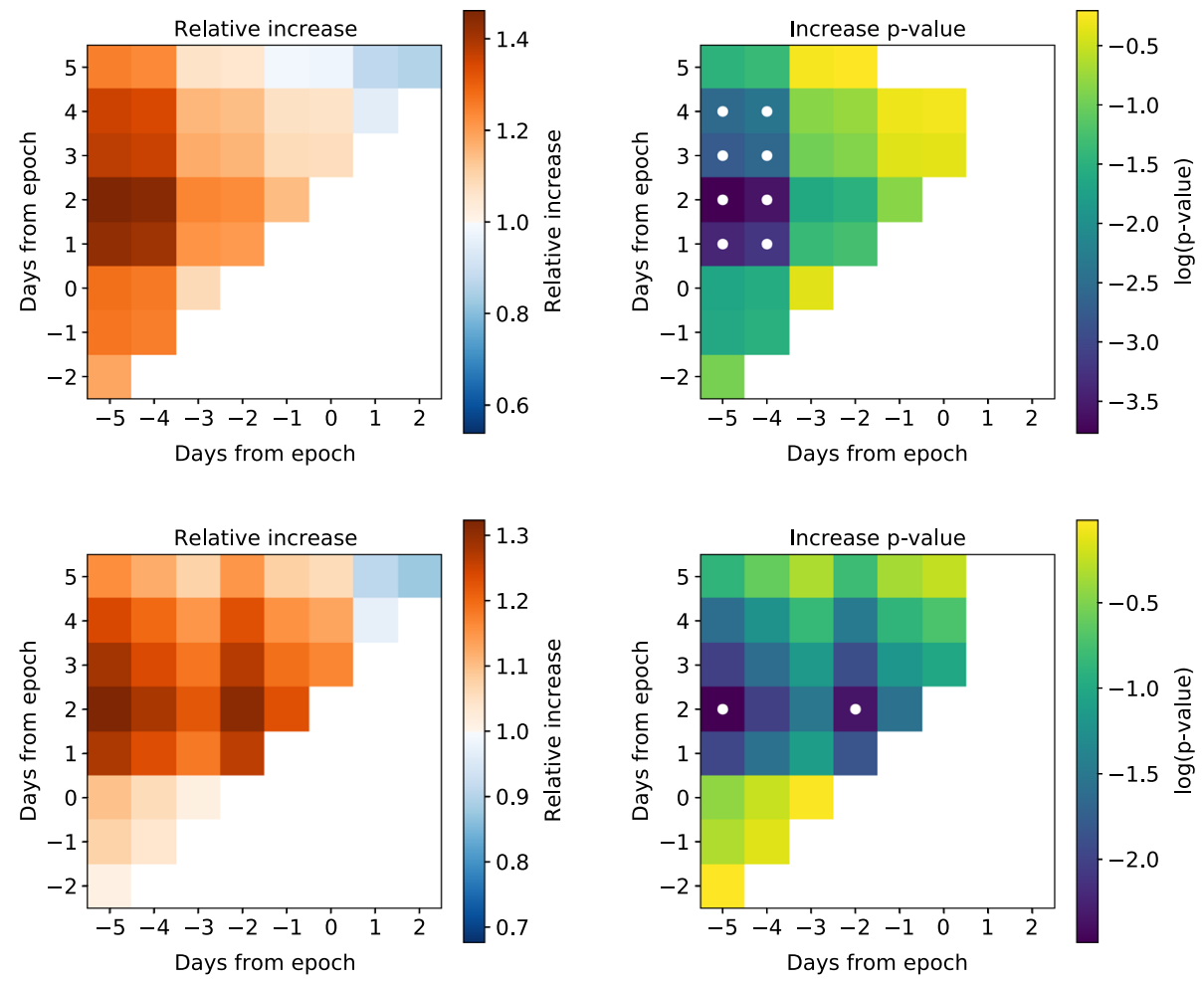

Fig. A.23. Same as Figure A.13 only for series D11.
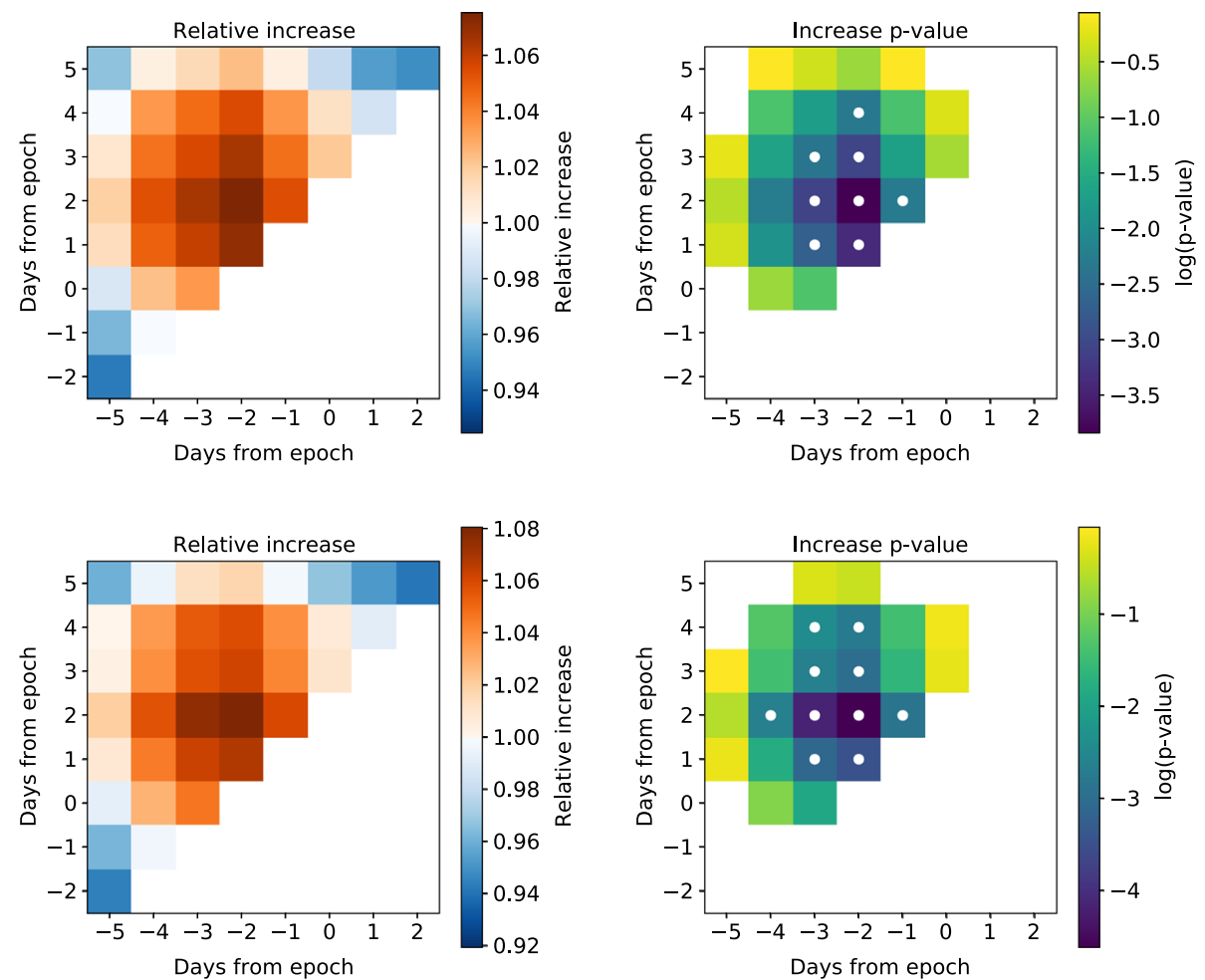

Fig. A.24. Same as Figure A.13 only for series D12. 

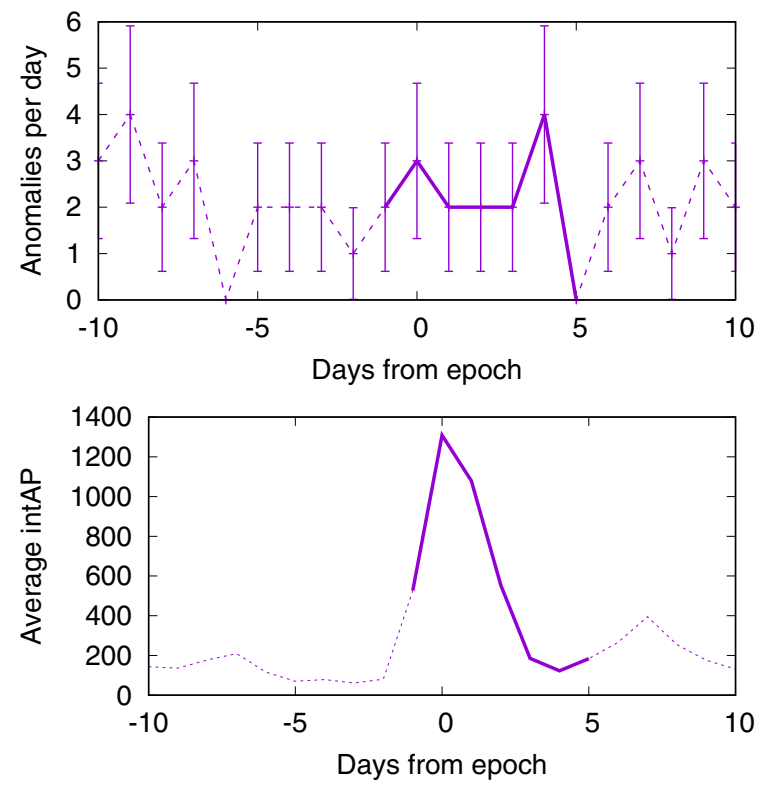

Fig. A.25. Plot of epoch-superposed D1 of increases (or decreases) of the daily number of anomalies as a function of time, considering the IntAp parameter. Solid lines indicate superposed daily rates of anomalies (upper row) or geomagnetic activity IntAp in the reference time series (bottom row) during Days -1 to +5 from the epoch (Day 0 ), whereas dashed lines show the same quantities for the remaining days.
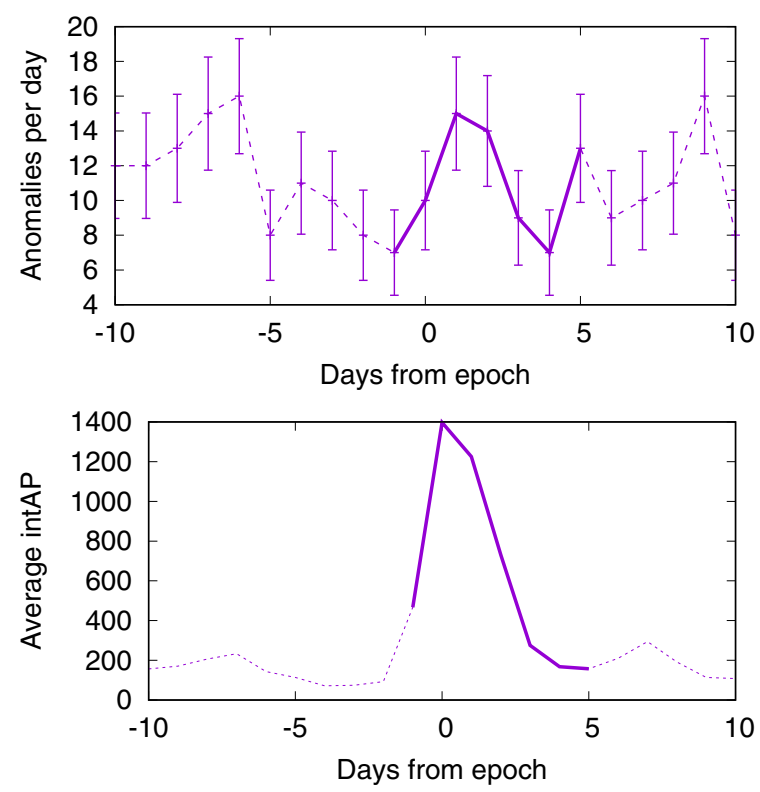

Fig. A.26. Same as Figure A.25 only for series D2.
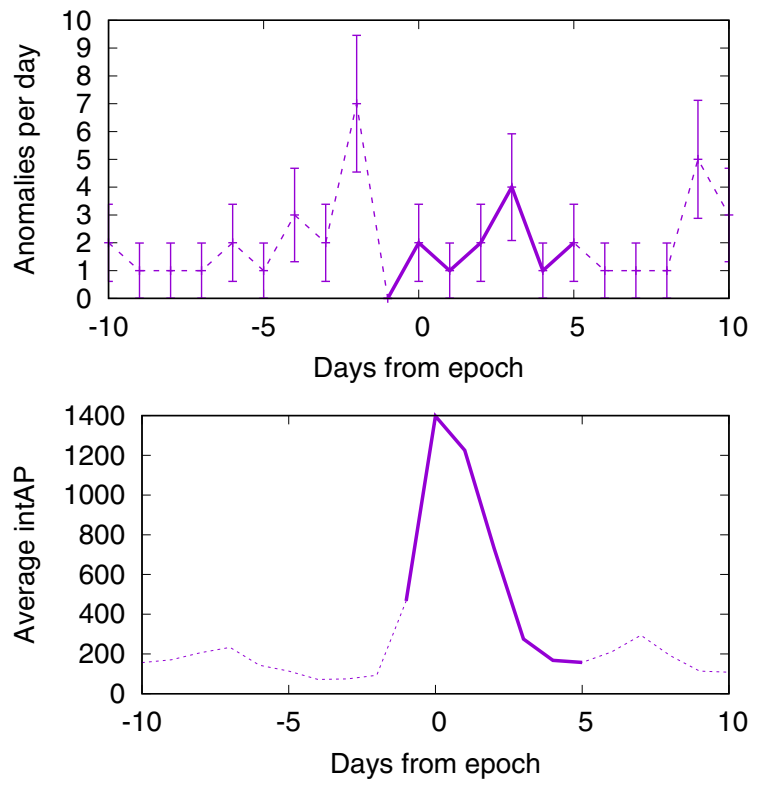

Fig. A.27. Same as Figure A.25 only for series D3.
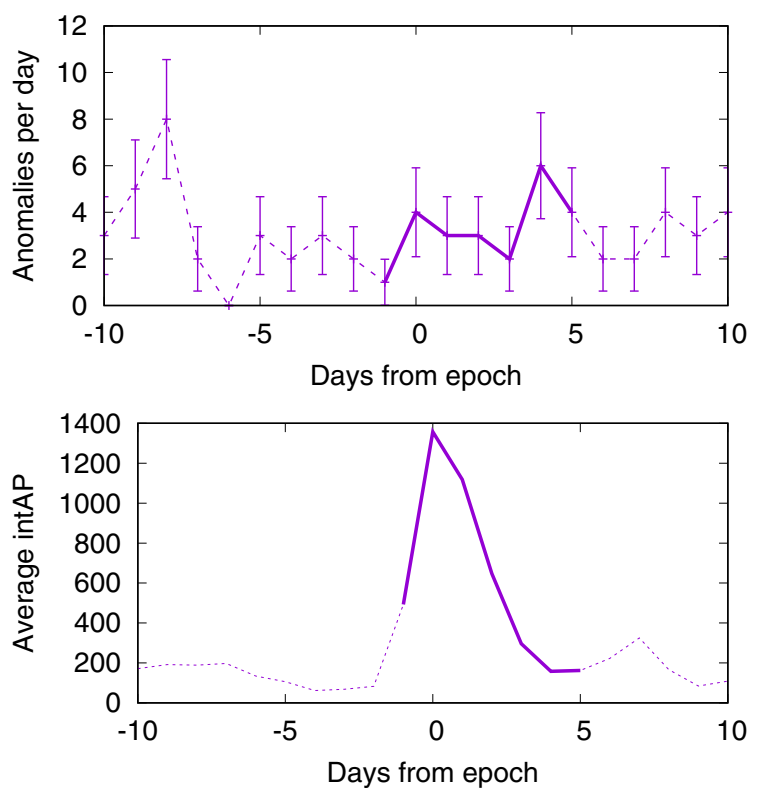

Fig. A.28. Same as Figure A.25 only for series D4. 

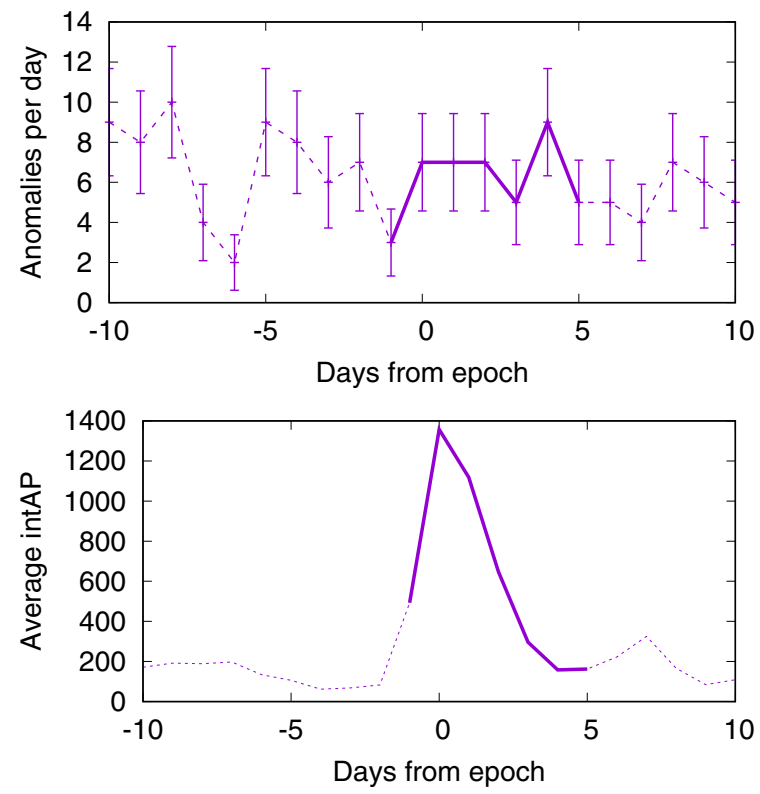

Fig. A.29. Same as Figure A.25 only for series D5.
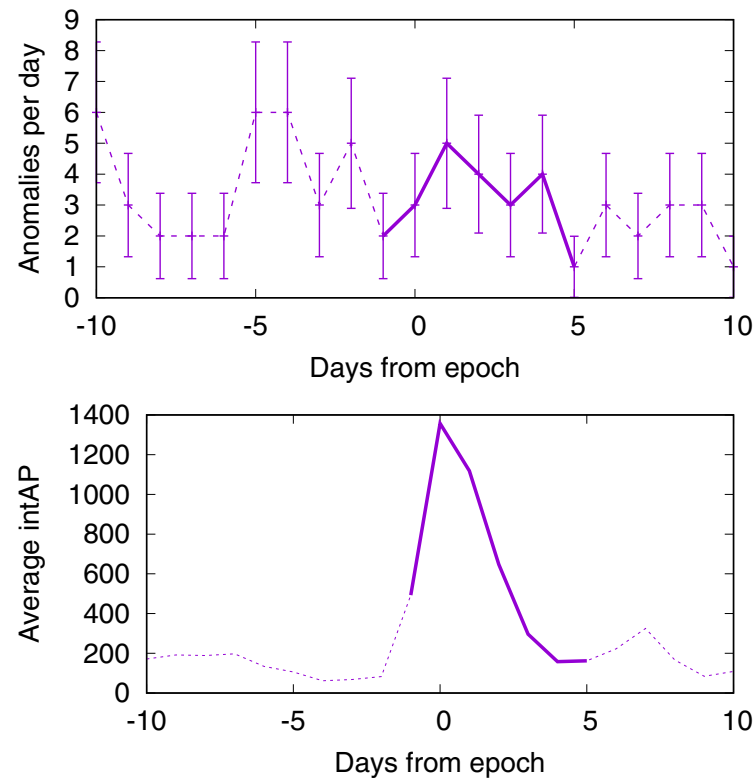

Fig. A.30. Same as Figure A.25 only for series D6.
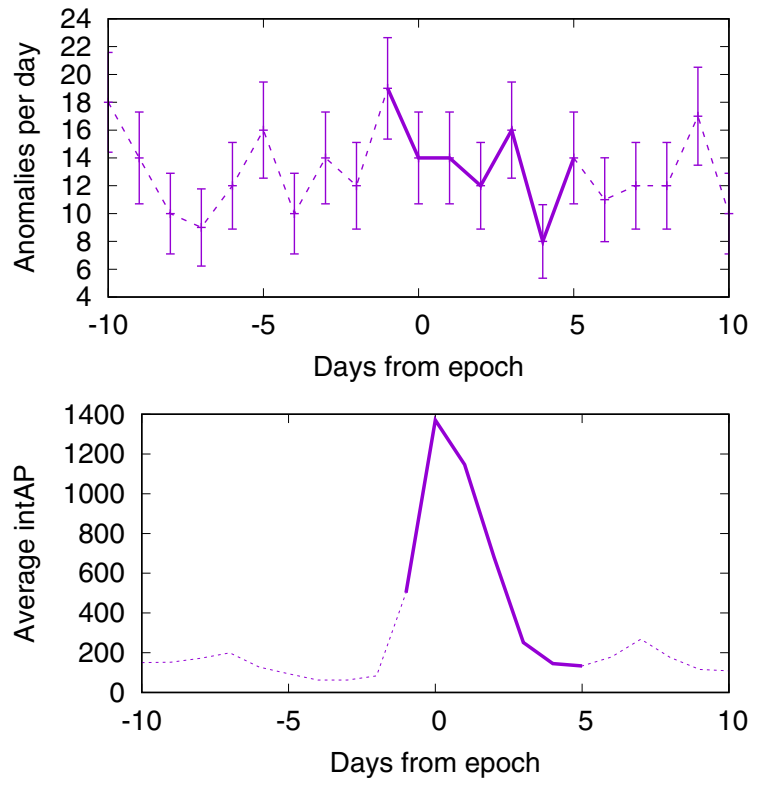

Fig. A.31. Same as Figure A.25 only for series D7.
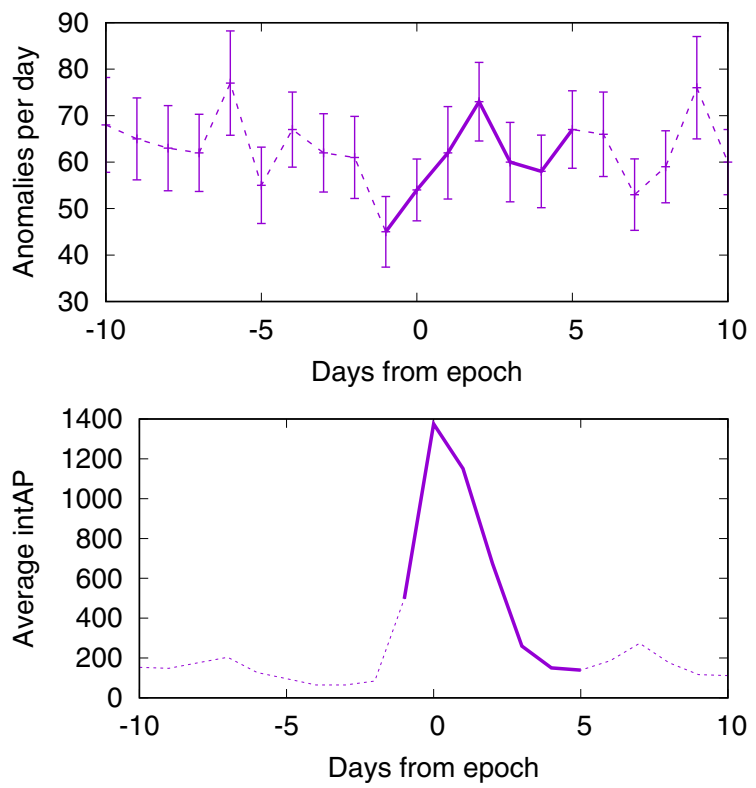

Fig. A.32. Same as Figure A.25 only for series D8. 
M. Švanda et al.: J. Space Weather Space Clim. 2020, 10, 26
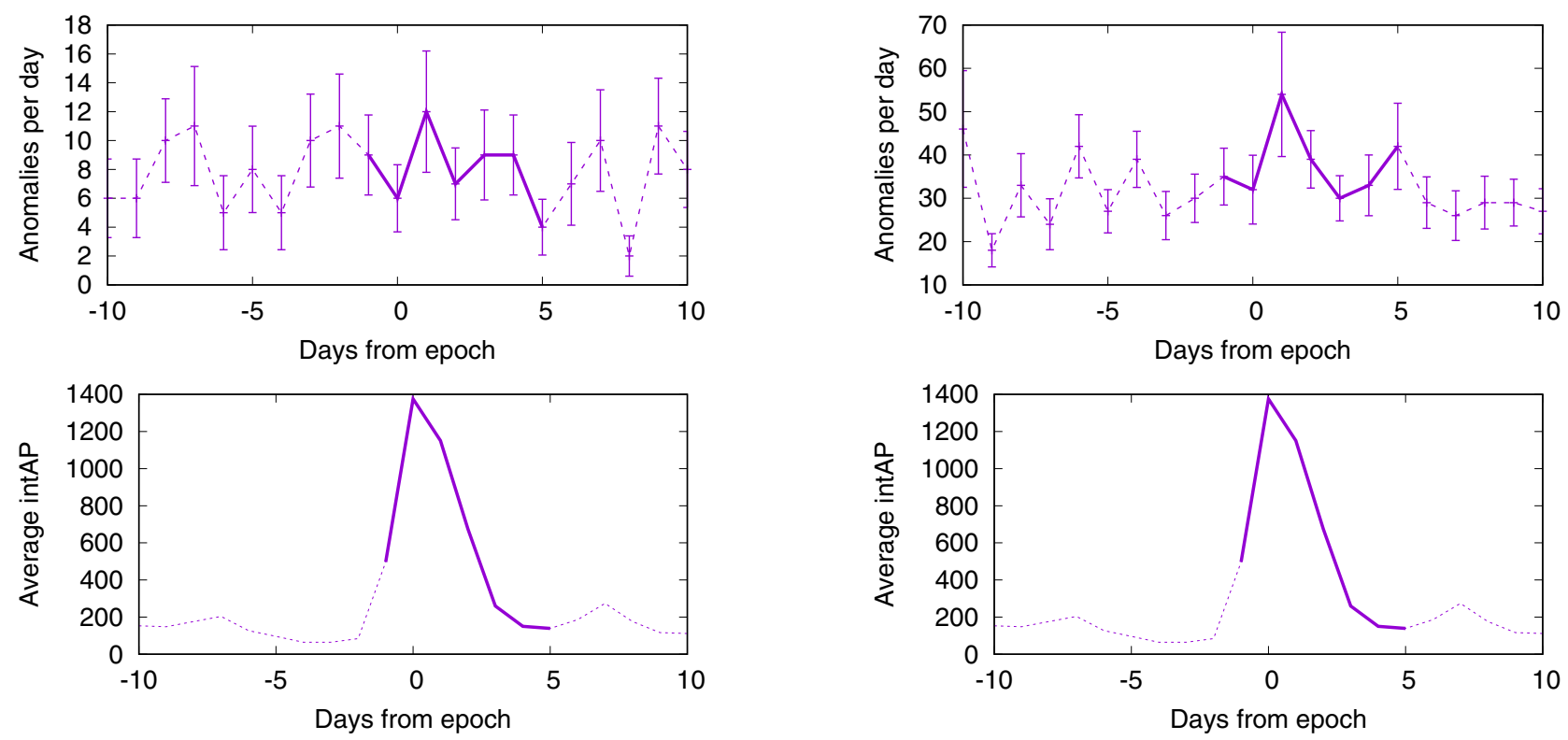

Fig. A.33. Same as Figure A.25 only for series D9.
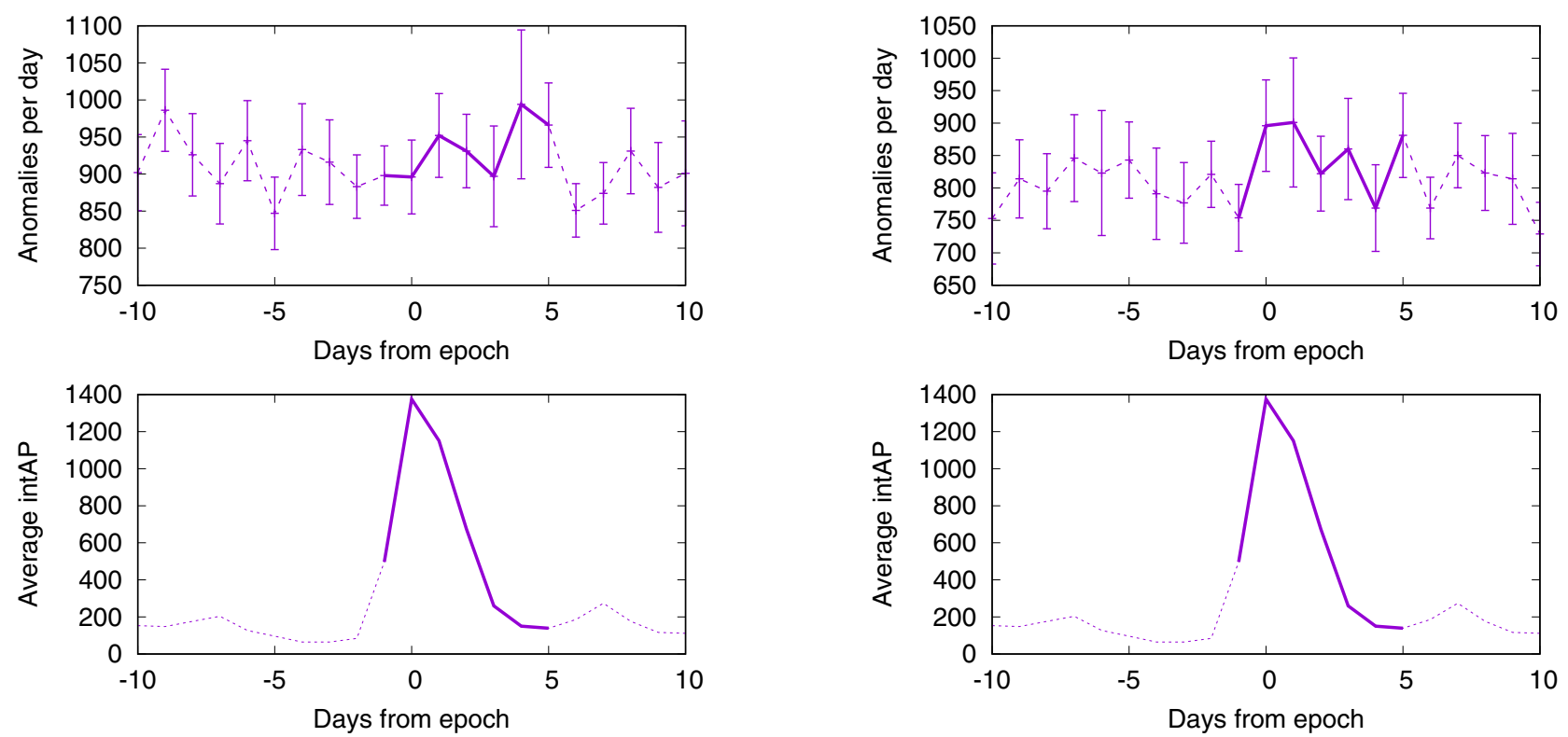

Fig. A.34. Same as Figure A.25 only for series D10.

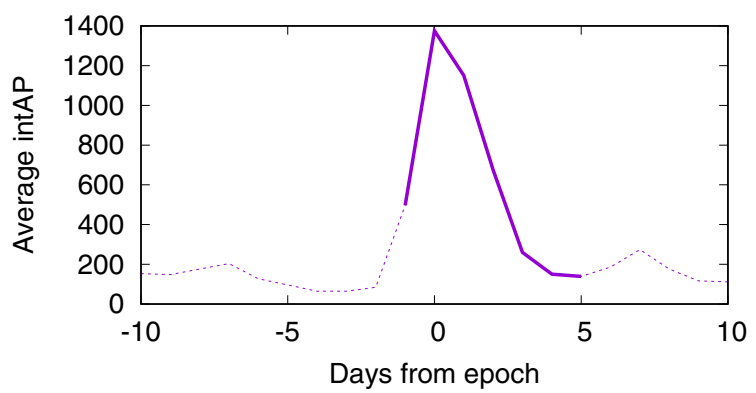

Fig. A.36. Same as Figure A.25 only for series D12. 

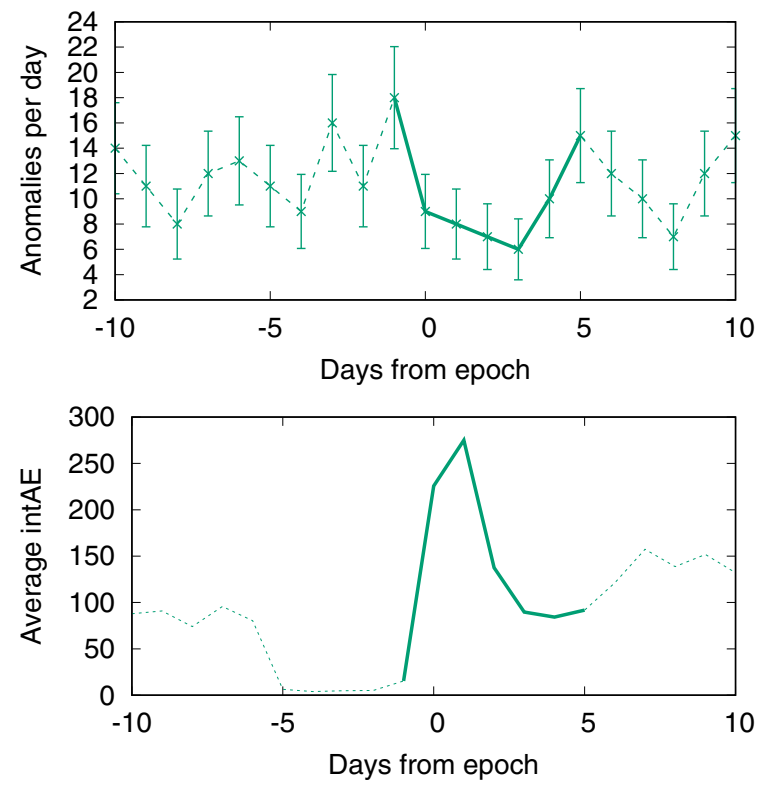

Fig. A.37. Plot of epoch-superposed D1 of increases (or decreases) of the daily number of anomalies as a function of time, considering the IntAE parameter. Solid lines indicate superposed daily rates of anomalies (upper row) or geomagnetic activity IntAE in the reference time series (bottom row) during Days -1 to +5 from the epoch (Day 0 ), whereas dashed lines show the same quantities for the remaining days.
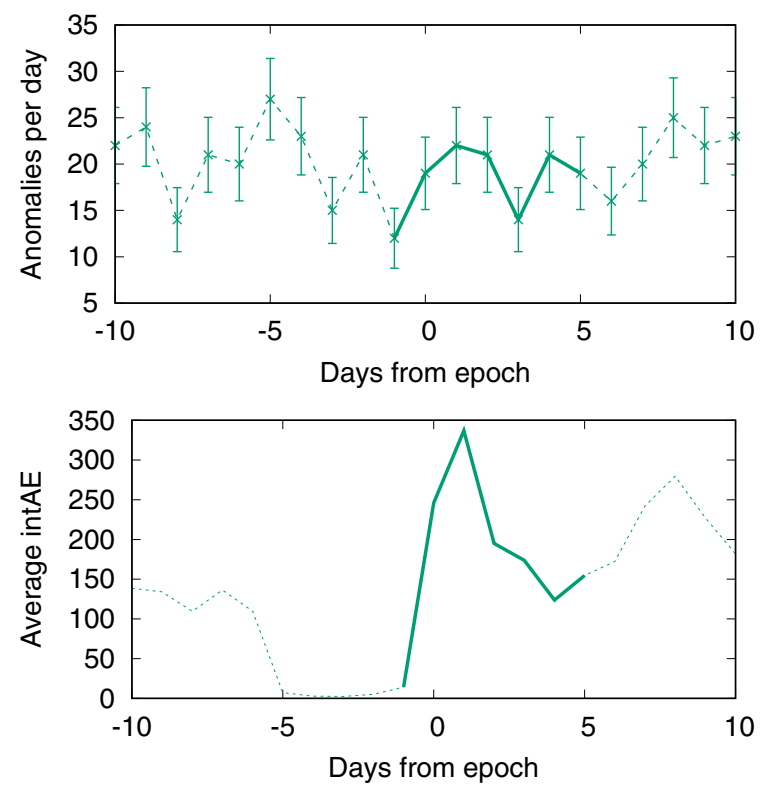

Fig. A.38. Same as Figure A.37 only for series D2.
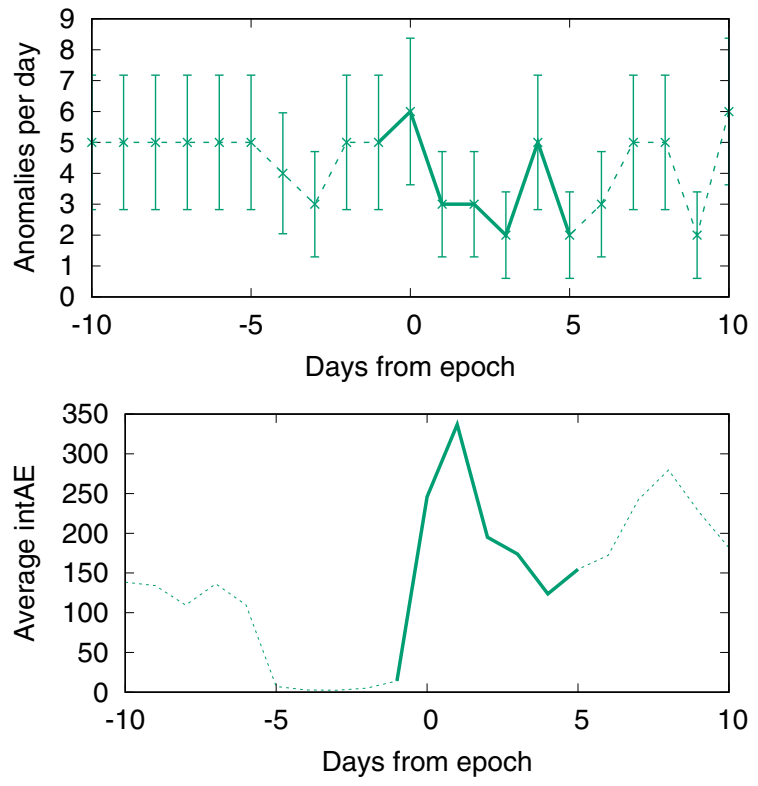

Fig. A.39. Same as Figure A.37 only for series D3.
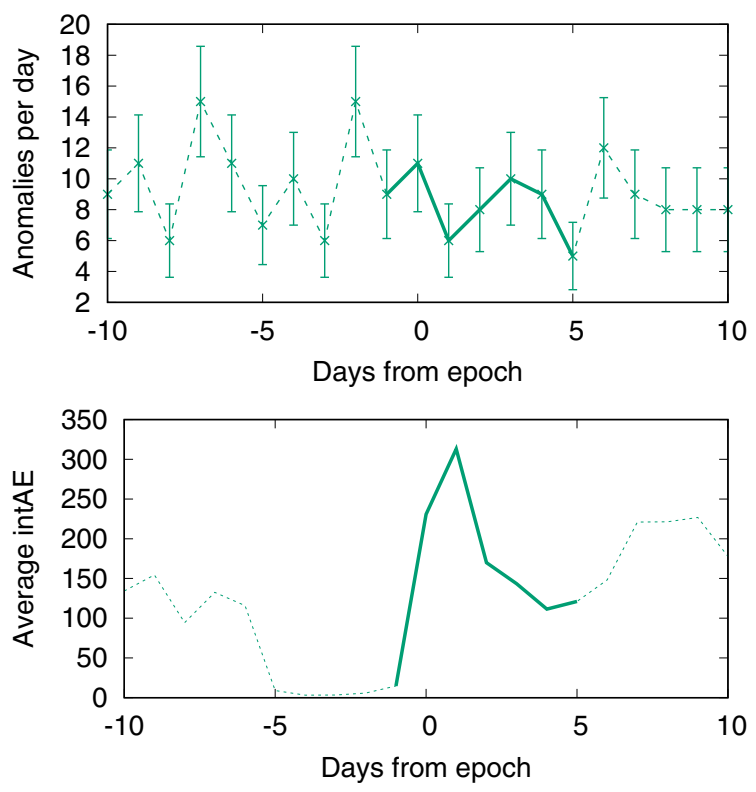

Fig. A.40. Same as Figure A.37 only for series D4. 
M. Švanda et al.: J. Space Weather Space Clim. 2020, 10, 26
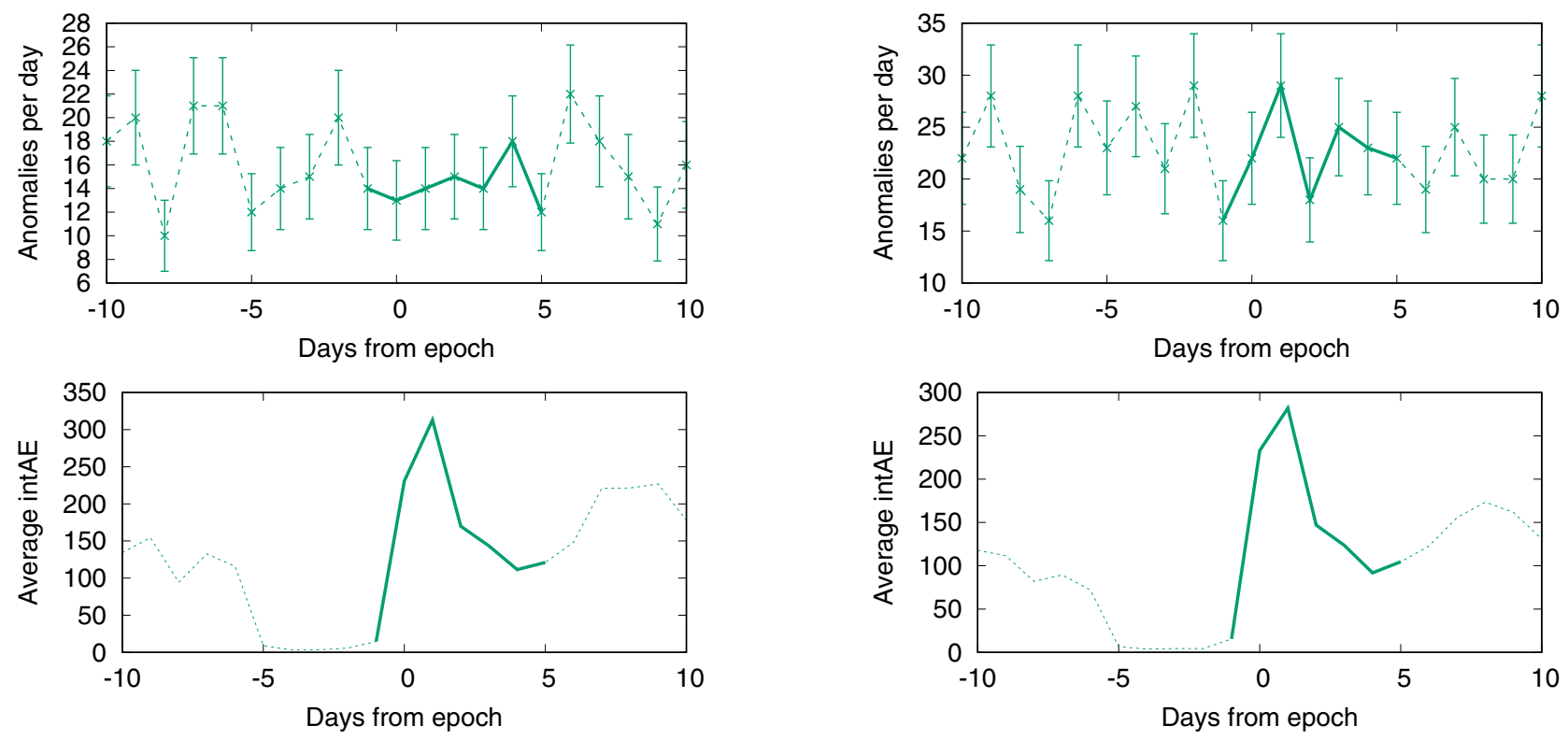

Fig. A.41. Same as Figure A.37 only for series D5.
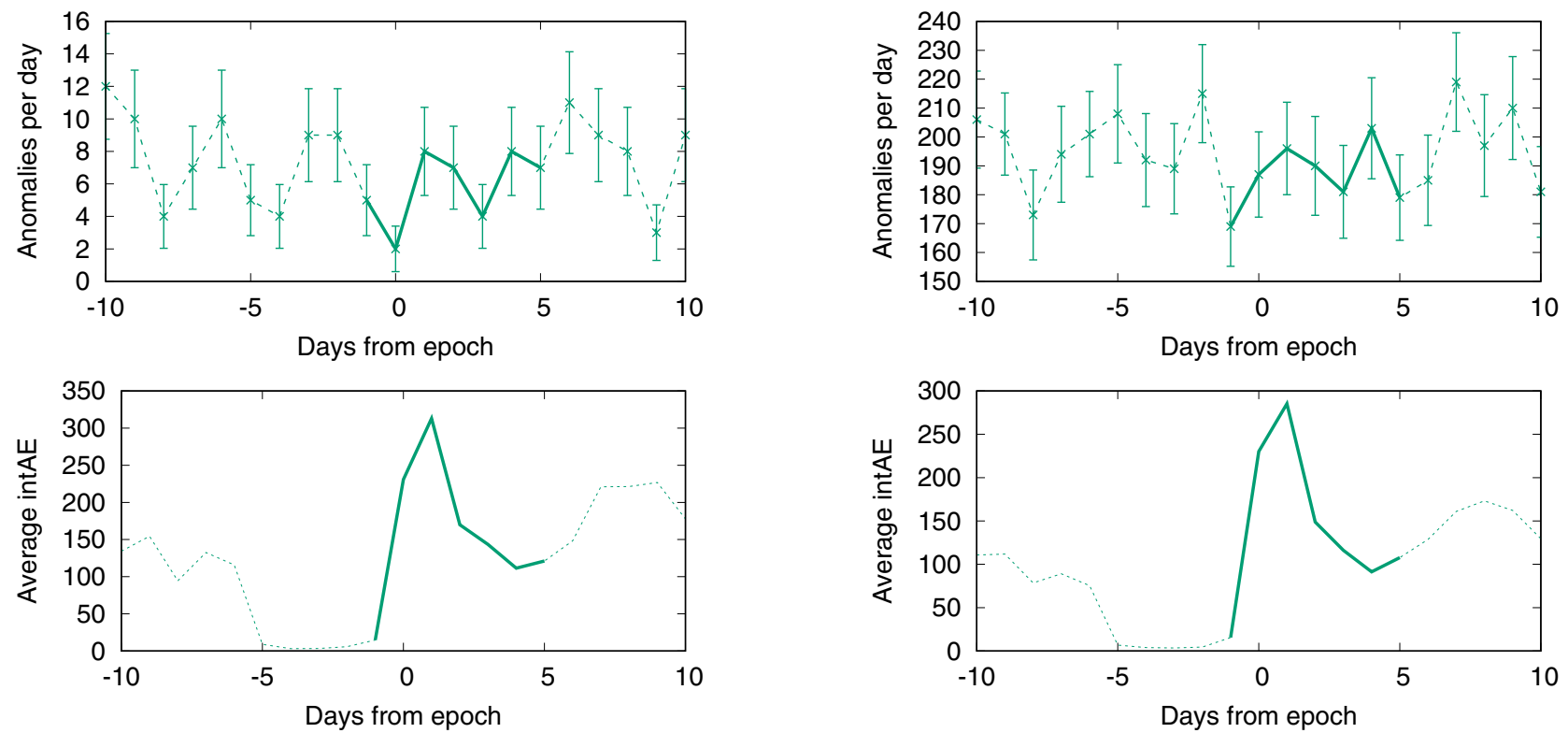

Fig. A.42. Same as Figure A.37 only for series D6.

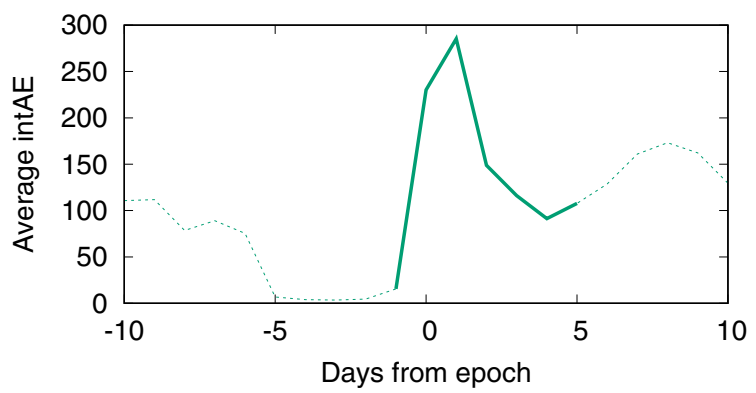

Fig. A.44. Same as Figure A.37 only for series D8. 

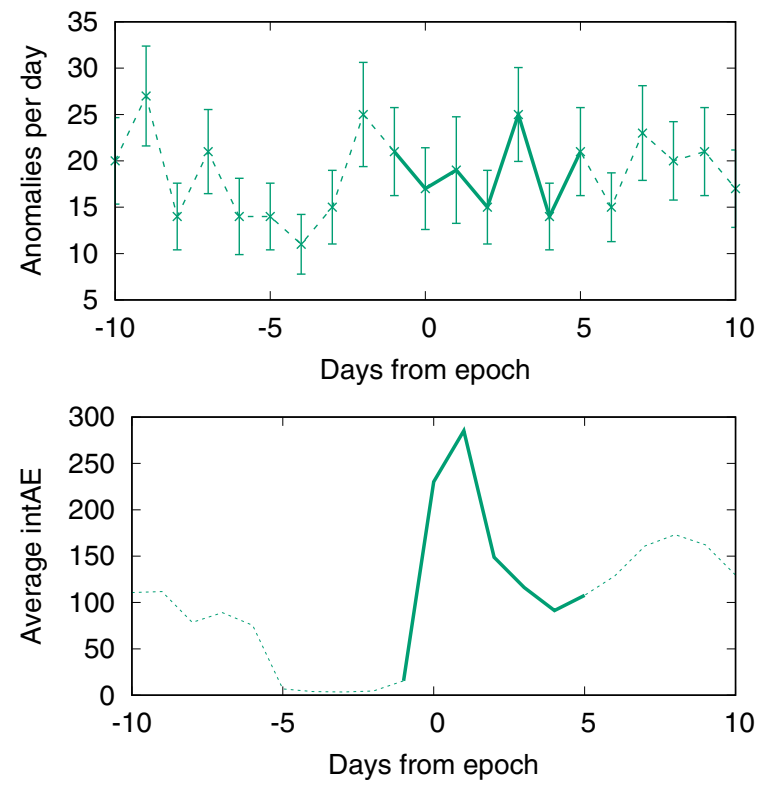

Fig. A.45. Same as Figure A.37 only for series D9.
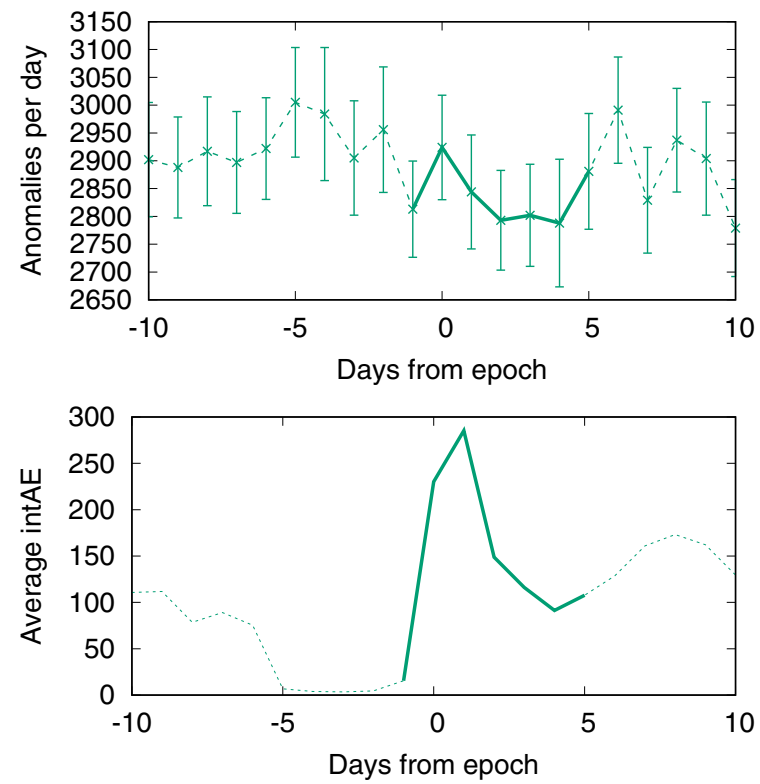

Fig. A.46. Same as Figure A.37 only for series D10.
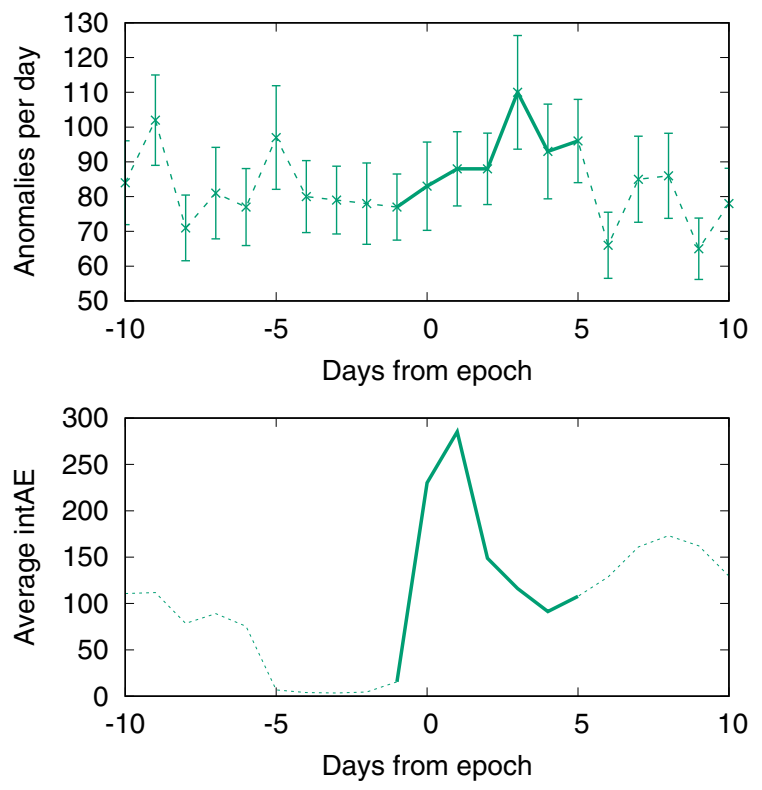

Fig. A.47. Same as Figure A.37 only for series D11.
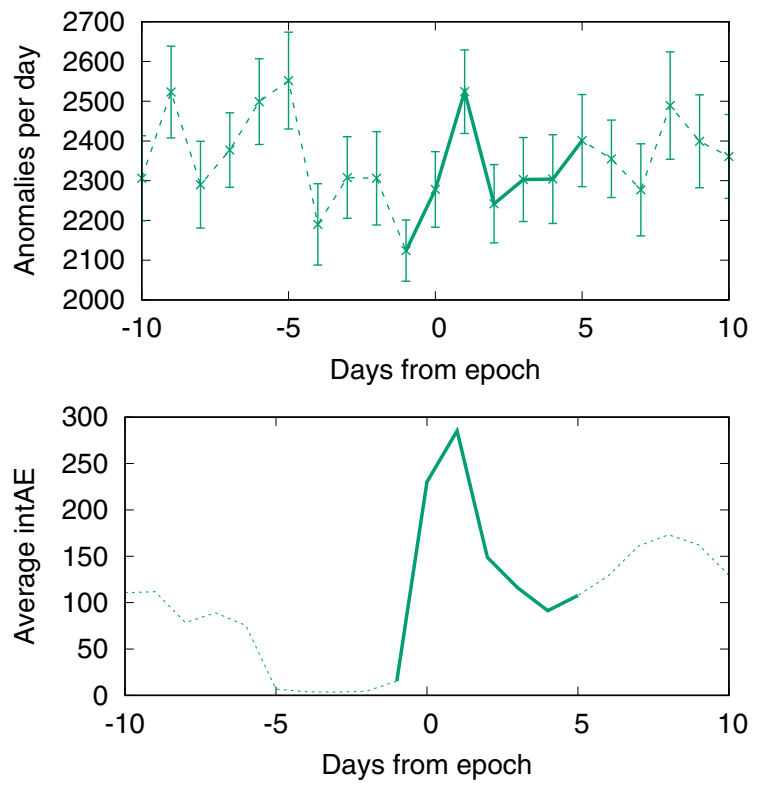

Fig. A.48. Same as Figure A.37 only for series D12.

Cite this article as: Švanda M, Mourenas D, Žertová K \& Výbošt'oková T 2020. Immediate and delayed responses of power lines and transformers in the Czech electric power grid to geomagnetic storms. J. Space Weather Space Clim. 10, 26. 